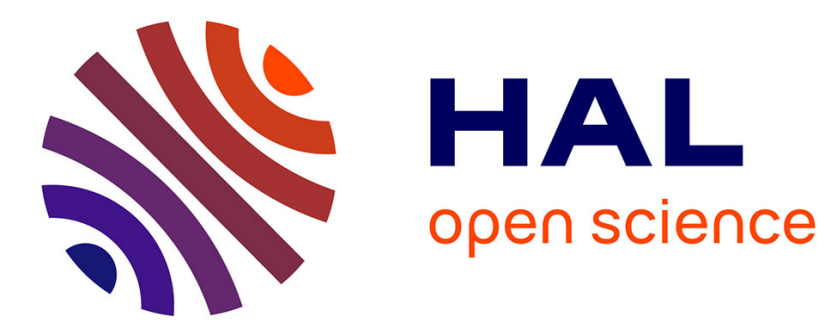

\title{
Stability of stationary viscous incompressible flow around a rigid body performing a translation
}

\author{
Paul Deuring
}

\section{To cite this version:}

Paul Deuring. Stability of stationary viscous incompressible flow around a rigid body performing a translation. 2017. hal-01534693

\section{HAL Id: hal-01534693 \\ https://hal.science/hal-01534693}

Preprint submitted on 8 Jun 2017

HAL is a multi-disciplinary open access archive for the deposit and dissemination of scientific research documents, whether they are published or not. The documents may come from teaching and research institutions in France or abroad, or from public or private research centers.
L'archive ouverte pluridisciplinaire HAL, est destinée au dépôt et à la diffusion de documents scientifiques de niveau recherche, publiés ou non, émanant des établissements d'enseignement et de recherche français ou étrangers, des laboratoires publics ou privés. 


\title{
Stability of stationary viscous incompressible flow around a rigid body performing a translation.
}

\author{
Paul Deuring \\ Université du Littoral Côte d'Opale, Laboratoire de mathématiques pures et appliquées \\ Joseph Liouville, F-62228 Calais, France.
}

\begin{abstract}
AMS subject classifications. 35Q30, 65N30, 76D05.

Key words. Stability, incompressible Navier-Stokes system, Oseen term.
\end{abstract}

\section{Introduction}

Consider a rigid body moving steadily and without rotation in an incompressible viscous fluid. Suppose the fluid flow is steady, too. With respect to a frame in which the body is at rest, such a flow is usually described by the stationary Navier-Stokes system. After normalization, this system takes the form

$$
-\Delta V+\tau(V \cdot \nabla) V+\nabla \Pi=F, \quad \operatorname{div} V=0 \quad \text { in } \bar{\Omega}^{c}:=\mathbb{R}^{3} \backslash \bar{\Omega},
$$

where $\Omega \subset \mathbb{R}^{3}$ is supposed to be open, bounded, with $C^{2}$-boundary and with $\bar{\Omega}^{c}$ connected. In order to obtain a well-posed problem, we require a boundary condition on $\partial \Omega$ and at infinity,

$$
V \mid \partial \Omega=0, \quad V(x) \rightarrow(1,0,0) \text { for }|x| \rightarrow \infty .
$$

The domain $\Omega$ represents the rigid body, the vector field $V$ stands for the unknown velocity field of the fluid, and the scalar function $\Pi$ for the unknown pressure field. The vector $(-1,0,0)$ corresponds to the velocity of the rigid body, as seen from an observer at rest, for whom the fluid velocity tends to zero far from the moving object. Of course, for an observer moving with the rigid body, the velocity of the fluid near infinity is $(1,0,0)$, and thus nonvanishing. Besides this velocity, the given quantities are the volume force $F$, a vector-valued function, and the Reynolds number $\tau \in(0, \infty)$, which is a coefficient describing the character of the flow.

From a mathematical point of view, it is inconvenient to work with nonzero boundary conditions at infinity as in (1.2). Therefore we introduce the new velocity field $U:=$ $V-(1,0,0)$. This field verifies the Navier-Stokes system with the Oseen term $\tau \partial_{1} U$,

$$
-\Delta U+\tau \partial_{1} U+\tau(U \cdot \nabla) U+\nabla \Pi=F, \quad \operatorname{div} U=0 \quad \text { in } \bar{\Omega}^{c},
$$

and is subject to the boundary conditions

$$
U \mid \partial \Omega=(-1,0,0), \quad U(x) \rightarrow 0 \text { for }|x| \rightarrow \infty .
$$

We consider a Leray solution to (1.3), (1.4), that is, a pair of function $(U, \Pi)$ with $U \in$ $L^{6}\left(\bar{\Omega}^{c}\right)^{3} \cap W_{l o c}^{1,1}\left(\bar{\Omega}^{c}\right)^{3}, \nabla U \in L^{2}\left(\bar{\Omega}^{c}\right)^{9}$ and $\Pi \in L_{l o c}^{2}\left(\bar{\Omega}^{c}\right)^{3}$ satisfying (1.3) - in variational 
form - as well as (1.4). The boundary condition at infinity imposed by (1.4) is taken account of by the relations $U \in L^{6}\left(\bar{\Omega}^{c}\right)^{3}, \nabla U \in L^{2}\left(\bar{\Omega}^{c}\right)^{9}$. (We refer to Section 2 for the definition of our function spaces and for other notation.) Leray solutions to (1.3), (1.4) exist under suitable assumptions on $F$ and $\partial \Omega$. More details may be found in $[23$, Theorem VII.2.1, II.5.1]. If $F$ decays sufficiently fast, it may be shown that $U \in L^{p}\left(\bar{\Omega}^{c}\right)^{3}$ for $p \in(2, \infty]$ and $\nabla U \in L^{p}\left(\bar{\Omega}^{c}\right)^{9}$ for $p \in(4 / 3, \infty]$ ([24, Theorem X.4.1]). In the work at hand, it is sufficient to suppose $U \in L^{p}\left(\bar{\Omega}^{c}\right)^{3}$ for $p \in(2, \infty)$ and $\nabla U \in L^{p}\left(\bar{\Omega}^{c}\right)^{9}$ for $p \in\left(4 / 3,3+\epsilon_{0}\right]$, with some $\epsilon_{0}>0$. Then $U \in W^{1,3+\epsilon_{0}}\left(\bar{\Omega}^{c}\right)^{3}$, so $U \in L^{\infty}\left(\bar{\Omega}^{c}\right)^{3}$ by a Sobolev inequality, and also $|U(x)| \rightarrow 0$ for $|x| \rightarrow \infty$, as explained in [12, p. 209]. Therefore the assumptions $U \in L^{p}\left(\bar{\Omega}^{c}\right)^{3}$ for $p \in(2, \infty)$ and $\nabla U \in L^{p}\left(\bar{\Omega}^{c}\right)^{9}$ for $p \in\left(4 / 3,3+\epsilon_{0}\right]$ mean in particular that

$$
\begin{aligned}
& U \in L^{p}\left(\bar{\Omega}^{c}\right)^{3} \text { for } p \in(2, \infty], \quad|U(x)| \rightarrow 0 \text { for }|x| \rightarrow \infty, \\
& \nabla U \in L^{p}\left(\bar{\Omega}^{c}\right)^{9} \text { for } p \in(4 / 3,3] .
\end{aligned}
$$

These are the properties of $U$ we will actually use in what follows. We are interested in conditions which guarantee asymptotic stability of $U$, that is, if $\widetilde{U} \in W_{0}^{1,2}\left(\bar{\Omega}^{c}\right)^{3} \cap H_{2}\left(\bar{\Omega}^{c}\right)$, and if the quantity $\|U-\widetilde{U}\|_{2}+\| \nabla\left(U-\widetilde{U} \|_{2}\right.$ is sufficiently small, then the velocity part $v$ of a solution $(v, \pi)$ of the initial-boundary value problem

$$
\begin{aligned}
& v^{\prime}(t)-\Delta_{x} v(t)+\tau \partial x_{1} v(t)+\tau\left(v(t) \cdot \nabla_{x}\right) v(t)+\nabla_{x} \pi(t)=F, \quad \operatorname{div}_{x} v(t)=0(t>0),(1.5) 4 \\
& v \mid \partial \Omega \times(0, \infty)=(-1,0,0), \quad v(0)=\widetilde{U},
\end{aligned}
$$

should satisfy the relation $\left\|\nabla_{x}(v(t)-\widetilde{U})\right\|_{2} \rightarrow 0$ for $t \rightarrow \infty$. However - and this is usual in this context - we will not consider problem (1.6), (1.7) directly. Instead we work with the equivalent problem

$$
\begin{aligned}
& u^{\prime}(t)-\Delta_{x} u(t)+\tau \partial x_{1} u(t)+\tau\left(U \cdot \nabla_{x}\right) u(t) \\
& +\tau(u(t) \cdot \nabla) U+\tau\left(u(t) \cdot \nabla_{x}\right) u(t)+\nabla_{x} \pi(t)=0, \quad \operatorname{div}_{x} u(t)=0 \quad \text { for } t \in(0, \infty), \\
& v \mid \partial \Omega \times(0, \infty)=0, \quad u(0)=u_{0} .
\end{aligned}
$$

Our aim then is to show stability of the zero solution to (1.8), (1.9), in the sense that

$$
\left\|\nabla_{x} u(t)\right\|_{2} \rightarrow 0(t \rightarrow \infty) \text { if the quantity }\left\|u_{0}\right\|_{2}+\left\|\nabla u_{0}\right\|_{2} \text { is small. }
$$

Of course, the preceding relation will only hold under certain assumptions. The type of conditions we are interested in here relate to the spectrum of a linear operator $\mathcal{L}$, which may be defined as follows. Put

$$
\begin{aligned}
& \mathcal{D}(\mathcal{L}):=H_{2}\left(\bar{\Omega}^{c}\right) \cap W^{2,2}\left(\bar{\Omega}^{c}\right)^{3} \cap W_{0}^{1,2}\left(\bar{\Omega}^{c}\right)^{3}, \\
& \mathcal{B}(W):=-\tau(U \cdot \nabla) W-\tau(W \cdot \nabla) U \quad \text { for } W \in W_{\text {loc }}^{1,1}\left(\bar{\Omega}^{c}\right)^{3} \\
& \mathcal{L}_{0}(W):=\mathcal{P}_{2}\left(\Delta W-\tau \partial_{1} W\right) \quad \text { "Oseen operator"), } \\
& \mathcal{L}(W):=\mathcal{L}_{0}(W)+\mathcal{P}_{2}(\mathcal{B}(W))=\mathcal{P}_{2}\left(\Delta W-\tau \partial_{1} W+\mathcal{B}(W)\right) \text { for } W \in \mathcal{D}(\mathcal{L}) .(1.13) 70
\end{aligned}
$$

Under the assumption $\Omega=\emptyset$ (hence $\bar{\Omega}^{c}=\mathbb{R}^{3}$, "whole space case"), it was proved by J. Neustupa and the present author [12] that the stability relation in (1.10) holds if $\Re \lambda<0$ 
for any eigenvalue $\lambda$ of $\mathcal{L}$, and if $\lambda=0$ is not a generalized eigenvalue of $\mathcal{L}$. The reason why we restricted ourselves to the whole space case was that only then we were able to derive the resolvent estimates necessary for our argument.

In a recent paper [45], J. Neustupa gave a proof of (1.10) under the assumptions that $\lambda=0$ is not a generalized eigenvalue of $\mathcal{L}$, and that there is some $\delta>0$ such that any eigenvalue $\lambda$ of $\mathcal{L}$ satisfies one of the relations

$$
\Re \lambda \leq-\delta \text { or } \tau^{2} \Re \lambda \leq-(\Im \lambda)^{2} .
$$

The second inequality means that $\lambda$ belongs to the essential spectrum of $\mathcal{L}$. In fact, according to Babenko [2], Farwig, Neustupa [16], the essential spectrum $\sigma_{\text {ess }}\left(\mathcal{L}_{0}\right)$ of the Oseen operator $\mathcal{L}_{0}($ see $(1.13))$ is given by $\sigma_{\text {ess }}\left(\mathcal{L}_{0}\right)=\left\{\lambda \in \mathbb{C}: \tau^{2} \Re \lambda \leq-(\Im \lambda)^{2}\right\}$. As explained in $\left[44\right.$, p. 25], the essential spectrum $\sigma_{\text {ess }}(\mathcal{L})$ of $\mathcal{L}$ coincides with that of $\mathcal{L}_{0}$, hence

$$
\sigma_{\text {ess }}(\mathcal{L})=\sigma_{\text {ess }}\left(\mathcal{L}_{0}\right)=\left\{\lambda \in \mathbb{C}: \tau^{2} \Re \lambda \leq-(\Im \lambda)^{2}\right\} .
$$

This means $\sigma_{\text {ess }}(\mathcal{L})$ is a parabolic region in the complex plane touching the imaginary axis from the left at the origin $\lambda=0$.

The conditions in (1.15) are stronger than those required in [12] for the whole space case: stability holds in that latter situation even if contrary to (1.15), there is no $\delta>0$ such that $\Re \lambda \leq-\delta$ for any eigenvalue $\lambda$ not belonging to the essential spectrum of $\mathcal{L}$. Instead, as mentioned above, it is sufficient that $\Re \lambda>0$. Reference [45] leaves open the question whether the result from [12] remains valid in the exterior domain case. It is the aim of the work at hand to give a positive answer to this question (Theorem 2.5).

This is not just a technical improvement. In fact, simple examples involving the linear problem $u^{\prime}(t)-\mathcal{L}(u(t))=0$ indicate that stability cannot be expected to hold if there are eigenvalues of $\mathcal{L}$ with nonnegative real part. (As explained in [45] by a reference to [31] and [46], the nonlinear term in (1.6) does not contribute to stability.) Thus, by allowing eigenvalues with negative real part to pile up near the imaginary axis - including those that do not lie in the essential spectrum -, we clearly deal with a main difficulty, and obtain an optimal result. So it is perhaps not astonishing that a considerable effort is necessary to derive this result.

As a key feature of our proof, the resolvent estimates established in [12] for the whole space case will be generalized to the exterior domain case. (We will comment on these estimates further below.) Once they are available, the functional analytic reasoning leading to (1.10) may be carried through in exactly the same way as in [12]. Our resolvent estimates may serve in other contexts as well, and thus are of independent interest.

The theory developed here, in [45] and in [12], respectively, goes beyond of what may be expected from the theory of abstract differential equations. In fact, according to [46] or [30], [31] for example, stability holds in an abstract situation if the relevant spectrum is located in the part $\Re \lambda \leq-\delta$ of the complex plane, for some $\delta>0$. Such a condition cannot be satisfied here because the essential spectrum of $\mathcal{L}$ touches the imaginary axis from the left, as mentioned above (see (1.16)). But the point is that the work at hand, [12] and [45] make do with weaker assumptions than those in the abstract case, requiring conditions only on eigenvalues, instead of on all of the spectrum. 
The present work, reference [12] and [45] share a common starting point. In fact, all three of them are based on a result by J. Neustupa [44, Section 4] (also see the predecessor papers [41], [42], [43]), which we formulate in Theorem 2.4 below, and which states that the relation in (1.10) holds true if the semigroup generated by $\mathcal{L}$ satisfies a certain estimate on a certain finite-dimensional subspace of $\mathcal{D}(\mathcal{L})$ (inequality $(2.3)$ ). However, this criterion is very technical, should be difficult to verify in concrete situations, and does not connect with the more general theory based on spectral properties of suitable linear operators. This situation motivated the articles [12], [45] as well as the work at hand.

A remark is perhaps in order with respect to the condition that $\lambda=0$ is not a generalized eigenvalue of $\mathcal{L}$. A precise form of this assumption states that the only solution of the boundary value problem

$$
-\Delta V+\tau \partial_{1} V-\mathcal{B}(V)+\nabla \Pi=0, \quad \operatorname{div} V=0 \quad \text { in } \bar{\Omega}^{c}, \quad V \mid \partial \Omega=0,
$$

whose first and second derivatives belong to $L^{2}\left(\bar{\Omega}^{c}\right)$ is the zero function. Note that the velocity part $V$ of a solution to (1.17) cannot be expected to belong to $L^{2}\left(\bar{\Omega}^{c}\right)^{3}$, in view of a similar situation for solutions to the Oseen system ([24, Theorem X.6.4]). Thus, supposing that $\lambda=0$ is not an eigenvalue of $\mathcal{L}$ amounts to not requiring anything at all concerning the spectral properties of the origin of the complex plane. Therefore it should be expected that in the case $\lambda=0$, a somewhat more general notion of eigenvalue is needed in order to guarantee stability.

As indicated above, our proofs are based on resolvent estimates. More precisely, we first estimate the velocity part $V$ of solutions to the Oseen resolvent problem,

$$
-\Delta V+\tau \partial_{1} V+\lambda V+\nabla \Pi=G, \quad \operatorname{div} V=0 \quad \text { in } \bar{\Omega}^{c}, \quad V \mid \partial \Omega=0,
$$

and then the velocity part of solutions to a perturbed version of (1.18), that is,

$$
-\Delta V+\tau \partial_{1} V-\mathcal{B}(V)+\lambda V+\nabla \Pi=G, \quad \operatorname{div} V=0 \quad \text { in } \bar{\Omega}^{c}, \quad V \mid \partial \Omega=0 .
$$

Both in the case of problem (1.18) and (1.19), we provide upper bounds of the velocity depending on $\lambda$ in an explicit way. This is - as usual - the crucial feature and the main difficulty of resolvent estimates. In a paper by Kobayashi, Shibata [32] - the article in literature most closely related in spirit to our work -, this type of estimates for solutions to (1.18) are derived under the assumptions $\Re \lambda \geq 0,|\lambda| \geq C_{0}$ for some $C_{0}>0$ ([32, Theorem 4.4]). Our key estimates of solutions to (1.18) are in a certain sense complementary: we provide upper bounds holding uniformly for $|\lambda| \rightarrow 0$ if $\Re \lambda \geq 0$. In this context, it is important to know that a basic resolvent estimate for the Stokes system does not have an equivalent in the Oseen case. More precisely, it is well known that $\|V\|_{2} \leq C\|G\|_{2} /|\lambda|$ if $V$ is the velocity part of a solution to the Stokes resolvent system $-\Delta V+\lambda V+\nabla \Pi=$ $G, \operatorname{div} V=0$ in $\bar{\Omega}^{c}$, under Dirichlet boundary conditions $V \mid \partial \Omega=0$, where $G \in L^{2}(\bar{\Omega})^{3}$ and $\lambda \in \mathbb{C}$ with $|\arg \lambda| \leq \vartheta$, with $\vartheta$ being some fixed number from $(\pi / 2, \pi)$. Such an estimate does not carry over to the Oseen resolvent problem (1.18) if $\lambda$ is close to zero. This is obvious in the case $\Re \lambda<0$, in view of what is stated above about $\sigma_{\text {ess }}\left(\mathcal{L}_{0}\right)$. But as was shown in [13], this estimate does not hold for the Oseen resolvent even under the assumption $\Re \lambda \geq 0$ if $|\lambda|$ is small. 
Concerning further results in existing literature, we mention that Sazonov [47] announced a stability result as in (1.10), but with $\|u(t)\|_{3}$ in the place of $\|\nabla u(t)\|_{2}$, under assumptions on the spectrum of $\mathcal{L}$ similar to ours. But it seems his proof has a gap; see the detailed discussion of his argument in [44, p. 25], [45, Section 1], and [32, p. 6-7].

There are many articles deriving stability results for the zero solution to (1.8), (1.9) from smallness conditions on $\tau$ or $U$. In this regard we mention J. G. Heywood [27], [28], [29], K. Masuda [38], P. Maremonti [37], G. P. Galdi and S. Rionero [20], G. P. Galdi and M. Padula [21], W. Borchers and T. Miyakawa [3], [4], H. Kozono and T. Ogawa [34], H. Kozono and M. Yamazaki [35], [36], G. P. Galdi, J. G. Heywood and Y. Shibata [22], T. Miyakawa [39] and Y. Shibata [48]. This list is not exhaustive.

\section{Notation. Statement of main results.}

For $\vartheta \in(0, \pi), a \in(0, \infty)$, we put $S_{\vartheta, a}:=\{\lambda \in \mathbb{C} \backslash\{a\}:|\arg (\lambda-a)| \leq \vartheta\}$. If $A \subset \mathbb{R}^{3}$, let $A^{c}$ denote the complement $\mathbb{R}^{3} \backslash A$ of $A$ in $\mathbb{R}^{3}$. The symbol || designates the Euclidean norm in $\mathbb{R}^{n}$, for any $n \in \mathbb{N}$, and also the length $\alpha_{1}+\alpha_{2}+\alpha_{3}$ of a multiindex $\alpha \in \mathbb{N}_{0}^{3}$, as well as the Lebesgue measure of measurable subsets of $\mathbb{R}^{3}$. For $R>0, x \in \mathbb{R}^{3}$, put $B_{R}(x):=\left\{y \in \mathbb{R}^{3}:|x-y|<R\right\}$. If $x=0$, we write $B_{R}$ instead of $B_{R}(0)$. If $A$ is some nonempty set and $\gamma: A \mapsto \mathbb{R}$ a function, we abbreviate $|\gamma|_{\infty}:=\sup \{|\gamma(x)|: x \in A\}$. Let $V: \mathbb{R}^{3} \mapsto \mathbb{C}$ and $W: \mathbb{R}^{3} \mapsto \mathbb{C}$ be measurable functions with $\int_{\mathbb{R}^{3}}|V(x-y)||W(y)| d y<\infty$ for a. e. $x \in \mathbb{R}^{3}$. Then we define $(V * W)(x):=\int_{\mathbb{R}^{3}} V(x-y) W(y) d y$ for a. e. $x \in \mathbb{R}^{3}$. We will use the differential operators $\partial_{i}, \partial^{\beta}$ for $\beta \in \mathbb{N}_{0}^{3}, \nabla, \Delta$ and div with respect to functions having a domain in $\mathbb{R}^{3}$. As concerns function with domain of the form $A \times J$, where $A \subset \mathbb{R}^{3}, J \subset \mathbb{R}$, we use the notation $\partial_{x_{i}}, \partial_{x}^{\beta}, \nabla_{x}, \Delta_{x}, d i v_{x}, \partial_{t}$, with obvious meanings.

Our notation of function spaces does not distinguish between spaces of real-valued and of complex-valued functions because this distinction should be clear from context. In fact, in what follows, spaces of complex-valued functions are relevant only if a resolvent parameter $\lambda \in \mathbb{C}$ is involved. For $p \in[1, \infty], A \subset \mathbb{R}^{3}$ measurable, we write $L^{p}(A)$ for the usual Lebesgue space on $A$ associated with $p$, and \|\|$_{p}$ for the usual norm of this space. Let $A \subset \mathbb{R}^{3}$ be open, $p \in[1, \infty)$ and $m \in \mathbb{N}$. Then the symbol $W^{m, p}(A)$ stands for the usual Sobolev of order $m$ and exponent $p$. The standard norm of this space is designated by \|\|$_{m, p}$. The space $W_{0}^{m, p}(A)$ is defined as the closure of $C_{0}^{\infty}(A)$ with respect to the norm of $W^{m, p}(A)$, and is equipped with this norm. For any open set $B \subset \mathbb{R}^{3}$, and for $p$ and $m$ as before, the spaces $L_{l o c}^{p}(B), W_{l o c}^{m, p}(B)$ are to contain the functions $v$ from $B$ into $\mathbb{R}$ or $\mathbb{C}$ satisfying the relations $v \mid A \in L^{p}(A)$ and $v \mid A \in W^{m, p}(A)$, respectively, for any open and bounded set $A \subset \mathbb{R}^{3}$ with $\bar{A} \subset B$. If $A \subset \mathbb{R}^{3}$ is an open set with bounded $C^{2}$-boundary $\partial A$, then the Sobolev space $W^{r, p}(\partial A)$ with $p \in(1, \infty), r \in(1,2)$ is to be defined as in $[19$, Section 6.8.6].

Let $n \in \mathbb{N}, A$ a non-empty set and $\mathcal{V}$ a vector space consisting of functions from $A$ into $\mathbb{R}$ or $\mathbb{C}$. Suppose a norm denoted by \|\| has been introduced on this space. Then we set $\left\|\left(f_{1}, \ldots, f_{n}\right)\right\|^{(n)}:=\left(\sum_{j=1}^{n}\left\|f_{j}\right\|^{2}\right)^{1 / 2}$ for $\left(f_{1}, \ldots, f_{n}\right) \in \mathcal{V}^{n}$. The mapping \|\|$^{(n)}$ is a norm on $\mathcal{V}^{n}$, and we equip $\mathcal{V}^{n}$ with this norm. However, we will write \|\| for this norm too, instead of \|\|$^{(n)}$. 
If $A \subset \mathbb{R}^{3}$ is open and $p \in(1, \infty)$, define $H_{p}(A)$ as the closure of the set $\left\{\phi \in C_{0}^{\infty}(A)^{3}\right.$ : $\operatorname{div} \phi=0\}$ with respect to the norm \|\|$_{p}$ of $L^{p}(A)^{3}$. Let $A \subset \mathbb{R}^{3}, p \in(1, \infty), n \in \mathbb{N}$. Then we write $W_{0}^{-1, p}(A)^{n}$ for the space dual to $W_{0}^{1, p^{\prime}}(A)^{n}$. The norm \|\|$_{-1, p}$ of $W_{0}^{-1, p}(A)^{n}$ is to be understood as the operator norm of this space, that is, $\|\mathfrak{F}\|_{-1, p}:=\sup \left\{|F(V)| /\|V\|_{1, p^{\prime}}\right.$ : $\left.V \in W_{0}^{1, p^{\prime}}(A)^{n}, V \neq 0\right\}$ for any linear bounded functional $\mathfrak{F}$ on $W_{0}^{1, p^{\prime}}(A)^{n}$. For any Hilbert space $H$ and any $T \in(0, \infty]$, the spaces $L^{2}(0, T, H)$ and $W^{1,2}(0, T, H)$ are to be defined in the usual way; see [50, Section IV.1.2] for example. The spaces $L_{l o c}^{2}([0, \infty), H)$ and $W_{l o c}^{1,2}([0, \infty), H)$ are to contain those functions $v:(0, \infty) \mapsto H$ satisfying the relation $v \mid(0, T) \in L^{2}(0, T, H)$ and $v \mid(0, T) \in W^{1,2}(0, T, H)$, respectively, for any $T \in(0, \infty)$.

The set $\Omega$ and the parameter $\tau \in(0, \infty)$ introduced in Section 1 will be kept fixed throughout. Recall that $\Omega$ is supposed to be open and bounded, with $C^{2}$-boundary and with $\bar{\Omega}^{c}$ connected. Put $K_{\tau}:=\left\{\lambda \in \mathbb{C}: \Re \lambda \geq 0,|\lambda| \leq(\tau / 2)^{2}\right\}$. For $R \in(0, \infty)$ with $\bar{\Omega} \subset B_{R}$, we put $\Omega_{R}:=B_{R} \backslash \bar{\Omega}$. It will be convenient to fix a real $S \in(0, \infty)$ with $\bar{\Omega} \subset B_{S}$. The symbol $\mathfrak{I}$ stands for the identity operator on $H_{2}\left(\bar{\Omega}^{c}\right)$. By $\mathcal{D}_{0, \sigma}^{1,2}\left(\bar{\Omega}^{c}\right)$, we denote the set of all functions $V \in W_{l o c}^{1,1}\left(\bar{\Omega}^{c}\right)^{3}$ with $\nabla V \in L^{2}(\bar{\Omega})^{9}$ and such that there is a sequence $\left(\phi_{n}\right)$ in $C_{0}^{\infty}\left(\bar{\Omega}^{c}\right)^{3}$ with $\operatorname{div} \phi_{n}=0$ for $n \in \mathbb{N}$ and $\left\|\nabla V-\nabla \phi_{n}\right\|_{2} \rightarrow 0$. We define

$$
\mathcal{D}_{0}^{1,2}\left(\bar{\Omega}^{c}\right):=\left\{V \in W_{l o c}^{1,2}\left(\bar{\Omega}^{c}\right): \nabla V \in L^{2}\left(\bar{\Omega}^{c}\right)^{9}, V \in L^{6}(V) \text { and } V \mid \partial \Omega=0\right\} .
$$

The mapping $V \mapsto\|\nabla V\|_{2}, V \in \mathcal{D}_{0}^{1,2}\left(\bar{\Omega}^{c}\right)$, is a norm (Theorem 3.5); we equip the space $\mathcal{D}_{0}^{1,2}\left(\bar{\Omega}^{c}\right)$ with this norm. It should be remarked that the mapping $V \mapsto\|\nabla V\|_{2}$ is not a norm on $\mathcal{D}_{0}^{1,2}\left(\mathbb{R}^{3}\right)$, contrary to what is written in [12, p. 205], if that latter space is defined as the closure of $C_{0}^{\infty}\left(\mathbb{R}^{3}\right)$ with respect to the mapping $V \mapsto\|\nabla V\|_{2}$. Here this space will not be used. The space dual to $\mathcal{D}_{0}^{1,2}\left(\bar{\Omega}^{c}\right)^{3}$ is denoted by $\mathcal{D}_{0}^{-1,2}\left(\bar{\Omega}^{c}\right)^{3}$. It is equipped with its natural norm denoted by \|\|$_{-1,2}$ and defined by

$$
\|\mathfrak{F}\|_{-1,2}:=\sup \left\{|F(\gamma)| /\|\nabla \gamma\|_{2}: \gamma \in \mathcal{D}_{0}^{1,2}\left(\bar{\Omega}^{c}\right)^{3}, \gamma \neq 0\right\},
$$

for any linear and bounded functional $\mathfrak{F}$ from $\mathcal{D}_{0}^{1,2}\left(\bar{\Omega}^{c}\right)^{3}$ into $\mathbb{R}$ or $\mathbb{C}$. We refer to Theorem 3.6 for more properties of this space. In this theorem, it is explained in which sense functions with domain $\bar{\Omega}^{c}$ may belong to $\mathcal{D}_{0}^{-1,2}\left(\bar{\Omega}^{c}\right)^{3}$. For functions $V \in \mathcal{D}_{0}^{-1,2}\left(\bar{\Omega}^{c}\right)^{3} \cap$ $L^{2}\left(\bar{\Omega}^{c}\right)^{3}$, we use the notation $\|V\|_{*}:=\|V\|_{-1,2}+\|V\|_{2}$. If $p \in(1, \infty), n \in\{1,3\}$ and $G \in W_{l o c}^{1,1}\left(\mathbb{R}^{3}\right)^{n}$, we define

$$
\|G\|_{-1, p, \mathbb{R}^{3}}:=\sup \left\{\int_{\mathbb{R}^{3}} G \cdot \gamma d x /\|\nabla \gamma\|_{p^{\prime}}: \gamma \in C_{0}^{\infty}\left(\mathbb{R}^{3}\right)^{n}, \gamma \neq 0\right\} .
$$

We further set $\widetilde{\mathcal{D}}_{0}^{-1, p}\left(\mathbb{R}^{3}\right)^{n}:=\left\{G \in W_{l o c}^{1,1}\left(\mathbb{R}^{3}\right)^{n}:\|G\|_{-1, p, \mathbb{R}^{3}}<\infty\right\}$. The preceding two notation should be considered as abbreviations. We will not use any functional analytic properties of either the mapping \|\|$_{-1, p, \mathbb{R}^{3}}$ or the space $\widetilde{\mathcal{D}}_{0}^{-1, p}\left(\mathbb{R}^{3}\right)^{n}$. For convenience, we

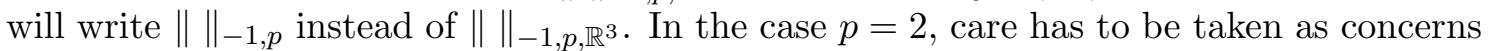
the quantity $\|\phi\|_{-1, p}$ if $\phi \in C_{0}^{\infty}\left(\bar{\Omega}^{c}\right)^{3}$. In fact, for any open set $A \subset \mathbb{R}^{3}$, we consider functions from $C_{0}^{\infty}(A)$ as functions with domain $\mathbb{R}^{3}$ (but, of course, with compact support in $A)$. So, if $\phi \in C_{0}^{\infty}\left(\bar{\Omega}^{c}\right)^{3}$, we have to distinguish between $\|\phi\|_{-1,2}$ and $\left\|\phi \mid \bar{\Omega}^{c}\right\|_{-1,2}$, with the first expression denoting $\|\phi\|_{-1,2, \mathbb{R}^{3}}$, and the second involving the norm \|\|$_{-1,2}$ of $\mathcal{D}_{0}^{-1,2}\left(\bar{\Omega}^{c}\right)^{3}$; see Theorem 3.6 and Lemma 3.4 in this respect. 
The symbol $C$ is to denote numerical constants, and $C\left(\gamma_{1}, \ldots, \gamma_{n}\right)$ constants depending exclusively on $\gamma_{1}, \ldots, \gamma_{n} \in(0, \infty)$, for some $n \in \mathbb{N}$. However, such precise indications on how a constant depends on parameters will be given only at some places. In order to avoid that our presentation becomes too unwieldy, most of the time we take the point of view that the dependence of our constants should be clear from context, and we only indicate some key parameters. In that situation the symbol $\mathfrak{C}$ or $\mathfrak{C}\left(\gamma_{1}, \ldots, \gamma_{n}\right)$ is used to denote generic constants. Typically they depend on $\tau$, on $\Omega$, in particular on the parameter $S$ introduced above, on $\|U\|_{p}$ for certain $p \in(2, \infty]$, and on $\|\nabla U\|_{p}$ for certain $p \in(4 / 3,3]$, where $U$ is the function from (1.5).

We introduce two fundamental solutions. Put $N(x):=(4 \pi|x|)^{-1}$ for $x \in \mathbb{R}^{3} \backslash\{0\}$ (fundamental solution of the Poisson equation), and $E^{(\lambda)}(x):=(4 \pi|x|)^{-1} e^{-\left(\lambda+(\tau / 2)^{2}\right)^{1 / 2}|x|+\tau x_{1} / 2}$ for $x \in \mathbb{R}^{3} \backslash\{0\}, \lambda \in \mathbb{C}$ with $\Re \lambda \geq 0$ (fundamental solution of the scalar Oseen equation $-\Delta V+\tau \partial_{1} V=G$ if $\lambda=0$, and of the associated resolvent equation if $\lambda \neq 0$ ).

We recall that the space $\mathcal{D}(\mathcal{L})$ was introduced in $(1.11)$, and the operators $\mathcal{B}$ and $\mathcal{L}$ in (1.12) and (1.14), respectively. As also mentioned in Section 1, the resolvent set of $\mathcal{L}$ is denoted by $\sigma(\mathcal{L})$. In the ensuing Theorem 2.1, we introduce the Helmholtz projection on $L^{p}(\bar{\Omega})^{3}$, denoted by $\mathcal{P}_{p}$, and the operator $\mathcal{G}_{p}$ corresponding to the complement of $H_{p}\left(\bar{\Omega}^{c}\right)$ in $L^{p}\left(\bar{\Omega}^{c}\right)^{3}$. Theorem 2.1 states those properties of $\mathcal{P}_{p}$ and $\mathcal{G}_{p}$ we take from literature. Some additional facts, in principle well known, too, are presented in Corollary 3.7. Following Theorem 2.1, we introduce the operator $\mathfrak{B}_{\text {sym }}$ and $\mathfrak{K}$ as well as the space $H_{2}^{\prime}$, which come up in Theorem 2.2 and 2.4, and then again in Section 8 (Theorem 8.2, Corollary 8.5). The Helmholtz decomposition of $L^{p}\left(\mathbb{R}^{3}\right)^{3}$, involving operators $P_{p}$ and $G_{p}$, is introduced in Theorem 3.12. The notion of "essential spectrum" mentioned in Section 1, will not be needed in what follows. Still we indicate that in Section 1, the essential spectrum of the operator $\mathcal{L}$ is understood to consist of those $\lambda \in \mathbb{C}$ for which $\lambda \mathfrak{I}-\mathcal{L}$ is not semi-Fredholm ([44, p. 29]).

$\langle$ theoremT2.10〉 Theorem 2.1 ([24, Section III.1, in particular p. 149-152] $)$ Let $\mathcal{P}_{2}: L^{2}\left(\bar{\Omega}^{c}\right)^{3} \mapsto$ $H_{2}\left(\bar{\Omega}^{c}\right)$ denote the usual projection operator onto the closed subspace $H_{2}(\bar{\Omega})$ of $L^{2}\left(\bar{\Omega}^{c}\right)^{3}$. Let $p \in(1, \infty)$. Then there are linear operators $\mathcal{P}_{p}: L^{p}\left(\bar{\Omega}^{c}\right)^{3} \mapsto H_{p}\left(\bar{\Omega}^{c}\right), \mathcal{G}_{p}: L^{p}\left(\bar{\Omega}^{c}\right)^{3} \mapsto$ $W_{\text {loc }}^{1, p}\left(\bar{\Omega}^{c}\right)$ such that $\mathcal{P}_{p}^{2}=\mathcal{P}_{p}, V=\mathcal{P}_{p}(V)+\mathcal{G}_{p}(V)$,

$$
\left\|\mathcal{P}_{p}(V)\right\|_{p}+\|\nabla \mathcal{G}(V)\|_{p} \leq \mathfrak{C}(p)\|V\|_{p},
$$

in particular $\nabla \mathcal{G}_{p}(V) \in L^{p}\left(\bar{\Omega}^{c}\right)^{3}$ and $\mathcal{P}_{p}\left(\nabla \mathcal{G}_{p}(V)\right)=0$, for $V \in L^{p}\left(\bar{\Omega}^{c}\right)^{3}$, and such that $\mathcal{P}_{p}(V)=\mathcal{P}_{2}(V)$ for $V \in C_{0}^{\infty}\left(\bar{\Omega}^{c}\right)^{3}$.

Proof: See [24, Section III.1, in particular p. 149-152] or [18].

Define

$$
\mathcal{B}_{\text {sym }}(W):=\mathcal{P}_{2}\left(-\tau \sum_{j=1}^{3}\left(W_{j}\left[\partial_{k} U_{j}+\partial_{j} U_{k}\right] / 2\right)_{1 \leq k \leq 3}, \quad \mathcal{K}(W):=\mathcal{P}_{2}(\Delta W)\right.
$$

for $W \in \mathcal{D}(\mathcal{L})$. Note that $\mathcal{K}$ is the usual Stokes operator in $L^{2}$. Next we present the results from [44] entering into our theory. We start with a theorem that serves to introduce a key finite-dimensional subspace of $\mathcal{D}(\mathcal{L})$. 
Theorem $2.2\left(\left[44\right.\right.$, Lemma 3]) Fix some $\kappa \in[0,1]$. Then the operator $\mathcal{K}+(1+\kappa) \mathcal{B}_{\text {sym }}$ : $\mathcal{D}(\mathcal{L}) \mapsto H_{2}\left(\bar{\Omega}^{c}\right)$ has a finite number of eigenvalues in $\mathbb{C} \backslash(-\infty, 0]$, all of which are positive and of finite multiplicity.

Observe that each eigenfunction of $\mathcal{K}+(1+\kappa) \mathcal{B}_{\text {sym }}$ belongs to $\mathcal{D}(\mathcal{L})$, in view of the domain of $\mathcal{K}+(1+\kappa) \mathcal{B}_{\text {sym }}$. Denote by $H_{2}^{\prime}$ the subspace of $\mathcal{D}(\mathcal{L})$ generated by the eigenfunctions of $\mathcal{K}+(1+\kappa) \mathcal{B}_{\text {sym }}$ associated with positive eigenvalues of this operator. According to Theorem 2.2, this space $H_{2}^{\prime}$ is finite dimensional.

Theorem 2.3 The operator $\mathcal{L}: \mathcal{D}(\mathcal{L}) \mapsto H_{2}\left(\bar{\Omega}^{c}\right)$ generates an analytic semigroup on $H_{2}(\bar{\Omega})$.

Proof: See [44, p. 32]. Alternatively, this theorem follows from [26, Theorem I.3.4], Lemma 8.5 and Corollary 8.6.

The operator given at time $t \in(0, \infty)$ by the analytic semigroup generated by $\mathcal{L}$ will be denoted by $e^{\mathcal{L} t}$. The theorem from [44] mentioned in Section 1 as being the starting point of our stability theory may now be stated as follows.

$\langle$ theoremT2.40〉

Theorem 2.4 ([44, Theorem 1 and p. 42]) Let $R \in(0, \infty)$ be so large that $\bar{\Omega} \subset B_{R}$ and such that $\left\|U \mid B_{R}^{c}\right\|_{3} \leq C_{0}$ (see (1.5)) for some constant $C_{0}$ independent of $U$. Suppose there is a function $\varphi \in L^{1}(0, \infty) \cap L^{2}(0, \infty)$ such that

$$
\left\|\nabla e^{\mathcal{L} t} \phi \mid \Omega_{R}\right\|_{2} \leq \varphi(t)\|\phi\|_{2} \quad \text { for } t \in(0, \infty), \phi \in H_{2}^{\prime} .
$$

Then there is some number $\delta>0$ such that for any $u_{0} \in H_{2}\left(\bar{\Omega}^{c}\right) \cap \mathcal{D}_{0, \sigma}^{1,2}\left(\bar{\Omega}^{c}\right)$ with $\left\|u_{0}\right\|_{1,2} \leq$ $\delta$, there is a unique function $u \in L_{\text {loc }}^{2}([0, \infty), \mathcal{D}(\mathcal{L})) \cap W_{\text {loc }}^{1,2}\left([0, \infty), H_{2}\left(\bar{\Omega}^{c}\right)\right)$ which solves (1.8), (1.9) in the sense that

$$
u^{\prime}(t)=\mathcal{L}(u(t))+\mathcal{P}_{2}\left[-\tau\left(u(t) \cdot \nabla_{x}\right) u(t)\right], \quad u(0)=u_{0} .
$$

This function satisfies (1.10), that is, $\|\nabla u(t)\|_{2} \rightarrow 0$ for $t \rightarrow \infty$.

As indicated in Section 1, we will show that our conditions on the spectrum of $\mathcal{L}$ imply (2.3). This will lead to the following theorem, which is our main result on stability.

Theorem 2.5 Suppose that $\Re \lambda<0$ for any eigenvalue $\lambda$ of $\mathcal{L}$, and that any function $V \in \mathcal{D}_{0}^{1,2}\left(\bar{\Omega}^{c}\right)^{3} \cap W_{\text {loc }}^{2,1}\left(\bar{\Omega}^{c}\right)^{3}$ with $D^{2} V \in L^{2}\left(\bar{\Omega}^{c}\right)^{27}$ and satisfying the equations

$$
\operatorname{div} V=0, \quad \mathcal{P}_{2}\left(\Delta V-\tau \partial_{1} V+\mathcal{B}(V)\right)=0
$$

vanishes. Then there is $\delta>0$ such that for any $u_{0} \in H_{2}\left(\bar{\Omega}^{c}\right) \cap \mathcal{D}_{0, \sigma}^{1,2}\left(\bar{\Omega}^{c}\right)$ with $\left\|u_{0}\right\|_{1,2} \leq \delta$, there exists a unique function $u \in L_{\text {loc }}^{2}([0, \infty), \mathcal{D}(\mathcal{L})) \cap W_{\text {loc }}^{1,2}\left([0, \infty), H_{2}\left(\bar{\Omega}^{c}\right)\right)$ such that (2.4) holds. This function satisfies (1.10).

In view of Lemma 4.1, we have $\mathcal{B}(V) \in L^{2}\left(\bar{\Omega}^{c}\right)^{3}$ for a function $V$ as in (2.5), so that the expression $\mathcal{P}_{2}\left(\Delta V-\tau \partial_{1} V+\mathcal{B}(V)\right)$ is well defined. However, such a function need not belong to $\mathcal{D}(\mathcal{L})$ because it is not required to be in $L^{2}(\bar{\Omega})^{3}$. Therefore, in view of the definition of $\mathcal{L}$, if there were a nonvanishing solution $V$ to $(2.5)$ with properties as stated in Theorem 2.5, the value $\lambda=0$ might be considered as a generalized eigenvalue of $\mathcal{L}$ 
Let us indicate the structure of the work at hand. The next section (Section 3) consists of a collection of auxiliary results, most of them well known and stated for the convenience of the reader. When we cannot give a direct reference for a known result, we will in some cases sketch a proof, again for the convenience of the reader. There are three theorems (Theorem 3.10, 3.11 and 3.13) which provide results that are new as far as we know. Section 4 also deals with auxiliary results, but is focused on issues related to problem (1.18) and (1.19). Again most results, but not all, are well known. The new results in this section, however, are just technical observations adapting known theory to our purposes. Next (Section 5) we consider regularity of the Oseen resolvent system in the whole space $\mathbb{R}^{3}$. In Section 6, we derive a rather general uniqueness theorem for solutions to (1.18) (Theorem 6.2). Once uniqueness is established, we are able to derive estimates of solutions to (1.18) for small values of $|\lambda|$ (Section 7). These Oseen resolvent estimates (Theorem 7.1 -7.3 ) are the key element of our theory. They are exploited in Section 8 in order to derive upper bounds for solutions of the perturbed Oseen resolvent problem (1.19). Once these bounds are available, we are able to rerun the functional analytic mechanism already used in [12], which reduces Theorem 2.5 to Theorem 2.4 (Section 9).

\section{Various auxiliary results.}

$\langle$ theoremT3.10〉

We start by indicating some properties of Sobolev spaces.

Theorem 3.1 ([24, Lemma II.6.1]) Let $p \in(1, \infty), A \subset \mathbb{R}^{3}$ open, bounded, with Lipschitz boundary. Let $B \in\left\{A, \bar{A}^{c}, \mathbb{R}^{3}\right\}$, and $V \in W_{\text {loc }}^{1,1}(B)$ with $\nabla V \in L^{p}(B)^{3}$. Then $V \in W^{1, p}(A)$ in the case $B=A, V \in W_{\text {loc }}^{1, p}\left(\mathbb{R}^{3}\right)$ in the case $B=\mathbb{R}^{3}$, and $V \mid \bar{A}^{c} \cap B_{R} \in$ $W^{1, p}\left(\bar{A}^{c} \cap B_{R}\right)$ for any $R \in(0, \infty)$ with $\bar{A} \subset B_{R}$ if $B=\bar{A}^{c}$.

${ }^{\langle\text {lemmaL3.10〉 }}$ Lemma 3.1 Let $p, q \in(1, \infty)$. If $V \in L^{q}\left(\mathbb{R}^{3}\right) \cap L^{p}\left(\mathbb{R}^{3}\right)$, there is a sequence $\left(\phi_{n}\right)$ in $C_{0}^{\infty}\left(\mathbb{R}^{3}\right)$ with $\left\|V-\phi_{n}\right\|_{s} \rightarrow 0$ for $s \in\{p, q\}$.

Let $V \in W_{\text {loc }}^{1,1}\left(\mathbb{R}^{3}\right) \cap L^{p}\left(\mathbb{R}^{3}\right)$ with $\nabla V \in L^{q}\left(\mathbb{R}^{3}\right)^{3}$. Then there is a sequence $\left(\psi_{n}\right)$ in $C_{0}^{\infty}\left(\mathbb{R}^{3}\right)$ such that $\left\|V-\psi_{n}\right\|_{p} \rightarrow 0$ and $\left\|\nabla V-\nabla \psi_{n}\right\|_{q} \rightarrow 0$

$\langle$ theoremT3.20〉

Proof: Use Friedrich's mollifier.

Theorem 3.2 ([23, p. 149-150]) Let $p \in(1, \infty)$ and $V \in W_{0}^{1, p}\left(\bar{\Omega}^{c}\right)^{3}$ with div $V=0$. Then there is a sequence $\left(\phi_{n}\right)$ in $C_{0}^{\infty}\left(\bar{\Omega}^{c}\right)^{3}$ with div $\phi_{n}=0$ for $n \in \mathbb{N}$ and $\left\|\phi_{n}-V\right\|_{1, p} \rightarrow 0$.

It is perhaps not so well known that $L^{q}$ is compactly imbedded in $W^{-1, q}$ :

$\langle$ theoremT3.30〉

Theorem 3.3 ([24, Theorem II.5.3]) Let $A \subset \mathbb{R}^{3}$ be open and bounded, $q \in(1, \infty)$, and $\left(V_{n}\right)$ a bounded sequence in $L^{q}(A)$. Then there is $V \in L^{q}(A)$ and a subsequence $\left(W_{n}\right)$ of $\left(V_{n}\right)$ such that $\left\|W_{n}-V\right\|_{-1, q} \rightarrow 0$.

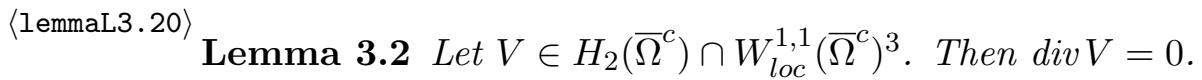

Proof: Take a sequence $\left(\phi_{n}\right)$ in $C_{0}^{\infty}\left(\bar{\Omega}^{c}\right)^{3}$ with $\operatorname{div} \phi_{n}=0$ for $n \in \mathbb{N}$ and $\left\|\phi_{n}-V\right\|_{2} \rightarrow 0$. Let $\varphi \in C_{0}^{\infty}\left(\bar{\Omega}^{c}\right)$. Then $\int_{\bar{\Omega}^{c}} \operatorname{div} V \varphi d x=-\int_{\bar{\Omega}^{c}} V \nabla \varphi d x=\lim _{n \rightarrow \infty}-\int_{\bar{\Omega}^{c}} \phi_{n} \nabla \varphi d x=0$.

$\langle$ theoremT3.40〉 
Theorem 3.4 Let $A \subset \mathbb{R}^{3}$ be either an open bounded nonempty set with Lipschitz boundary, or $A=\emptyset$. Let $q \in(1,3)$ and $V \in W_{\text {loc }}^{1,1}\left(\bar{A}^{c}\right)$ with $\nabla V \in L^{q}\left(\bar{A}^{c}\right)^{3}$. Then there is $V^{*} \in \mathbb{R}$ such that $V-V^{*} \in L^{3 q /(3-q)}\left(\bar{A}^{c}\right)$, and the following five assertion are equivalent:

1.) $V \in L^{r}\left(\bar{A}^{c}\right)$ for some $r \in(1, \infty)$; 2.) $V \in L^{3 q /(3-q)}\left(\bar{A}^{c}\right)$; 3.) $\int_{\partial B_{1}}|V(R x)|^{q} d o_{x} \rightarrow$ $0(R \rightarrow \infty)$; 4.) $\int_{\partial B_{1}}|V(R x)| d o_{x} \rightarrow 0(R \rightarrow \infty)$; 5.) $V^{*}=0$.

If one - and therefore everyone - of these assertions holds, the ensuing inequality is valid:

$$
\|V\|_{3 q /(3-q)} \leq \mathfrak{C}(q, A)\|\nabla V\|_{q}
$$

Proof: According to [24, Lemma II.6.3], the relation $\nabla V \in L^{q}\left(\bar{A}^{c}\right)^{3}$ implies there is $V^{*} \in \mathbb{R}$ with $\int_{\partial B_{1}} \mid\left(V(R x)-\left.V^{*}\right|^{q} d o_{x} \rightarrow 0(R \rightarrow \infty)\right.$. Moreover, by [24, Theorem II.6.1], we have $V-V^{*} \in L^{3 q /(3-q)}\left(\bar{A}^{c}\right)$. Starting from these relations, we showed elsewhere ([10, Lemma 2.4], [11, Lemma 2.1]) that assertion 2.) follows from 1.). Suppose that assertion 3.) holds. Then we get from the convergence relation at the beginning of this proof that $V^{*}=0$, so assertion 2.) holds because $V-V^{*} \in L^{3 q /(3-q)}\left(\bar{A}^{c}\right)$. If assertion 2.) is valid, we may deduce from the preceding relation that $V^{*}=0$, so 3.) follows from the convergence result at the beginning of this proof. Suppose that assertion 4.) holds. Then we may choose a sequence $\left(R_{n}\right)$ in $(0, \infty)$ such that $R_{n} \rightarrow \infty$ and $V\left(R_{n} x\right) \rightarrow 0(n \rightarrow \infty)$ for a. e. $x \in \partial B_{1}$. But again by the convergence result at the beginning of this proof, there is a subsequence $\left(S_{n}\right)$ of $\left(R_{n}\right)$ such that $\left|V\left(S_{n} x\right)-V^{*}\right|^{q} \rightarrow 0$ for a. e. $x \in \partial B_{1}$. Recalling the choice of $\left(R_{n}\right)$, we may conclude that $V^{*}=0$, so assertion 3.) follows by referring once more to the beginning of this proof. Obviously 3.) implies 4.). Inequality (3.1) holds according to [24, Theorem II.6.1].

As a consequence of the preceding theorem, we get that $C_{0}^{\infty}\left(\mathbb{R}^{3}\right)^{3} \subset \widetilde{\mathcal{D}}_{0}^{-1, p}\left(\mathbb{R}^{3}\right)^{3}$ if $p>3 / 2$ : corollaryC3.15>

Corollary 3.1 Let $p \in(3 / 2, \infty)$ and $\phi \in C_{0}^{\infty}\left(\mathbb{R}^{3}\right)^{3}$. Then $\phi \in \widetilde{\mathcal{D}}_{0}^{-1, p}\left(\mathbb{R}^{3}\right)^{3}$.

Proof: Let $R \in(0, \infty)$ with $\operatorname{supp}(\phi) \subset B_{R}$. Since $p^{\prime} \in(1,3)$, we get with (3.1) that $\left|\int_{\mathbb{R}^{3}} \phi \gamma d x\right| \leq\|\phi\|_{(1 / 3+1 / p)^{-1}}\|\gamma\|_{3 p^{\prime} /\left(3-p^{\prime}\right)} \leq\|\phi\|_{(1 / 3+1 / p)^{-1}}\|\nabla \gamma\|_{p^{\prime}}$ for $\gamma \in C_{0}^{\infty}\left(\mathbb{R}^{3}\right)^{3}$.

$\left\langle\right.$ lemmaL3. 30 ${ }^{2}$ Lemma 3.3 Let $q, r \in(1, \infty), R_{0} \in(0, \infty)$. Then

$$
R^{-1}\left\|V \mid B_{2 R \backslash R}\right\|_{q} \leq C\left(q, r, R_{0}\right)\left(\left\|V\left|B_{R}^{c}\left\|_{r}+\right\| \nabla V\right| B_{R}^{c}\right\|_{q}+R^{-1}\|V\|_{r}+R^{-1}\|\nabla V\|_{q}\right)
$$

for $R \in\left(R_{0}, \infty\right), V \in W_{l o c}^{1,1}\left({\overline{B_{R_{0}}}}^{c}\right) \cap L^{r}\left({\overline{B_{R_{0}}}}^{c}\right)$ with $\nabla V \in L^{q}\left({\overline{B_{R_{0}}}}^{c}\right)^{3}$.

Proof: We proceed as in [23, p. 177-178]. Take $R$ and $V$ as in the lemma. First suppose that $q<3$. Then Hölder's inequality yields $\left\|V\left|B_{2 R} \backslash B_{R}\left\|_{q} \leq \mathfrak{C} R\right\| V\right| B_{R}^{c}\right\|_{3 q /(3-q)}$. On the other hand, a scaling argument and Theorem 3.4 with $A=B_{R_{0}}$ imply

$$
\begin{aligned}
& \left\|V\left|B_{R}^{c}\left\|_{3 q /(3-q)}=\left(R / R_{0}\right)^{(3-q) / q}\right\| V\left(\left(R / R_{0}\right) \cdot\right)\right|{\overline{B_{R_{0}}}}^{c}\right\|_{3 q /(3-q)} \\
& \leq C\left(R_{0}, q\right)\left(R / R_{0}\right)^{(3-q) / q}\left\|\nabla\left[V\left(\left(R / R_{0}\right) \cdot\right) \mid{\overline{B_{R_{0}}}}^{c}\right]\right\|_{q}=C\left(R_{0}, q\right)\left\|\nabla V \mid B_{R}^{c}\right\|_{q} .
\end{aligned}
$$

Thus, in the case $q<3$, we have found that $\left\|V\left|B_{2 R} \backslash B_{R}\left\|_{q} \leq C\left(R_{0}, q\right) R\right\| \nabla V\right| B_{R}^{c}\right\|_{q}$. Next suppose that $q \geq 3$ and $q \leq r$. Since we then have $1 / q-1 / r \geq 0$, we get by Hölder's inequality that $\left\|V\left|B_{2 R} \backslash B_{R}\left\|_{q} \leq C R^{3(1 / q-1 / r)}\right\| V\right| B_{R}^{c}\right\|_{r}$. But $q \geq 3$ and $R \geq R_{0}$, so we may conclude that $\left\|V\left|B_{2 R} \backslash B_{R}\left\|_{q} \leq C R_{0}^{3(1 / q-1 / r-1 / 3)} R\right\| V\right| B_{R}^{c}\right\|_{r}$. 
Finally suppose tha $q \geq 3$ and $q>r$. Then $a:=(1 / q-1 / r) /(1 / q-1 / r-1 / 3) \in(0,1)$. Obviously $1 / q=a(1 / q-1 / 3)+(1-a) / r$, so by $\left[23\right.$, Lemma $\left.2.2^{\prime}\right]$, we obtain $\|W\|_{q} \leq$ $C(q, r)\|\nabla W\|_{q}^{a}\|W\|_{r}^{1-a} \leq C(q, r)\left(\|\nabla W\|_{q}+\|W\|_{r}\right)$ for $W \in W_{l o c}^{1,1}\left(\mathbb{R}^{3}\right) \cap L^{r}\left(\mathbb{R}^{3}\right)$ with $\nabla W \in L^{q}\left(\mathbb{R}^{3}\right)^{3}$. Now we split $V$ into a sum of a $W_{l o c}^{1,1}$-function on $\mathbb{R}^{3}$ and a $W_{l o c}^{1,1}$-function on a bounded domain. To this end, we set $B:=B_{R_{0}+1} \backslash \overline{B_{R_{0}}}$, and take $\varphi \in C^{\infty}\left(\mathbb{R}^{3}\right)$ with $\varphi \mid B_{R_{0}+3 / 4}^{c}=1$ and $\varphi \mid B_{R_{0}+1 / 4}=0$. Obviously $\varphi V \in W_{l o c}^{1,1}\left(\mathbb{R}^{3}\right) \cap L^{r}\left(\mathbb{R}^{3}\right)$. According to Theorem 3.1, we have $V \mid B \in W^{1, q}(B)$, so $\nabla(\varphi V) \in L^{q}(B)$. Now the above inequality involving the parameter $a$ yields $\|\varphi V\|_{q} \leq C\left(q, r, R_{0}\right)\left(\|\nabla V\|_{q}+\|V \mid B\|_{q}+\|V\|_{r}\right)$. Hence

$$
\|V\|_{q} \leq\|\varphi V\|_{q}+\|(1-\varphi) V\|_{q} \leq C\left(q, r, R_{0}\right)\left(\|\nabla V\|_{q}+\|V \mid B\|_{q}+\|V\|_{r}\right) .
$$

But the term $\|V \mid B\|_{q}$ my be estimated by Poincaré's inequality for functions with mean value zero. In fact, abbreviate $m:=|B|^{-1} \int_{B} V d x$. Then $\left\|V\left|B\left\|_{q} \leq\right\|(V-m)\right| B\right\|_{q}+$ $|m||B|^{1 / q}$, with $\|(V-m) \mid B\|_{q}$ bounded by $C\left(q, R_{0}\right)\|\nabla V \mid B\|_{q}$, whereas $|m||B|^{1 / q}$ may be estimated by $|B|^{-1+1 / q}\|V \mid B\|_{1}$, and thus by $C\left(q, r, R_{0}\right)\|V\|_{r}$. Therefore from (3.2), $\|V\|_{q} \leq C\left(r, R_{0}\right)\left(\|\nabla V\|_{q}+\|V\|_{r}\right)$. The lemma follows from the preceding inequality and from our estimates of $\left\|V \mid B_{2 R} \backslash B_{R}\right\|_{q}$ in the cases $q<3$ and $q \geq 3, q \leq r$, respectively.

$\langle$ theoremT3.60〉

Next we collect some properties of $\mathcal{D}_{0}^{1,2}\left(\bar{\Omega}^{c}\right)$ and $\mathcal{D}_{0}^{-1,2}\left(\bar{\Omega}^{c}\right)$.

Theorem 3.5 The mapping $(V, W) \mapsto \int_{\bar{\Omega}^{c}} \nabla V \cdot \nabla W d x$ is a scalar product on $\mathcal{D}_{0}^{1,2}\left(\bar{\Omega}^{c}\right)$, and $\mathcal{D}_{0}^{1,2}\left(\bar{\Omega}^{c}\right)$ equipped with this scalar product is a Hilbert space, with associated norm $V \mapsto\|\nabla V\|_{2}$. Moreover the inequality $\|V\|_{6} \leq \mathfrak{C}\|\nabla V\|_{2}$ holds for $V \in \mathcal{D}_{0}^{1,2}\left(\bar{\Omega}^{c}\right)$, and the set $C_{0}^{\infty}\left(\bar{\Omega}^{c}\right)$ is dense in $\mathcal{D}_{0}^{1,2}\left(\bar{\Omega}^{c}\right)$.

Proof: For the Hilbert space property, we refer to [24, p. 105] or [49, Theorem I.2.2, I.2.8]. The inequality $\|V\|_{6} \leq \mathfrak{C}\|\nabla V\|_{2}$ holds according to (3.1). As concerns density of $C_{0}^{\infty}\left(\bar{\Omega}^{c}\right)$ in $\mathcal{D}_{0}^{1,2}\left(\bar{\Omega}^{c}\right)$, we refer to Theorem 3.4 and [23, Theorem II.7.1], or to [49, Theorem I.2.8].

$\langle$ theoremT3.70〉

Theorem 3.6 Let $g \in L_{\text {loc }}^{1}\left(\bar{\Omega}^{c}\right)^{3}$ and put $G_{g}(\varphi):=\int_{\bar{\Omega}^{c}} g \cdot \varphi d x$ for $\varphi \in C_{0}^{\infty}\left(\bar{\Omega}^{c}\right)^{3}$. Suppose that $\|g\|_{-1,2}^{*}:=\sup \left\{G_{g}(\varphi) /\|\nabla \varphi\|_{2}: \varphi \in C_{0}^{\infty}\left(\bar{\Omega}^{c}\right)^{3}, \varphi \neq 0\right\}<\infty$. Then there is a unique element $F_{g} \in \mathcal{D}_{0}^{-1,2}\left(\bar{\Omega}^{c}\right)^{3}$ with $F_{g} \mid C_{0}^{\infty}\left(\bar{\Omega}^{c}\right)^{3}=G_{g}$, and the relation $\|g\|_{-1,2}^{*}=\left\|F_{g}\right\|_{-1,2}$ holds. In this case, we write $g$ instead of $F_{g}$, and $g \in \mathcal{D}_{0}^{-1,2}\left(\bar{\Omega}^{c}\right)^{3}$ instead of $\|g\|_{-1,2}^{*}<\infty$.

If $g \in L^{6 / 5}\left(\bar{\Omega}^{c}\right)^{3}$, then $g \in \mathcal{D}_{0}^{-1,2}\left(\bar{\Omega}^{c}\right)^{3}, F_{g}(\varphi)=\int_{\bar{\Omega}^{c}} g \cdot \varphi d x$ for any $\varphi \in \mathcal{D}_{0}^{1,2}\left(\bar{\Omega}^{c}\right)^{3}$, and $\|g\|_{-1,2} \leq \mathfrak{C}(\Omega)\|g\|_{6 / 5}$.

For any $h \in \mathcal{D}_{0}^{-1,2}\left(\bar{\Omega}^{c}\right)^{3}$, there is a sequence $\left(\varphi_{n}\right)$ in $C_{0}^{\infty}\left(\bar{\Omega}^{c}\right)^{3}$ with $\left\|h-\varphi_{n}\right\|_{-1,2} \rightarrow 0$.

Proof: Hahn-Banach's theorem, the density of $C_{0}^{\infty}\left(\bar{\Omega}^{c}\right)$ in $\mathcal{D}_{0}^{1,2}\left(\bar{\Omega}^{c}\right)$ (Theorem 3.5) and the definition of $\|g\|_{-1,2}^{*}$ yield existence of $F_{g} \in \mathcal{D}_{0}^{-1,2}\left(\bar{\Omega}^{c}\right)^{3}$ with $F_{g} \mid C_{0}^{\infty}\left(\bar{\Omega}^{c}\right)^{3}=G_{g}$. Uniqueness of $F_{g}$ follows again from the density of $C_{0}^{\infty}\left(\bar{\Omega}^{c}\right)$ in $\mathcal{D}_{0}^{1,2}\left(\bar{\Omega}^{c}\right)$. By Hölder's inequality and the estimate $\|\varphi\|_{6} \leq \mathfrak{C}\|\nabla \varphi\|_{2}$ (Theorem 3.5), we get that $\int_{\bar{\Omega}^{c}}|g \cdot \varphi| d x \leq$ $\|g\|_{6 / 5}\|\nabla \varphi\|_{2}$ for $\varphi \in C_{0}^{\infty}\left(\bar{\Omega}^{c}\right)^{3}$, so the claim of the theorem related to the case $g \in$ $L^{6 / 5}\left(\bar{\Omega}^{c}\right)^{3}$ is true. The last statement of the theorem follows by [23, Theorem II.6.5].

Lemma 3.4 Let $G \in C_{0}^{\infty}\left(\bar{\Omega}^{c}\right)^{3}$. Then $\|G\|_{-1,2} \leq \mathfrak{C}\left\|G \mid \bar{\Omega}^{c}\right\|_{*}$. 
Proof: Let $\varphi \in C^{\infty}\left(\mathbb{R}^{3}\right)$ with $\varphi\left|B_{S}=0, \varphi\right| B_{S+1}^{c}=1$ and $0 \leq \varphi \leq 1$. Let $\gamma \in$ $C_{0}^{\infty}\left(\mathbb{R}^{3}\right)^{3}$. Then $\varphi \gamma \in C_{0}^{\infty}\left(\bar{\Omega}^{c}\right)^{3}$, so by splitting $G$ into the sum $(1-\varphi) G+\varphi G$, , we get $\left|\int_{\mathbb{R}^{3}} G \cdot \gamma d x\right| \leq\left\|G\left|\Omega_{S+1}\left\|_{2}\right\|(1-\varphi) \gamma\left\|_{2}+\right\| G\right| \bar{\Omega}^{c}\right\|_{-1,2}\|\nabla(\varphi \gamma)\|_{2}$. Put $B:=B_{S+3 / 4} \backslash \overline{B_{S}}$. Then by Poincaré's inequality on $B_{S+1}$, we see that $\|(1-\varphi) \gamma\|_{2} \leq \mathfrak{C}\left(\|\gamma \mid B\|_{2}+\|\nabla \gamma\|_{2}\right)$. Moreover $\|\nabla(\varphi \gamma)\|_{2} \leq \mathfrak{C}\left(\|\gamma \mid B\|_{2}+\|\nabla \gamma\|_{2}\right)$. But the estimate $\left\|\gamma\left|B\left\|_{2} \leq C(S)\right\| \gamma\right| B\right\|_{6}$ and Theorem 3.4 with $A={\overline{B_{S}}}^{c}$ imply $\|\gamma \mid B\|_{2} \leq \mathfrak{C}\|\nabla \gamma\|_{2}$. Collecting the previous estimates yields the lemma.

We turn to the boundary value problem $\operatorname{div} V=G$ in $B, V \mid \partial B=0$, for annular domains $B \subset \mathbb{R}^{3}$.

Theorem 3.7 ([6, Theorem 2.4]) Let $R, \widetilde{R} \in(0, \infty)$ with $R<\widetilde{R}$, and put $B:=$ $B_{\widetilde{R}} \backslash \overline{B_{R}}$. Then, for any $q \in(1, \infty)$ and $m \in\{0,1\}$, there is a linear operator $\mathfrak{D}:=$ $\mathfrak{D}(q, m, R, \widetilde{R})$ from $\left\{g \in W_{0}^{m, q}(B): \int_{B} g d x=0\right\}$ into $W_{0}^{m+1, q}(B)^{3}$ such that div $\mathfrak{D}(g)=$ $g$ and $\|\mathfrak{D}(g)\|_{m+1, q} \leq C(q, m, R, \widetilde{R})\|g\|_{m, q}$ for $g \in W_{0}^{m, q}(B)$ with $\int_{B} g d x=0$.

Moreover, for $p, m$ as before, and for $g \in C_{0}^{\infty}(B)$ with $\int_{B} g d x=0$, the function $\mathfrak{D}(g)$ (or more precisely: the zero extension of $\mathfrak{D}(g \mid B)$ to $\left.\mathbb{R}^{3}\right)$ belongs to $C_{0}^{\infty}(B)^{3}$.

Finally, if $p, q \in(1, \infty), m, n \in\{0,1\}$ and $g \in C_{0}^{\infty}(B)$ with $\int_{B} g d x=0$, then the functions $\mathfrak{D}(p, m, R, \widetilde{R})(g)$ and $\mathfrak{D}(q, n, R, \widetilde{R})(g)$ coincide.

The ensuing lemma deals with solutions to the boundary value problem $\operatorname{div} V=G$ in $B, V \mid \partial B=0$, with $B$ belonging to the set $\left\{B_{2 n} \backslash B_{n}:, n \in \mathbb{N}\right\}$ of annular domains. The lemma indicates how the $L^{p}$-norm of these solutions depends on $n$.

$\langle$ lemmaL3.50〉

Lemma 3.5 Let $q \in(1, \infty), \nu \in\{0,1\}, C_{0}>0, \mathcal{W}_{n}:=\left\{g \in W_{0}^{\nu, q}\left(B_{2 n} \backslash \overline{B_{n}}\right)\right.$ : $\left.\int_{B_{2 n} \backslash \overline{B_{n}}} g d x=0\right\}$ for $n \in \mathbb{N}, \mathfrak{D}: \mathcal{W}_{1} \mapsto W_{0}^{\nu+1, q}\left(B_{2} \backslash \overline{B_{1}}\right)^{3}$ a mapping such that div $\mathfrak{D}(g)=$ $g$ and $\|\mathfrak{D}(g)\|_{1+\nu, q} \leq C_{0}\|g\|_{\nu, q}$ for $g \in \mathcal{W}_{1}$. For $n \in \mathbb{N}, g \in \mathcal{W}_{n}, x \in B_{2 n} \backslash \overline{B_{n}}$, set $\mathfrak{D}_{n}(g)(x):=n \mathfrak{D}(g(n \cdot))((1 / n) x)$.

Then, for $n \in \mathbb{N}, g \in \mathcal{W}_{n}$, we have $\mathcal{D}_{n}(g) \in W_{0}^{\nu+1, q}\left(B_{2 n} \backslash \overline{B_{n}}\right)^{3}$, $\operatorname{div} \mathfrak{D}_{n}(g)=g$ and $\left\|\partial^{\alpha} \mathfrak{D}_{n}(g)\right\|_{q} \leq C_{0} n^{1-|\alpha|}\|g\|_{q}$ for $\alpha \in \mathbb{N}_{0}^{3}$ with $|\alpha| \leq 1$ in the case $\nu=0$, as well as $\left\|\partial_{l} \partial_{m} \mathfrak{D}_{n}(g)\right\|_{q} \leq C_{0}\|g\|_{1, q}$ for $l, m \in\{1,2,3\}$ in the case $\nu=1$.

Proof: Direct calculation, via scaling.

Next we recall some results related to Poisson's equation $\Delta V=G$.

$\langle$ theoremT3.90〉

Theorem 3.8 (Weyl's lemma) Let $A \subset \mathbb{R}^{3}$ be open and $V \in L_{\text {loc }}^{1}(A)$ such that the integral $\int_{A} V \Delta \varphi d x$ vanishes for any $\varphi \in C_{0}^{\infty}(A)$. Then $V \in C^{\infty}(A)$ and $\Delta V=0$.

Proof: An elementary proof may be found in [49, Appendix].

The consequence of Theorem 3.8 we have in mind is the ensuing corollary, which in principle is well known, but which we state and prove because we do not know a direct reference.

Corollary 3.2 Let $A \subset \mathbb{R}^{3}$ be open and connected, $\Pi \in W_{\text {loc }}^{1,1}(A)$ with $\nabla \Pi=0$. Then there is $c \in \mathbb{R}$ with $\Pi(x)=c$ for a. e. $x \in A$.

Proof: Theorem 3.8 yields $\Pi \in C_{0}^{\infty}(A)$.

The ensuing theorem deals with the Newton potential. Since some subtleties of this 
potential play an important role in what follows, we state them here. Concerning proofs, they are, of course, well known. We only mention for completeness that the relations in (3.3) follow by some integration by parts and Lebesgue's theorem, the estimates in (3.5) and (3.6) are a consequence of Hardy-Littlewood-Sobolev's inequality, whereas inequality (3.4) may deduced from Calderon-Zygmund's inequality. The other claims of the theorem follow from these inequalities via density arguments. Lemma 3.1 is useful in this context.

Theorem 3.9 Let $\phi \in C_{0}^{\infty}\left(\mathbb{R}^{3}\right)$. Then the integral $\int_{\mathbb{R}^{3}}\left|\partial^{\alpha} N(x-y) \partial^{\beta} \phi(y)\right|$ dy is finite for $x \in \mathbb{R}^{3}, \alpha \in \mathbb{N}_{0}^{3}$ with $|\alpha| \leq 1, \beta \in \mathbb{N}_{0}^{3}$, and

$$
\begin{aligned}
& N * \phi \in C^{\infty}\left(\mathbb{R}^{3}\right), \quad \partial^{\beta}(N * \phi)=N * \partial^{\beta}(\phi) \text { for } \beta \in \mathbb{N}_{0}^{3}, \\
& \partial_{l}(N * \phi)=\left(\partial_{l} N\right) * \phi \text { for } 1 \leq l \leq 3, \quad \Delta(N * \phi)=0, \\
& \left\|\partial_{l} \partial_{m}(N * \phi)\right\|_{q} \leq C(q)\|\phi\|_{q} \text { for } 1 \leq l, m \leq 3, \quad q \in(1, \infty) .
\end{aligned}
$$

Let $q \in(1,3 / 2), \phi \in L^{q}\left(\mathbb{R}^{3}\right)$. Then

$$
\|N *|\phi|\|_{3 q /(3-2 q)} \leq C(q)\|\phi\|_{q},
$$

in particular $N * \phi \in L^{3 q /(3-2 q)}\left(\mathbb{R}^{3}\right)$ and $\int_{\mathbb{R}^{3}}|N(x-y) \phi(y)| d y<\infty$ for a. e. $x \in \mathbb{R}^{3}$. Moreover $N * \phi \in W_{l o c}^{2,1}\left(\mathbb{R}^{3}\right)$

Let $q \in(1,3), \phi \in L^{q}\left(\mathbb{R}^{3}\right)$. Then

$$
\left\|\left|\partial_{l} N\right| *|\phi|\right\|_{3 q /(3-q)} \leq C(q)\|\Phi\|_{q} \text { for } 1 \leq l \leq 3,
$$

in particular $\partial_{l} N * \phi \in L^{3 q /(3-q)}\left(\mathbb{R}^{3}\right)$ and $\int_{\mathbb{R}^{3}}\left|\partial_{l} N(x-y)\right||\phi(y)| d y<\infty$ for a. e. $x \in \mathbb{R}^{3}$. Moreover $\left(\partial_{l} N\right) * \phi \in W_{l o c}^{1,1}\left(\mathbb{R}^{3}\right)$ and $\operatorname{div}\left(\left(\partial_{l} N\right) * \phi\right)_{1 \leq l \leq 3}=\phi$.

If $q \in(1,3 / 2), \phi \in L^{q}\left(\mathbb{R}^{3}\right)$, then $\partial_{l}(N * \phi)=\left(\partial_{l} N\right) * \phi(1 \leq l \leq 3)$. If $q \in(1,3)$, $p \in$ $(1, \infty), \phi \in L^{q}\left(\mathbb{R}^{3}\right) \cap L^{p}\left(\mathbb{R}^{3}\right)$, then

$$
\left\|\partial_{m}\left(\left(\partial_{l} N\right) * \phi\right)\right\|_{p} \leq C(p)\|\phi\|_{p}(1 \leq l, m \leq 3) .
$$

In addition $\partial_{l}(N * \phi) \in L^{r}\left(\mathbb{R}^{3}\right)$ for any $r \in(3 / 2, \infty)$ if $\phi \in L^{q}\left(\mathbb{R}^{3}\right)$ for any $q \in(1,3)$, and $\partial_{m} \partial_{l}(N * \phi) \in L^{r}\left(\mathbb{R}^{3}\right)$ for any $r \in(1, \infty)$ if $\phi \in L^{q}\left(\mathbb{R}^{3}\right)$ for any $q \in(1, \infty)$.

Corollary 3.3 Let $p \in(1, \infty), \phi \in \cup_{q \in(1,3)} L^{q}\left(\mathbb{R}^{3}\right) \cap \widetilde{\mathcal{D}}_{0}^{-1, p}\left(\mathbb{R}^{3}\right), \quad l \in\{1,2,3\}$. Then $\left\|\left(\partial_{l} N\right) * \phi\right\|_{p} \leq C(p)\|\phi\|_{-1, p}$.

Proof: We simplify the argument from [23, p. 394-396]. By the assumptions on $\phi$, we may choose $q \in(1,3)$ with $\phi \in L^{q}\left(\mathbb{R}^{3}\right)$, so $\left(\partial_{l} N\right) * \phi \in L^{3 q /(3-q)}\left(\mathbb{R}^{3}\right)$ (Theorem 3.9). Let $\psi \in C_{0}^{\infty}\left(\mathbb{R}^{3}\right)$. In view of (3.6), we may apply Fubini's theorem, to obtain $\int_{\mathbb{R}^{3}}\left(\left(\partial_{l} N\right) *\right.$ $\phi) \psi d x=\int_{\mathbb{R}^{3}}\left(\left(\partial_{l} N\right) * \psi\right) \phi d x$. Since $q<3$, we have $q^{\prime}>3 / 2$, so $\left(\partial_{l} N\right) * \psi \in L^{q^{\prime}}\left(\mathbb{R}^{3}\right)$ and also $\nabla\left(\left(\partial_{l} N\right) * \psi\right) \in L^{p^{\prime}}\left(\mathbb{R}^{3}\right)$ by (3.3) and the last two assertions of Theorem 3.9. Thus, by Lemma 3.1 , we may choose a sequence $\left(\gamma_{n}\right)$ in $C_{0}^{\infty}\left(\mathbb{R}^{3}\right)$ such that $\left\|\left(\partial_{l} N\right) * \psi-\gamma_{n}\right\|_{q^{\prime}} \rightarrow 0$ and $\left\|\nabla\left(\left(\partial_{l} N\right) * \psi\right)-\nabla \gamma_{n}\right\|_{p^{\prime}} \rightarrow 0$. We now find

$$
\begin{aligned}
& \left|\int_{\mathbb{R}^{3}}\left(\left(\partial_{l} N\right) * \phi\right) \psi d x\right| \leq \limsup _{n \rightarrow \infty}\left|\int_{\mathbb{R}^{3}} \gamma_{n} \phi d x\right| \leq \limsup _{n \rightarrow \infty}\|\phi\|_{-1, p}\left\|\nabla \gamma_{n}\right\|_{p^{\prime}} \\
& \leq\|\phi\|_{-1, p}\left\|\nabla\left(\left(\partial_{l} N\right) * \psi\right)\right\|_{p^{\prime}} \leq\|\phi\|_{-1, p}\|\| \psi \|_{p^{\prime}},
\end{aligned}
$$


where the last inequality follows from (3.7).

We now show that a function from $H_{2}\left(\mathbb{R}^{3}\right) \cap \widetilde{\mathcal{D}}_{0}^{-1,2}\left(\mathbb{R}^{3}\right)^{3}$ may be approximated simultaneously in $L^{2}\left(\mathbb{R}^{3}\right)^{3}$ and $\widetilde{\mathcal{D}}_{0}^{-1,2}\left(\mathbb{R}^{3}\right)^{3}$ by smooth solenoidal functions. This is a generalization of [23, Lemma VII.4.3], although only for the case $q=2$, but it seems to be new.

Theorem 3.10 Let $\phi \in H_{2}\left(\mathbb{R}^{3}\right) \cap \widetilde{\mathcal{D}}_{0}^{-1,2}\left(\mathbb{R}^{3}\right)^{3}$. Then there is a sequence $\left(\phi_{n}\right)$ in $C_{0}^{\infty}\left(\mathbb{R}^{3}\right)$ with div $\phi_{n}=0(n \in \mathbb{N})$ and $\left\|\phi-\phi_{n}\right\|_{2} \rightarrow 0$ and $\left\|\phi-\phi_{n}\right\|_{-1,2} \rightarrow 0$.

Proof: Since $\phi \in L^{2}\left(\mathbb{R}^{3}\right)^{3}$, we may define $v_{j l}:=\left(\partial_{l} N\right) * \phi_{j}$ for $1 \leq j \leq 3$; see Theorem 3.9. By the same reference, in particular (3.5), we get $v_{j l} \in W_{l o c}^{1,1}\left(\mathbb{R}^{3}\right) \partial_{m} v_{j l} \in L^{2}\left(\mathbb{R}^{3}\right)(1 \leq$ $j, l, m \leq 3), \quad \sum_{l=1}^{3} \partial_{l} v_{j l}=\phi_{j}(1 \leq j \leq 3)$. Corollary 3.3 yields $v_{j l} \in L^{2}\left(\mathbb{R}^{3}\right)$, so we have $v_{j l} \in W^{1,2}\left(\mathbb{R}^{3}\right)(1 \leq j, l \leq 3)$. Since $\phi \in H_{2}\left(\mathbb{R}^{3}\right)$, we may choose a sequence $\left(\psi_{n}\right)$ in $C_{0}^{\infty}\left(\mathbb{R}^{3}\right)^{3}$ with $\operatorname{div} \psi_{n}=0$ for $n \in \mathbb{N}$ and $\left\|\phi-\psi_{n}\right\|_{2} \rightarrow 0$. It follows with (3.7) that

$$
\left\|\partial_{m} v_{j l}-\partial_{m}\left(\left(\partial_{l} N\right) * \psi_{n, j}\right)\right\|_{2} \rightarrow 0 \quad(1 \leq j, l, m \leq 3) .
$$

Next we observe that $\partial_{j}\left(\left(\partial_{l} N\right) * \psi_{n, j}\right)=\partial_{j} \partial_{l}\left(N * \psi_{n, j}\right)=\partial_{l}\left(N * \partial_{j} \psi_{n, j}\right)$ for $1 \leq j, l \leq$ $3, n \in \mathbb{N}$, as follows again by Theorem 3.9, in particular (3.3). Thus $\operatorname{div}\left(\left(\partial_{l} N\right) * \psi_{n, j}\right)=0$ for $l, n$ as before, hence from (3.8)

$$
\operatorname{div}\left(v_{j l}\right)_{1 \leq j \leq 3}=0 \text { for } 1 \leq l \leq 3 .
$$

Let $\varphi \in C_{0}^{\infty}\left(B_{1}\right)$ with $\varphi \geq 0, \int_{B_{1}} \varphi d x=1$, and define $\varphi_{\epsilon}(x):=\epsilon^{-3} \varphi(\epsilon x)$ for $x \in$ $\mathbb{R}^{3}, \quad w_{\epsilon}:=\varphi_{\epsilon} * w$ for $\epsilon>0, w \in L_{l o c}^{1}\left(\mathbb{R}^{3}\right)$ (Friedrich's mollifier). By standard properties of this mollifier, by (3.9) and because $v_{j l} \in W^{1,2}\left(\mathbb{R}^{3}\right)$, we get $\left(v_{j, l}\right)_{\epsilon} \in C^{\infty}\left(\mathbb{R}^{3}\right) \cap W^{1,2}\left(\mathbb{R}^{3}\right)$,

$$
\operatorname{div}\left(\left(v_{r l}\right)_{\epsilon}\right)_{1 \leq r \leq 3}=0 \text { for } \epsilon>0, \quad\left\|\partial^{\alpha} v_{j l}-\partial^{\alpha}\left(v_{j l}\right)_{\epsilon}\right\|_{2} \rightarrow 0 \quad(\epsilon \downarrow 0)
$$

$\left(1 \leq j, l \leq 3, \alpha \in \mathbb{N}_{0}^{3}\right.$ with $\left.|\alpha| \leq 1\right)$. Choose some $\gamma \in C_{0}^{\infty}\left(B_{7 / 4}\right)$ with $\gamma \mid B_{5 / 4}=$ $1,0 \leq \gamma \leq 1$. Define $\gamma_{n}(x)=\gamma((1 / n) x)$ for $x \in \mathbb{R}^{3} n \in \mathbb{N}$. Note that $\gamma \mid B_{5 n / 4}=1$ for $n \in \mathbb{N}$. We recall that $\sum_{l=1}^{3} \partial_{l} v_{j l}=\phi_{j}$. Thus the idea now is to approximate $\phi$ by a solenoidal version of $\left(\sum_{l=1}^{3} \partial_{l}\left[\gamma_{n}\left(v_{j l}\right)_{\epsilon}\right]\right)_{1<j<3}$, with $n \in \mathbb{N}$ and $\epsilon>0$ as independent parameters. In order to introduce such a solenoidal version, take $n \in \mathbb{N}, \epsilon>0$ and $l \in\{1,2,3\}$. Then $\nabla \gamma_{n}\left(\left(v_{j l}\right)_{\epsilon}\right)_{1 \leq j \leq 3} \in C_{0}^{\infty}\left(B_{2 n} \backslash \overline{B_{n}}\right)$. This observation and (3.10) yield $\int_{B_{2 n} \backslash \overline{B_{n}}} \nabla \gamma_{n}\left(\left(v_{j l}\right)_{\epsilon}\right)_{1 \leq j \leq 3} d x=0$. Thus, in view of Theorem 3.7 , we may define $\mathcal{D}_{l, n, \epsilon}$ as abbreviation of the function $n \mathcal{D}(2,0,1,2)\left(-\left[\nabla \gamma_{n}\left(\left(v_{j l}\right)_{\epsilon}\right)_{1 \leq j \leq 3}\right](n \cdot)\right)((1 / n) \cdot)$, and then put $\psi_{l, n, \epsilon}:=\gamma_{n}\left(\left(v_{j l}\right)_{\epsilon}\right)_{1 \leq j \leq 3}+\mathcal{D}_{l, n, \epsilon}(1 \leq l \leq 3, n \in \mathbb{N}, \epsilon>0)$. By Theorem 3.7 and Lemma 3.5, we have $\psi_{l, n, \epsilon} \in C_{0}^{\infty}\left(\mathbb{R}^{3}\right)^{3}$ and $\operatorname{div} \psi_{l, n, \epsilon}=0$. Finally put $\phi_{n, \epsilon}:=\sum_{l=1}^{3} \partial_{l} \psi_{l, n, \epsilon}$ for $\epsilon>0, n \in \mathbb{N}$. Then $\phi_{n, \epsilon} \in C_{0}^{\infty}\left(\mathbb{R}^{3}\right)^{3}$ and $\operatorname{div} \phi_{n, \epsilon}=0$ for $\epsilon, n$ as before, as follows from corresponding properties of $\psi_{l, n, \epsilon}$. Using the equation $\sum_{l=1}^{3} \partial_{l} v_{j l}=\phi_{j}(1 \leq j \leq 3)$, we split the difference $\phi_{n, \epsilon, j}-\phi_{j}$ into a sum of twelve parts: $\phi_{n, \epsilon, j}-\phi_{j}=\sum_{i=1}^{4} \sum_{l=1}^{3} \mathfrak{A}_{n, \epsilon, j, l}^{(i)}$ for $n \in \mathbb{N}, \epsilon>0,1 \leq j \leq 3$, with $\mathfrak{A}_{n, \epsilon, j, l}^{(1)}:=\partial_{l} \gamma_{n}\left(v_{j l}\right)_{\epsilon}, \mathfrak{A}_{n, \epsilon, j, l}^{(2)}:=\partial_{l} \mathcal{D}_{l, n, \epsilon, j}, \mathfrak{A}_{n, \epsilon, j, l}^{(3)}:=$ $\gamma_{n}\left(\partial_{l}\left(v_{j l}\right)_{\epsilon}-\partial_{l} v_{j l}\right), \mathfrak{A}_{n, \epsilon, j, l}^{(4)}:=\left(\gamma_{n}-1\right) \partial_{l} v_{j l}$ for $1 \leq l \leq 3$. Similarly, since $\psi_{l, n, \epsilon} \in$ $C_{0}^{\infty}\left(\mathbb{R}^{3}\right)^{3}$, we get for $\zeta \in C_{0}^{\infty}\left(\mathbb{R}^{3}\right)^{3}$, after an integration by parts,

$$
\int_{\mathbb{R}^{3}}\left(\phi_{n, \epsilon, j, l}-\phi_{j}\right) \zeta_{j} d x=\int_{\mathbb{R}^{3}} \sum_{i=1}^{3} \sum_{l=1}^{3} \mathfrak{B}_{n, \epsilon, j, l}^{(i)} \partial_{l} \zeta_{j} d x \quad \text { for } \epsilon>0, n \in \mathbb{N}, 1 \leq j \leq 3,
$$


with $\mathfrak{B}_{n, \epsilon, j, l}^{(1)}:=\gamma_{n}\left(\left(v_{j l}\right)_{\epsilon}-v_{j l}\right), \mathfrak{B}_{n, \epsilon, j, l}^{(2)}:=\mathcal{D}_{l, n, \epsilon, j}, \mathfrak{B}_{n, \epsilon, j, l}^{(3)}:=\left(\gamma_{n}-1\right) v_{j l}$ for $1 \leq l \leq 3$. Let $\kappa \in(0, \infty)$. Due to second relation in (3.10), we may choose $\epsilon_{0}>0$ so small that $\left\|\mathfrak{A}_{n, \epsilon_{0}, j, l}^{(3)}\right\|_{2}+\left\|\mathfrak{B}_{n, \epsilon_{0}, j, l}^{(1)}\right\|_{2} \leq \kappa$ for $1 \leq j, l \leq 3, n \in \mathbb{N}$. Moreover, since $v_{j l} \in W^{1,2}\left(\mathbb{R}^{3}\right)$ and $\left(1-\gamma_{n}\right) \mid B_{n}=0$ for $j, l, n$ as before, we may choose $n_{1} \in \mathbb{N}$ such that $\left\|\mathfrak{A}_{n, \epsilon_{0}, j, l}^{(4)}\right\|_{2}+$ $\left\|\mathfrak{B}_{n, \epsilon_{0}, j, l}^{(3)}\right\|_{2} \leq \kappa$ for $1 \leq j, l \leq 3, n \in \mathbb{N}, n \geq n_{1}$. In addition, with Theorem 3.7, Lemma 3.5 and because $|\nabla \lambda|_{\infty} \leq C / n$ for $n \in \mathbb{N}$, we get that $\left\|\mathfrak{A}_{n, \epsilon_{0}, j, l}^{(2)}\right\|_{2}+\left\|\mathfrak{B}_{n, \epsilon_{0}, j, l}^{(2)}\right\|_{2}$ is bounded by $\mathfrak{C} n\left\|\nabla \gamma\left(\left(v_{k l}\right)_{\epsilon_{0}}\right)_{1 \leq k \leq 3}\right\|_{2}$, and hence by $\mathfrak{C} \max \left\{\left\|\left(v_{k l}\right)_{\epsilon_{0}} \mid B_{n_{0}}^{c}\right\|_{2}: 1 \leq k \leq\right.$ $3\}$ for $n \in \mathbb{N}, 1 \leq j, l \leq 3$. Thus, since $\left(v_{j, l}\right)_{\epsilon_{0}} \in L^{2}\left(\mathbb{R}^{3}\right)$, there is $n_{2} \in \mathbb{N}$ such that $\left\|\mathfrak{A}_{n, \epsilon_{0}, j, l}^{(2)}\right\|_{2}+\left\|\mathfrak{B}_{n, \epsilon_{0}, j, l}^{(2)}\right\|_{2} \leq \kappa$ for $n \in \mathbb{N}, n \geq n_{2}, \quad 1 \leq j, l \leq 3$. Finally, using again that $|\nabla \lambda|_{\infty} \leq C / n$ and $\left(v_{j, l}\right)_{\epsilon_{0}} \in L^{2}\left(\mathbb{R}^{3}\right)$, we may choose $n_{3} \in \mathbb{N}$ with $\left\|\mathfrak{A}_{n, \epsilon_{0}, j, l}^{(1)}\right\|_{2} \leq \kappa$ for $n \in \mathbb{N}, n \geq n_{3}$ and $1 \leq j, l \leq 3$. The preceding estimates taken together yield $\left\|\phi-\phi_{n, \epsilon_{0}, j}\right\|_{2} \leq 12 \kappa$ and $\left|\int_{\mathbb{R}^{3}}\left(\phi-\phi_{n, \epsilon_{0}, j}\right) \zeta_{j} d x\right| \leq 9 \kappa\|\nabla \zeta\|_{2}$ for $1 \leq j \leq 3, \zeta \in C_{0}^{\infty}\left(\mathbb{R}^{3}\right)^{3}$ and $n \in \mathbb{N}$ sufficiently large. Theorem 3.10 follows.

A simplified version of the preceding proof yields a somewhat more direct access to [23, Lemma VII.4.3], which we will also need in the case $q=2$, and therefore state as

Corollary 3.4 Let $\phi \in L^{2}\left(\mathbb{R}^{3}\right) \cap \widetilde{\mathcal{D}}_{0}^{-1,2}\left(\mathbb{R}^{3}\right)$. Then there is a sequence $\left(\phi_{n}\right)$ in $C_{0}^{\infty}\left(\mathbb{R}^{3}\right)$ with $\left\|\phi-\phi_{n}\right\|_{2} \rightarrow 0$ and $\left\|\phi-\phi_{n}\right\|_{-1,2} \rightarrow 0$.

The purpose of Theorem 3.10 and Corollary 3.4 is to serve as tools in order to establish analogous results for the exterior domain case. We elaborate the proof of the analogue of Theorem 3.10 .

Theorem 3.11 Let $G \in H_{2}\left(\bar{\Omega}^{c}\right) \cap \mathcal{D}_{0}^{-1,2}\left(\bar{\Omega}^{c}\right)^{3}$. Then there is a sequence $\left(\Phi_{n}\right)$ in $C_{0}^{\infty}\left(\mathbb{R}^{3}\right)^{3}$ such that div $\Phi_{n}=0$ for $n \in \mathbb{N},\left\|G-\Phi_{n}\right\|_{2} \rightarrow 0$ and $\left\|G-\Phi_{n}\right\|_{-1,2} \rightarrow 0$.

Proof: Fix some function $\varphi \in C_{0}^{\infty}\left(B_{S+7 / 4}\right)$ with $\varphi \mid B_{S+5 / 4}=1$. Choose a sequence $\left(\phi_{n}\right)$ in $C_{0}^{\infty}\left(\bar{\Omega}^{c}\right)^{3}$ with $\operatorname{div} \phi_{n}=0$ for $n \in \mathbb{N}$ and $\left\|G-\phi_{n}\right\|_{2} \rightarrow 0$. Abbreviate $B:=$ $B_{S+2} \backslash \overline{B_{S+1}}$. Then $\int_{B} \nabla \varphi \phi_{n} d x=0$ for $n \in \mathbb{N}$, hence $\int_{B} \nabla \varphi G d x=0$. Therefore, following Theorem 3.7, we may consider the functions $\mathfrak{D}:=\mathfrak{D}(2,0, S+1, S+2)(-\nabla \varphi \cdot G \mid B)$ and $\mathfrak{D}_{n}:=\mathfrak{D}(2,0, S+1, S+2)\left(-\nabla \varphi \cdot \phi_{n} \mid B\right)$ for $n \in \mathbb{N}$. This means in particular that $\mathfrak{D} \in W_{0}^{1,2}(B)^{3}$ and $\mathfrak{D}_{n} \in C_{0}^{\infty}(B)^{3}$. We further get with Theorem 3.7 that $\varphi \phi_{n}+\mathfrak{D}_{n} \in$ $C_{0}^{\infty}\left(\bar{\Omega}^{c}\right)^{3}, \operatorname{div}\left(\varphi \phi_{n}+\mathfrak{D}_{n}\right)=0$ for $n \in \mathbb{N}$. In addition, since $\left\|\mathfrak{D}-\mathfrak{D}_{n}\right\|_{2} \leq \mathfrak{C}\left\|\nabla \varphi \cdot\left(G-\phi_{n}\right)\right\|_{2}$ by Theorem 3.7, we obtain $\left\|\varphi G+\mathfrak{D}-\left(\varphi \phi_{n}+\mathfrak{D}_{n}\right)\right\|_{2} \rightarrow 0$. Moreover, observing that $\operatorname{supp}\left(\varphi G+\mathfrak{D}-\left(\varphi \phi_{n}+\mathfrak{D}_{n}\right) \subset B_{S+2}\right.$, we get

$$
\left|\int_{\bar{\Omega}^{c}}\left(\varphi G+\mathfrak{D}-\left(\varphi \phi_{n}+\mathfrak{D}_{n}\right)\right) \cdot \gamma d x\right| \leq \mathfrak{C}\left\|\varphi G+\mathfrak{D}-\left(\varphi \phi_{n}+\mathfrak{D}_{n}\right)\right\|_{2}\|\nabla \gamma\|_{2}
$$

for $\gamma \in C_{0}^{\infty}\left(\bar{\Omega}^{c}\right)^{3}$, where we applied Poincaré's inequality to $\gamma \mid \Omega_{S+2}$. Since the term $\| \varphi G+$ $\mathfrak{D}-\left(\varphi \phi_{n}+\mathfrak{D}_{n}\right) \|_{2}$ tends to zero (see above), it follows that $\left\|\varphi G+\mathfrak{D}-\left(\varphi \phi_{n}+\mathfrak{D}_{n}\right)\right\|_{-1,2} \rightarrow 0$.

Let $\widetilde{G}$ denote the zero extension of $G$ to $\mathbb{R}^{3}$. Note that $(1-\varphi) \widetilde{G} \mid B_{S+5 / 4}=0$. Reasoning as above, we find that $\left\|(1-\varphi) \widetilde{G}-\mathfrak{D}-\left((1-\varphi) \phi_{n}-\mathfrak{D}_{n}\right)\right\|_{2} \rightarrow 0$, so $(1-\varphi) \widetilde{G}-\mathfrak{D} \in$ $H_{2}\left(\mathbb{R}^{3}\right)^{3}$. Take $\gamma \in C^{\infty}\left(\mathbb{R}^{3}\right)^{3}$. Then $(1-\varphi) \gamma \in C_{0}^{\infty}\left(\bar{\Omega}^{c}\right)^{3}$. Moreover, using (3.1), we get 
$\left\|\gamma\left|B\left\|_{2} \leq \mathfrak{C}\right\| \gamma\right| B\right\|_{6} \leq \mathfrak{C}\left\|\gamma \mid B_{S+1}^{c}\right\|_{6} \leq \mathfrak{C}\|\nabla \gamma\|_{2}$. Therefore $\|\nabla(1-\varphi) \cdot \gamma\|_{2} \leq \mathfrak{C}\|\nabla \gamma\|_{2}$, so we may conclude that $\left|\int_{\mathbb{R}^{3}}(1-\varphi) \widetilde{G} \cdot \gamma d x\right| \leq\|G\|_{-1,2}\|\nabla((1-\varphi) \gamma)\|_{2} \leq \mathfrak{C}\|G\|_{-1,2}\|\nabla \gamma\|_{2}$. Similarly, $\left|\int_{\mathbb{R}^{3}} \mathfrak{D} \cdot \gamma d x\right| \leq\|\mathfrak{D}\|_{2}\|\gamma \mid B\|_{2} \leq \mathfrak{C}\|\mathfrak{D}\|_{2} \cdot\|\nabla \gamma\|_{2}$. Thus we see that $(1-\varphi) \widetilde{G}-$ $\mathfrak{D} \in \widetilde{\mathcal{D}}_{0}^{-1,2}\left(\mathbb{R}^{3}\right)^{3}$. Therefore by Theorem 3.10 , there is a sequence $\left(\psi_{n}\right)$ in $C_{0}^{\infty}\left(\mathbb{R}^{3}\right)^{3}$ with $\operatorname{div} \psi_{n}=0$ for $n \in \mathbb{N}$ and

$$
\left\|(1-\varphi) \widetilde{G}-\mathfrak{D}-\psi_{n}\right\|_{2} \rightarrow 0, \quad\left\|(1-\varphi) \widetilde{G}-\mathfrak{D}-\psi_{n}\right\|_{-1,2} \rightarrow 0 .
$$

Choose $\widetilde{\varphi} \in C^{\infty}\left(\mathbb{R}^{3}\right)$ with $\varphi\left|B_{S+3 / 4}^{c}=1, \varphi\right| B_{S+1 / 4}=0, \quad 0 \leq \widetilde{\varphi} \leq 1$. Recalling that $\operatorname{supp}(1-\varphi) \cup \operatorname{supp}(\mathfrak{D}) \subset B_{S+1}^{c}$, we obtain $(1-\varphi) G-\mathfrak{D}=\widetilde{\varphi}((1-\varphi) G-\mathfrak{D})$. Obviously $\int_{B_{S+1} \backslash \overline{B_{S}}} \nabla \widetilde{\varphi} \cdot \psi_{n} d x=0$, so the function $\widetilde{\mathfrak{D}}_{n}:=\mathfrak{D}(2,0, S, S+1)\left(-\nabla \widetilde{\varphi} \cdot \psi \mid B_{S+1} \backslash \overline{B_{S}}\right)$ for $n \in$ $\mathbb{N}$, is well defined; see Theorem 3.7. This theorem and the relations $\bar{\Omega} \subset B_{S}, \widetilde{\varphi} \mid B_{S+1 / 4}=0$ imply $\widetilde{\varphi} \psi_{n}+\widetilde{\mathfrak{D}}_{n} \in C_{0}^{\infty}\left(\bar{\Omega}^{c}\right)^{3}$ and $\operatorname{div}\left(\widetilde{\varphi} \psi_{n}+\widetilde{D}_{n}\right)=0$ for $n \in \mathbb{N}$, as well as $\left\|\widetilde{\mathfrak{D}}_{n}\right\|_{2} \leq$ $\mathfrak{C}\left\|\nabla \widetilde{\varphi} \cdot \psi_{n}\right\|_{2} \leq \mathfrak{C}\left\|\psi_{n} \mid \Omega_{S+1}\right\|_{2}$. Since $(1-\varphi) G-\mathfrak{D} \mid \Omega_{S+1}=0$, we may conclude from the preceding inequality that

$$
\left\|\widetilde{\mathfrak{D}}_{n}\right\|_{2} \leq \mathfrak{C} \cdot\left\|(1-\varphi) G-\mathfrak{D}-\psi_{n} \mid \Omega_{S+1}\right\|_{2} \leq\left\|(1-\varphi) G-\mathfrak{D}-\psi_{n}\right\|_{2} .
$$

Now we find with the relation $(1-\varphi) G-\mathfrak{D}=\widetilde{\varphi}((1-\varphi) G-\mathfrak{D})$ that $\|(1-\varphi) G-\mathfrak{D}-$ $\left(\widetilde{\varphi} \psi_{n}+\widetilde{\mathfrak{D}}_{n}\right)\left\|_{2} \leq\right\| \widetilde{\varphi}\left((1-\varphi) G-\mathfrak{D}-\psi_{n}\right)\left\|_{2}+\right\| \widetilde{\mathfrak{D}}_{n}\left\|_{2} \leq \mathfrak{C}\right\|(1-\varphi) G-\mathfrak{D}-\psi_{n} \|_{2}$. Therefore from (3.11), $\left\|(1-\varphi) G-\mathfrak{D}-\left(\widetilde{\varphi} \psi_{n}+\widetilde{\mathfrak{D}}_{n}\right)\right\|_{2} \rightarrow 0$.

This leaves us to prove convergence of the sequence $\left(\widetilde{\varphi} \psi_{n}+\widetilde{\mathfrak{D}}_{n} \mid \bar{\Omega}^{c}\right)$ with respect to the norm \|\|$_{-1,2}$. To this end, take $n \in \mathbb{N}$ and $\gamma \in C_{0}^{\infty}\left(\bar{\Omega}^{c}\right)^{3}$. Then, with the relation $(1-\varphi) G-\mathfrak{D}=\widetilde{\varphi}((1-\varphi) G-\mathfrak{D})$,

$$
\begin{aligned}
& \mathfrak{A}_{n}:=\left|\int_{\bar{\Omega}^{c}}\left((1-\varphi) G-\mathfrak{D}-\left(\widetilde{\varphi} \psi_{n}+\widetilde{\mathfrak{D}}_{n}\right)\right) \cdot \gamma d x\right| \\
& \leq\left|\int_{\mathbb{R}^{3}} \widetilde{\varphi}\left((1-\varphi) \widetilde{G}-\mathfrak{D}-\psi_{n}\right) \cdot \gamma d x\right|+\left\|\widetilde{\mathfrak{D}}_{n}\right\|_{2}\left\|\gamma \mid B_{S+1} \backslash \overline{B_{S}}\right\|_{2} \\
& \leq\left\|(1-\varphi) \widetilde{G}-\mathfrak{D}-\psi_{n}\right\|_{-1,2}\|\nabla(\widetilde{\varphi} \gamma)\|_{2}+\mathfrak{C}\left\|(1-\varphi) G-\mathfrak{D}-\psi_{n}\right\|_{2}\left\|\gamma \mid B_{S+1} \backslash \overline{B_{S}}\right\|_{2},
\end{aligned}
$$

where we used (3.12) in the last inequality. But $\|\nabla(\widetilde{\varphi} \gamma)\|_{2} \leq \mathfrak{C}\left(\left\|\gamma \mid B_{S+1} \backslash \overline{B_{S}}\right\|_{2}+\|\nabla \gamma\|_{2}\right)$ and $\left\|\gamma\left|B_{S+1} \backslash \overline{B_{S}}\left\|_{2} \leq \mathfrak{C}\right\| \gamma\right| B_{S+1} \backslash \overline{B_{S}}\right\|_{6} \leq \mathfrak{C}\left\|\gamma \mid{\overline{B_{S}}}^{c}\right\|_{6} \leq \mathfrak{C}\|\nabla \gamma\|_{2}$, with the last inequality following from (3.1). This estimate allows us to deduce from (3.13) that the quantity $\mathfrak{A}_{n}$ is bounded by $\mathfrak{C}\left(\left\|(1-\varphi) \widetilde{G}-\mathfrak{D}-\psi_{n}\right\|_{-1,2}+\left\|(1-\varphi) \widetilde{G}-\mathfrak{D}-\psi_{n}\right\|_{2}\right)\|\nabla \gamma\|_{2}$. Now we may conclude from $(3.11)$ that $\left\|(1-\varphi) G-\mathfrak{D}-\left(\widetilde{\varphi} \psi_{n}+\widetilde{\mathfrak{D}}_{n}\right)\right\|_{-1,2} \rightarrow 0$. In view of the fact that the terms $\left\|\varphi G+\mathfrak{D}-\left(\varphi \phi_{n}+\mathfrak{D}_{n}\right)\right\|_{2},\left\|\varphi G+\mathfrak{D}-\left(\varphi \phi_{n}+\mathfrak{D}_{n}\right)\right\|_{-1,2}$ and $\left\|(1-\varphi) G-\mathfrak{D}-\left(\widetilde{\varphi} \psi_{n}+\widetilde{\mathfrak{D}}_{n}\right)\right\|_{2}$ tend to zero for $n \rightarrow \infty$, as previously shown, the theorem is proved.

Corollary 3.5 Let $G \in L^{2}\left(\bar{\Omega}^{c}\right) \cap \mathfrak{D}_{0}^{-1,2}\left(\bar{\Omega}^{c}\right)$. Then there is a sequence $\left(\phi_{n}\right)$ in $C_{0}^{\infty}\left(\bar{\Omega}^{c}\right)$ such that $\left\|G-\phi_{n}\right\|_{2} \rightarrow 0$ and $\left\|G-\phi_{n}\right\|_{-1,2} \rightarrow 0$.

Proof: This corollary may be proved by the same type of argument as Theorem 3.11, this time based on Corollary 3.4 instead of Theorem 3.10. But the details are considerably less involved. 
In the rest of this section, we deal with the Helmholtz projection, starting with the whole space case. The special feature of that case is that the projection in question applied to a smooth function is given in an explicit way.

$\langle$ theoremT3.150〉

Theorem 3.12 Let $q \in(1, \infty)$. Then there are linear operators $P_{q}: L^{q}\left(\mathbb{R}^{3}\right)^{3} \mapsto H_{q}\left(\mathbb{R}^{3}\right)$ and $Q_{q}: L^{q}\left(\mathbb{R}^{3}\right) \mapsto W_{l o c}^{1, q}\left(\mathbb{R}^{3}\right)$ such that $P_{q}^{2}=P_{q}, G=P_{q}(G)+\nabla Q_{q}(G),\left\|P_{q}(G)\right\|_{q}+$ $\left\|\nabla Q_{q}(G)\right\|_{q} \leq C(q)\|G\|_{q}$ (in particular $\nabla Q_{q}(G) \in L^{q}\left(\mathbb{R}^{3}\right)^{3}$ and $P_{q}\left(\nabla Q_{q}(G)\right)=0$ ) for $G \in L^{q}\left(\mathbb{R}^{3}\right)^{3}$, and $Q_{q}(G)=N * \operatorname{div} G, P_{q}(G)=G-\left(\left(\partial_{l} N\right) * \operatorname{div} G\right)_{1 \leq l \leq 3}$ for $G \in C_{0}^{\infty}\left(\mathbb{R}^{3}\right)^{3}$.

Proof: See [24, Section III.1, in particular p. 147-148 and Theorem III.1.2].

corollaryC3.50) Corollary 3.6 Let $p, q \in(1, \infty)$ and $G \in L^{p}\left(\mathbb{R}^{3}\right)^{3} \cap L^{q}\left(\mathbb{R}^{3}\right)^{3}$. Then $P_{p}(G)=P_{q}(G)$.

Proof: See [12, Theorem 5] or use Lemma 3.1 and Theorem 3.12.

As a supplement to Theorem 2.1, we state some additional properties of the Helmholtz projection on $L^{p}\left(\bar{\Omega}^{c}\right)^{3}$.

corollaryC3.60)

Corollary 3.7 Let $p, q \in(1, \infty)$. Then $\mathcal{P}_{p}(G)=\mathcal{P}_{q}(G)$ for $G \in L^{p}\left(\bar{\Omega}^{c}\right)^{3} \cap L^{q}\left(\bar{\Omega}^{c}\right)^{3}$. Moreover $\left(\mathcal{P}_{p}\right)^{\prime}=\mathcal{P}_{p^{\prime}}$ and $\mathcal{P}_{p}(\nabla \Pi)=0$ for $\Pi \in W_{\text {loc }}^{1, p}\left(\bar{\Omega}^{c}\right)$ with $\nabla \Pi \in L^{p}\left(\bar{\Omega}^{c}\right)^{3}$.

Proof: The first claim of the corollary follows from Lemma 3.1, (2.1) and the last equation in Theorem 2.1. Concerning the proof of the second statement, let $G \in L^{p}\left(\bar{\Omega}^{c}\right)^{3}, H \in$ $L^{p^{\prime}}\left(\bar{\Omega}^{c}\right)^{3}$. We may choose a sequence $\left(\phi_{n}\right)$ in $C_{0}^{\infty}\left(\bar{\Omega}^{c}\right)^{3}$ with $\operatorname{div} \phi_{n}=0$ for $n \in \mathbb{N}$ and $\left\|\phi_{n}-\mathcal{P}_{p^{\prime}}(H)\right\|_{p^{\prime}} \rightarrow 0$. Then, since $\nabla \mathcal{Q}_{p}(G) \in L^{p}\left(\bar{\Omega}^{c}\right)^{3}$ (Theorem 2.1), we have $\int_{\bar{\Omega}^{c}} \nabla \mathcal{Q}_{p}(G)$. $\mathcal{P}_{p^{\prime}}(H) d x=\lim _{n \rightarrow \infty} \iint_{\bar{\Omega}^{c}} \nabla \mathcal{Q}_{p}(G) \cdot \phi_{n} d x=0$, so $\int_{\bar{\Omega}^{c}} G \cdot \mathcal{P}_{p^{\prime}}(H) d x=\iint_{\bar{\Omega}^{c}} \mathcal{P}_{p}(G) \cdot \mathcal{P}_{p^{\prime}}(H) d x$ by Theorem 2.1. An analogous equation holds with $\mathcal{P}_{p}(G) \cdot H$ in the place of $G \cdot \mathcal{P}_{p^{\prime}}(H)$ on the left-hand side. The second claim of the theorem follows. The third follows from the second by the same type of argument.

$\left\langle\right.$ theoremT3.160〉 Theorem 3.13 Let $G \in \mathcal{D}_{0}^{-1,2}\left(\bar{\Omega}^{c}\right)^{3} \cap L^{2}\left(\bar{\Omega}^{c}\right)^{3}$. Then $\mathcal{P}_{2}(G) \in \mathcal{D}_{0}^{-1,2}\left(\bar{\Omega}^{c}\right)^{3} \cap H_{2}\left(\bar{\Omega}^{c}\right)$ and $\left\|\mathcal{P}_{2}(G)\right\|_{-1,2} \leq \mathfrak{C}\|G\|_{*}$.

Proof: Obviously we have $\mathcal{P}_{2}(G) \in H_{2}\left(\bar{\Omega}^{c}\right)$ (Theorem 2.1). Let $\gamma \in C_{0}^{\infty}\left(\bar{\Omega}^{c}\right)^{3}$. We are going to show that $\left|\int_{\bar{\Omega}^{c}} \mathcal{P}_{2}(G) \cdot \gamma d x\right| \leq \mathfrak{C}\|G\|_{*}\|\nabla \gamma\|_{2}$. This proves Theorem 3.13; see Theorem 3.6. In order to establish the preceding estimate, we construct functions $\gamma^{(1)} \in$ $C^{\infty}\left(\mathbb{R}^{3}\right)^{3}, \gamma^{(2)} \in C_{0}^{\infty}\left(B_{S+1}\right)^{3}$ with $\gamma^{(1)} \mid \bar{\Omega}^{c} \in W_{0}^{1,2}\left(\bar{\Omega}^{c}\right)^{3} \cap H_{2}\left(\bar{\Omega}^{c}\right),\left\|\nabla \gamma^{(i)}\right\|_{2} \leq \mathfrak{C}\|\nabla \gamma\|_{2}$ for $i \in\{1,2\}$, and $\int \bar{\Omega}^{c} \mathcal{P}_{2}(G) \cdot \gamma d x=\int \bar{\Omega}^{c} \mathcal{P}_{2}(G) \cdot\left(\gamma^{(1)}+\gamma^{(2)}\right) d x$. These relations will yield the estimate we look for. We start by setting $\mathfrak{N}:=\left(\sum_{l=1}^{3} \partial_{j} \partial_{l}\left(N * \gamma_{l}\right)\right)_{1 \leq j \leq 3}$. Then $\mathfrak{N} \in C^{\infty}\left(\mathbb{R}^{3}\right)^{3} \cap L^{2}\left(\mathbb{R}^{3}\right)^{3}$ according to (3.3) and (3.4). Moreover, again referring to (3.3), we note that $\partial_{m} \mathfrak{N}_{j}=\partial_{m} \partial_{j}(N * \operatorname{div} \gamma)$ for $1 \leq j, m \leq 3$, so $\nabla \mathfrak{N}_{j} \in L^{2}\left(\mathbb{R}^{3}\right)^{3}$ and $\left\|\nabla \mathfrak{N}_{j}\right\|_{2} \leq C\|\operatorname{div} \gamma\|_{2} \leq C\|\nabla \gamma\|_{2}$ for $1 \leq j \leq 3$ by (3.4). It further follows with (3.3) that $\operatorname{div} \mathfrak{N}=\Delta(N * \operatorname{div} \gamma)=\operatorname{div} \gamma$. Thus we have found that

$$
\gamma-\mathfrak{N} \in C^{\infty}\left(\mathbb{R}^{3}\right)^{3} \cap W^{1,2}\left(\mathbb{R}^{3}\right)^{3}, \quad \operatorname{div}(\gamma-\mathfrak{N})=0, \quad\|\nabla(\gamma-\mathfrak{N})\|_{2} \leq C\|\nabla \gamma\|_{2} . \quad(3.14) \text { T3.160.30 }
$$

Put $B:=B_{S+1} \backslash \overline{B_{S}}$, and take $\varphi \in C^{\infty}\left(\mathbb{R}^{3}\right)$ with $0 \leq \varphi \leq 1, \varphi\left|B_{S+1 / 4}=0, \varphi\right| B_{S+3 / 4}^{c}=1$. Then $\nabla \varphi \cdot(\gamma-\mathfrak{N}) \in C^{\infty}(B)$ and $\int_{B} \nabla \varphi \cdot(\gamma-\mathfrak{N}) d x=0$, so we may consider $\mathfrak{D}:=$ $\mathfrak{D}(2,0, S, S+1)(-\nabla \varphi \cdot(\gamma-\mathfrak{N}) \mid B)$; see Theorem 3.7. Note that by that latter theorem, 
we have $\mathfrak{D} \in C_{0}^{\infty}(B)^{3}$ and

$$
\|\mathfrak{D}\|_{1,2} \leq \mathfrak{C}\|\nabla \varphi \cdot(\gamma-\mathfrak{N})\|_{2} \leq \mathfrak{C}\left\|\gamma-\mathfrak{N}\left|B\left\|_{6} \leq \mathfrak{C}\right\| \gamma-\mathfrak{N}\right| B_{S}^{c}\right\|_{6} \leq \mathfrak{C}\|\nabla(\gamma-\mathfrak{N})\|_{2},(3.15) \text { T3.160.40 }
$$

where the last inequality follows from (3.1). Now put $\gamma^{(1)}:=\varphi(\gamma-\mathfrak{N})+\mathfrak{D}$. Then $\gamma^{(1)} \in$ $C^{\infty}\left(\mathbb{R}^{3}\right)^{3} \cap W^{1,2}\left(\mathbb{R}^{3}\right)^{3}, \gamma^{(1)} \mid B_{S}=0, \operatorname{div} \gamma^{(1)}=0$, in particular $\gamma^{(1)} \mid \bar{\Omega}^{c} \in W_{0}^{1,2}\left(\bar{\Omega}^{c}\right)^{3}$. Due to (3.14) and (3.15), we have $\left\|\nabla \gamma^{(1)}\right\|_{2} \leq \mathfrak{C}\|\nabla(\gamma-\mathfrak{N})\|_{2} \leq \mathfrak{C}\|\nabla \gamma\|_{2}$, where we used that (3.15) yields an estimate of $\|\mathfrak{D}\|_{2}$ as well as of $\|\nabla \varphi \cdot(\gamma-\mathfrak{N})\|_{2}$ against $\mathfrak{C} \cdot\|\nabla(\gamma-\mathfrak{N})\|_{2}$. Put $\gamma^{(2)}:=(1-\varphi)(\gamma-\mathfrak{N})-\mathfrak{D}=\gamma-\mathfrak{N}-\gamma^{(1)}$. Then $\gamma^{(2)} \in C_{0}^{\infty}\left(B_{S+1}\right)^{3}$, so we get by Poincaré's inequality that $\left\|\gamma^{(2)}\right\| \leq \mathfrak{C}\left\|\nabla \gamma^{(2)}\right\|_{2}$. Hence by the inequality $\left\|\nabla \gamma^{(1)}\right\| \leq \mathfrak{C}\|\nabla \gamma\|_{2}$ shown above and by (3.14), the estimate $\left\|\gamma^{(2)}\right\| \leq \mathfrak{C}\left\|\nabla\left(\gamma-\mathfrak{N}-\gamma^{(1)}\right)\right\|_{2} \leq \mathfrak{C}\|\nabla \gamma\|_{2}$ holds. We recall that $\mathfrak{N} \in L^{2}\left(\mathbb{R}^{3}\right)^{3}$. Moreover (3.3) yields $\mathfrak{N}=\nabla(N * \operatorname{div} \gamma)$ and $N * \operatorname{div} \gamma \in C^{\infty}\left(\mathbb{R}^{3}\right)$, so by the second and third equation in Corollary 3.7, $\int_{\bar{\Omega}^{c}} \mathcal{P}_{2}(G) \cdot \mathfrak{N} d x=\int \bar{\Omega}^{c} G \cdot \mathcal{P}_{2}(\mathfrak{N}) d x=0$. Now we get

$$
\int_{\bar{\Omega}^{c}} \mathcal{P}_{2}(G) \cdot \gamma d x=\int_{\bar{\Omega}^{c}} \mathcal{P}_{2}(G) \cdot(\gamma-\mathfrak{N}) d x=\int_{\bar{\Omega}^{c}} \mathcal{P}_{2}(G) \cdot\left(\gamma^{(1)}+\gamma^{(2)}\right) d x .
$$

But $\gamma^{(1)} \mid \bar{\Omega}^{c} \in W_{0}^{1,2}\left(\bar{\Omega}^{c}\right)^{3}$ and $\operatorname{div} \gamma^{(1)}=0$, as mentioned above, so $\gamma^{(1)} \mid \bar{\Omega}^{c} \in H_{2}\left(\bar{\Omega}^{c}\right)^{3}$ by Theorem 3.2. Thus $\int_{\bar{\Omega}^{c}} \mathcal{P}_{2}(G) \cdot \gamma^{(1)} d x=\int_{\bar{\Omega}^{c}} G \cdot \gamma^{(1)} d x$. Now we may conclude from the relations $\left\|\nabla \gamma^{(1)}\right\|_{2} \leq \mathfrak{C}\|\nabla \gamma\|_{2}$ and $\left\|\gamma^{(2)}\right\|_{2} \leq \mathfrak{C}\|\nabla \gamma\|_{2}$ established previously, and from (3.16) that $\left|\int_{\bar{\Omega}^{c}} \mathcal{P}_{2}(G) \cdot \gamma d x\right| \leq\|G\|_{-1,2}\left\|\nabla \gamma^{(1)}\right\|_{2}+\left\|\mathcal{P}_{2}(G)\right\|_{2}\left\|\gamma^{(2)}\right\|_{2} \leq \mathfrak{C}\left(\|G\|_{-1,2}+\right.$ $\left.\|\mathcal{P}(G)\|_{2}\right)\|\nabla \gamma\|_{2}$. The looked-for inequality $\left|\int_{\bar{\Omega}^{c}} \mathcal{P}_{2}(G) \cdot \gamma d x\right| \leq \mathfrak{C}\|G\|_{*}\|\nabla \gamma\|_{2}$ now follows with (2.1).

\section{The Stokes and Oseen system and associated resolvent problems: some known results.}

Our theory relies heavily on existence and regularity results for the Stokes and Oseen system, and on resolvent estimates related to these systems. For the convenience of the reader, we specify in this section what exactly will be relevant in this respect. We begin by considering the Stokes resolvent problem.

$\left\langle\right.$ theoremT4.10〉 Theorem 4.1 Let $p \in(1, \infty), \lambda \in(0, \infty), V \in \mathcal{D}(\mathcal{L})$ (see (1.11)), $\Pi \in W_{\text {loc }}^{1,2}\left(\bar{\Omega}^{c}\right), G \in$ $L^{2}\left(\bar{\Omega}^{c}\right)^{3} \cap L^{p}\left(\bar{\Omega}^{c}\right)^{3}$ with $-\Delta V+\lambda V+\nabla \Pi=G$, div $V=0$. Then $V \in W^{2, p}\left(\bar{\Omega}^{c}\right)^{3}$ and $\|V\|_{2, p} \leq \mathfrak{C}(p)\|G\|_{p}$

Proof: According to [25], [5] or [7], [8], there are functions $W \in W^{2, p}\left(\bar{\Omega}^{c}\right)^{3} \cap W_{0}^{1, p}\left(\bar{\Omega}^{c}\right)^{3} \cap$ $H_{p}\left(\bar{\Omega}^{c}\right), \widetilde{\Pi} \in W_{l o c}^{1, p}\left(\bar{\Omega}^{c}\right)^{3}$ such that $-\Delta W+\lambda W+\nabla \widetilde{\Pi}=G, \operatorname{div} W=0$ and $\|W\|_{2, p} \leq$ $\mathfrak{C}(p)\|G\|_{p}$. Then $V=W$ by [11, Lemma 2.5], so the theorem follows.

Next we observe that for any weak solution of the Oseen or the Oseen resolvent system, there exists an associate pressure.

$\langle$ theoremT4.20〉

Theorem 4.2 Let $A \subset \mathbb{R}^{3}$ be open, $q \in(1, \infty), \lambda \in \mathbb{C}, G \in L_{\text {loc }}^{q}(A), V \in W_{\text {loc }}^{1,1}(A)^{3}$ with $\nabla V \in L_{l o c}^{q}(A)^{9}, \operatorname{div} V=0$,

$$
\int_{A}\left(\nabla V \cdot \nabla \psi+\left(\tau \partial_{1} V+\lambda V-G\right) \cdot \psi\right) d x=0 \quad \text { for } \psi \in C_{0}^{\infty}(A)^{3} \text { with } \operatorname{div} \psi=0 .
$$


Then there is $\Pi \in L_{\text {loc }}^{q}(A)$ ("pressure associated with $V$ ") such that

$$
\left.\int_{A}\left(\nabla V \cdot \nabla \psi+\left(\tau \partial_{1} V+\lambda V-G\right) \cdot \psi\right)-\Pi \operatorname{div} \psi\right) d x=0 \quad \text { for } \psi \in C_{0}^{\infty}(A)^{3} .
$$

Proof: Let $B \subset \mathbb{R}^{3}$ be open and bounded with $\bar{B} \subset A$. Then $-\tau \partial_{1} V-\lambda V+G \mid B \in$ $L^{q}(B)^{3}$ (Theorem 3.1), and Poincaré's inequality yields $\left|\int_{B}\left(-\tau \partial_{1} V-\lambda V+G\right) \cdot \psi d x\right| \leq$ $\mathfrak{C}(\lambda)\left(\left\|V\left|B\left\|_{1, q}+\right\| G\right| B\right\|_{q}\right)\|\psi\|_{q^{\prime}}$ for $\psi \in W_{0}^{1, q^{\prime}}(B)^{3}$. Now Theorem 4.2 follows from [23, Lemma IV.1.1] and (4.1).

$\langle$ theoremT4.30〉

We cite a theorem on interior regularity of solutions to the Stokes system.

Theorem 4.3 ([23, Theorem IV.4.1]) Let $A \subset \mathbb{R}^{3}$ be open, $m \in \mathbb{N}_{0}, r \in(1, \infty), G \in$ $W_{l o c}^{m, r}(A)^{3}, V \in W_{l o c}^{1,1}(A)^{3}$ with $\nabla V \in L_{l o c}^{r}(A)^{9}$, div $V=0$ and

$$
\int_{A}(\nabla V \cdot \nabla \psi-G \cdot \psi) d x=0 \quad \text { for } \psi \in C_{0}^{\infty}(A)^{3} \text { with } \operatorname{div} \psi=0
$$

( $V$ weak solution of the Stokes system). Then $V \in W_{l o c}^{2+m, r}(A)^{3}$. Let $\Pi \in L_{l o c}^{r}(A)$ with

$$
\int_{A}(\nabla V \cdot \nabla \psi-\Pi \operatorname{div} \psi-G \cdot \psi) d x=0 \quad \text { for } \psi \in C_{0}^{\infty}(A)^{3}
$$

(pressure associated with $V)$. Then $\Pi \in W_{l o c}^{1+m, r}(A)$ and $-\Delta V+\nabla \Pi=G$.

The preceding theorem implies interior regularity for solutions of the Oseen resolvent problem (if $\lambda=0$ : Oseen system). For the convenience of the reader, we indicate a proof.

Corollary 4.1 Let $A \subset \mathbb{R}^{3}$ be open, $q, s \in(1, \infty), \lambda \in \mathbb{C}, G \in L_{\text {loc }}^{q}(A)^{3}, V \in W_{\text {loc }}^{1,1}(A)^{3}$ with $\nabla V \in L_{\text {loc }}^{s}(A)^{9}$ and div $V=0$. Suppose that (4.1) holds. Then $V \in W_{l o c}^{2, q}(A)^{3}$. Let $\Pi \in L_{\text {loc }}^{s}(A)$ be a pressure associated with $V$ (Theorem 4.2). Then $\Pi \in W_{l o c}^{1, q}(A)$ and

$$
-\Delta V+\tau \partial_{1} V+\lambda V+\nabla \Pi=G, \quad \operatorname{div} V=0 .
$$

Proof: Theorem 3.1 yields $V \in W_{l o c}^{1, s}(A)^{3}$. Abbreviate $H:=-\tau \partial_{1} V-\lambda V+G$. Put $r_{1}:=\min \{q, s\}$. Then $H$ and $\nabla V$ are $L_{l o c}^{r_{1}}$-functions in $A$, so Theorem 4.3 implies that $V \in W_{l o c}^{2, r_{1}}(A)^{3}, \Pi \in W_{l o c}^{1, r_{1}}(A)$ and (4.5) holds. If $q \leq s$, Corollary 4.1 is proved. Else we apply a Sobolev inequality to obtain $V \in W_{l o c}^{1,3 / 2}(A)^{3}$. Put $r_{2}:=\min \{q, 3 / 2\}$. Then we may conclude that $\nabla V$ and $H$ are $L_{l o c}^{r_{2}}$-functions in $A$, so $V \in W_{l o c}^{2, r_{2}}(A)^{3}$ and $\Pi \in W_{l o c}^{1, r_{2}}(A)$ by Theorem 4.3. Thus we are done if $q \leq 3 / 2$, otherwise $V \in W_{l o c}^{2,3 / 2}(A)^{3} \subset W_{l o c}^{1,3}(A)^{3}$. Setting $r_{3}:=\min \{q, 3\}$, we thus have $\partial_{l} V, H \in L_{l o c}^{r_{3}}(A)^{3}(1 \leq l \leq 3)$. Another reference to Theorem 4.3 yields $V \in W_{l o c}^{r_{3}, 2}(A)^{3}$ and $\Pi \in W_{l o c}^{1, r_{3}}(A)$. This settles the case $q \leq 3$. Else $V \in W_{l o c}^{3,2}(A)^{3} \subset W_{l o c}^{1, q}(A)^{3}$, hence $H \in L_{l o c}^{q}(A)^{3}$, so Corollary 4.1 follows by once more referring to Theorem 4.3 .

Concerning regularity near the boundary, in many situations we again rely on a result about Stokes flows: 
Theorem 4.4 ([24, Lemma IV.6.1]) Let $A \subset \mathbb{R}^{3}$ be open and bounded, with $C^{2}$-boundary, $r \in(1, \infty), G \in L^{r}(A)^{3}, V \in W_{\text {loc }}^{1,1}(A)^{3}$ with $\nabla V \in L^{r}(A)^{9}$ (hence $V \in W^{1, r}(A)^{3}$ by Theorem 3.1), $V \mid \partial A \in W^{2-1 / r, r}(\partial A)^{3}$, div $V=0$, and with $V$ satifying (4.3) (weak solution of the Stokes system). Then $V \in W^{2, r}(A)^{3}$. Let $\Pi \in L_{\text {loc }}^{r}(A)$ be such that (4.4) is satisfied (associated pressure). Then $\Pi \in W^{1, r}(A)$ and $-\Delta V+\nabla \Pi=G$.

corollaryC4.20>

Corollary 4.2 Let $A \subset \mathbb{R}^{3}$ be open and bounded, with $C^{2}$-boundary, $s, q \in(1, \infty), \lambda \in$ $\mathbb{C}, G \in L^{q}(A)^{3}, V \in W_{\text {loc }}^{1,1}(A)^{3}$ with $\nabla V \in L^{s}(A)^{9}$ (hence $V \in W^{1, s}(A)^{3}$ by Theorem 3.1), $V \mid \partial A \in W^{2-1 / q, q}(\partial A)^{3}$, div $V=0$, and with $V$ satifying (4.1) ( $V$ weak solution of (4.5)). Then $V \in W^{2, q}(A)^{3}$. Let $\Pi \in L_{\text {loc }}^{s}(A)$ be a pressure associated with $V$ (Theorem 4.2). Then $\Pi \in W^{1, q}(A)$ and equation (4.5) holds.

Proof: We have $V \mid \partial A \in W^{2-1 / r, r}(\partial A)^{3}$ for $r \in[1, q]$. Thus we may proceed in the same iterative way as in the proof of Corollary 4.1, but with the references to Theorem 4.3 replaced by ones to Theorem 4.4.

Corollary 4.3 Let $s, q \in(1, \infty), \lambda \in \mathbb{C}, G \in L_{l o c}^{q}\left(\mathbb{R}^{3}\right)^{3}, V \in W_{\text {loc }}^{1,1}\left(\bar{\Omega}^{c}\right)^{3}$ with $\nabla V \mid \Omega_{R} \in$ $L^{s}\left(\Omega_{R}\right)^{9}$ (hence $V \mid \Omega_{R} \in W^{1, s}\left(\Omega_{R}\right)^{3}$ by Theorem 3.1) for any $R \in(0, \infty)$ with $\bar{\Omega} \subset$ $B_{R}, V \mid \partial \Omega \in W^{2-1 / q, q}(\partial \Omega)^{3}$, div $V=0$. Further suppose that $V$ satifies (4.1) with $A=\bar{\Omega}^{c}$. Then $V \mid \Omega_{R} \in W^{2, q}\left(\Omega_{R}\right)^{3}$ for $R$ as above. Let $\Pi \in L_{\text {loc }}^{s}\left(\bar{\Omega}^{c}\right)$ be a pressure associated with $V$ (Theorem 4.2). Then $\Pi \mid \Omega_{R} \in W^{1, q}\left(\Omega_{R}\right)$ for $R$ as above, and equation (4.5) holds.

Proof: Corollary 4.1 yields $V \in W_{l o c}^{2, q}\left(\bar{\Omega}^{c}\right)^{3}, \Pi \in W_{l o c}^{1, q}\left(\bar{\Omega}^{c}\right)$ as well as (4.5). Let $R \in(0, \infty)$ with $\bar{\Omega} \subset B_{R}$. Since $V \in W_{l o c}^{2, q}\left(\bar{\Omega}^{c}\right)^{3}$, we have $V \mid \partial B_{R} \in W^{2-1 / q, q}\left(\partial B_{R}\right)^{3}$. Thus, recalling the assumption on $V \mid \partial \Omega$, we obtain $V \mid \partial \Omega_{R} \in W^{2-1 / q, q}\left(\partial \Omega_{R}\right)^{3}$. Now Corollary 4.2 yields $V\left|\Omega_{R} \in W^{2, q}\left(\Omega_{R}\right)^{3}, \Pi\right| \Omega_{R} \in W^{1, q}\left(\Omega_{R}\right)$.

Next we present a criterion on $C^{\infty}$-regularity.

corollaryc4.40〉 Corollary 4.4 Let $A \subset \mathbb{R}^{3}$ be open, $q \in(1, \infty), \lambda \in \mathbb{C}, G \in C^{\infty}(A)^{3}, V \in W_{\text {loc }}^{1,1}(A)^{3}$ with $\nabla V \in L^{q}(A)^{9}$, div $V=0$, and with $V$ satisfying (4.1). Let $\Pi \in L_{\text {loc }}^{q}(A)$ be a pressure associated with $V$ (Theorem 4.2). Then $V \in C^{\infty}(A)^{3}, \Pi \in C^{\infty}(A)$ and equation (4.5) holds.

Proof: In the case $\lambda=0$, we may refer to [23, Theorem VII.1.1]. But both the case $\lambda=0$ and $\lambda \neq 0$ may be reduced to Theorem 4.3. In fact, by Corollary 4.1, we have $V \in W_{l o c}^{2, q}(A)^{3}, \Pi \in W_{l o c}^{1, q}(A)$, and equation (4.5) holds. Suppose that $n \in \mathbb{N}_{0}$ and $V \in W_{l o c}^{2+n, q}(A)^{3}, \Pi \in W_{l o c}^{1+n, q}(A)$. Then $-\tau \partial_{1} V-\lambda V+G \in W_{l o c}^{1+n, q}(A)^{3}$, so Theorem 4.3 implies $V \in W_{l o c}^{3+n, q}(A)^{3}$ and $\Pi \in W_{l o c}^{2+n, q}(A)$. Therefore it follows by induction that $V \in W_{l o c}^{m, q}(A)^{3}$ and $\Pi \in W_{l o c}^{m-1, q}(A)$ for any $m \in \mathbb{N}, m \geq 2$. Corollary 4.4 follows by applying a Sobolev inequality to $V \mid B_{\epsilon}(x)$ and $\Pi \mid B_{\epsilon}(x)$, where $x$ is an arbitrary point in $A$ and $\epsilon>0$ is chosen in such a way that $\overline{B_{\epsilon}(x)} \subset A$.

We cite an existence result for weak solutions of the Oseen system $((4.5)$ with $\lambda=0)$.

$\langle$ theoremT4.50〉

Theorem 4.5 ([1, Proposition 4.2]) Let $A \subset \mathbb{R}^{3}$ be open and bounded, with $C^{2}$-boundary, $p \in(1, \infty), G \in W_{0}^{-1, p}(A)^{3}$. Then there is a unique function $V \in W_{0}^{1, p}(A)^{3}$ and 
a function $\Pi \in L^{q}(A)$, unique up to a constant, such that divu $=0$, and such that (4.2) holds with $\lambda=0$ (Oseen system) and $\int_{A} G \cdot \psi d x$ replaced by $G(\psi)$.

We are going to exploit this theorem in order to obtain weak solutions of the resolvent problem (4.5). These weak solutions have the special feature that the pressure belongs to a uniqueness class that will be convenient in what follows. A proof of this result is well known in principle: it makes use of the fact that on bounded domains, the resolvent term $\lambda V$ may be considered as a compact perturbation of the Laplace operator. However, since some details are perhaps less evident, and for the convenience of the reader, we indicate the main elements of this proof.

Corollary 4.5 Let $A \subset \mathbb{R}^{3}$ be open and bounded, with $C^{2}$-boundary. Let $\zeta \in C_{0}^{\infty}(A)$ with $\zeta \geq 0, \int_{A} \zeta d x>0$. Let $p \in(1, \infty), \lambda \in K_{\tau}, G \in W_{0}^{-1, p}(A)^{3}$. Then there is a unique pair of functions $(V, \Pi) \in W_{0}^{1, p}(A)^{3} \times L^{p}(A)$ such that $\int_{A} \zeta \Pi d x=0$, div $V=0$, and such that (4.2) holds with $\int_{A} G \cdot \psi d x$ replaced by $G(\psi)$. Moreover $\|V\|_{1, p}+\|\Pi\|_{p} \leq \mathfrak{C}(p, \lambda)\|G\|_{-1, p}$. If $p<3, s \in[p, 3 p /(3-p)]$, we additionally have $\|V\|_{s} \leq \mathfrak{C}(p, \lambda)\|G\|_{-1, p}$.

Proof: Put $W_{0, \sigma}^{1, p}:=\left\{W \in W_{0}^{1, p}(A)^{3}: \operatorname{div} W=0\right\}, L_{\zeta}^{p}:=\left\{\varphi \in L^{p}(A): \int_{A} \varphi \zeta d x=\right.$ $0\}, \mathfrak{W}_{p}:=W_{0, \sigma}^{1, p} \times L_{\zeta}^{p}, \quad\|\mid(V, \Pi)\|:=\|V\|_{1, p}+\|\Pi\|_{p}$ for $(V, \Pi) \in \mathfrak{W}_{p}$. Then $\mathfrak{W}_{p}$ is a vector space, and the mapping $\left\||\||\right.$ is a norm which turns $\mathfrak{W}_{p}$ into a Banach space. Define $\mathfrak{F}: \mathfrak{W}_{p} \mapsto W_{0}^{-1, p}(A)^{3}$ by $\mathfrak{F}(V, \Pi)(\psi):=\int_{A}\left(\nabla V \cdot \nabla \psi+\tau \partial_{1} V \cdot \psi-\Pi \operatorname{div} \psi\right) d x$ for $\psi \in W_{0}^{1, p^{\prime}}(A)^{3},(V, \Pi) \in \mathfrak{W}_{p}$. Obviously the operator $\mathfrak{F}$ is well defined, linear and bounded, and by Theorem 4.5, it is onto. In addition, due to this theorem and because the zero function is the only constant function in $L_{\zeta}^{p}$, this operator is one-to-one. Thus $\mathfrak{F}$ is bijective, so the open mapping theorem implies there is a constant $C_{0}>0$ with $\|V\|_{1, p}+\|\Pi\|_{p} \leq C_{0}\|\mathfrak{F}(V, \Pi)\|_{-1, p}$ for $(V, \Pi) \in \mathfrak{W}_{p}$. For $\varrho \in \mathbb{C}$, define $\mathfrak{K}_{\varrho}: \mathfrak{W}_{p} \mapsto W_{0}^{-1, p}(A)^{3}$ by $\mathfrak{K}_{\varrho}(V, \Pi)(\psi):=\int_{A} \varrho V \cdot \psi d x$ for $(V, \Pi) \in \mathfrak{W}_{p}, \psi \in W_{0}^{1, p^{\prime}}(A)^{3}$. This operator $\mathfrak{K}_{\varrho}$ is linear, bounded and compact. As concerns compactness, we note that the operator $(V, \Pi) \mapsto \varrho V$ from $\mathfrak{W}_{p}$ into $L^{p}(A)^{3}$ is bounded, and then refer to Theorem 3.3. Let $\varrho \in \mathbb{C} \backslash\{0\}$ with $\Re \varrho \geq 0$. If $p \geq 2$, it may be shown by some partial integrations that $\Re\left(\mathfrak{F}+\mathfrak{K}_{\varrho}\right)(V, \Pi)(V)>0$ for $(V, \Pi) \in \mathfrak{W}_{p}$ with $V \neq 0$. Thus we get with Corollary 3.2 that the operator $\mathfrak{F}+\mathfrak{K}_{\varrho}$ is one-to-one if $p \geq 2$. Suppose that $p<2$ and the pair $(V, \Pi) \in \mathfrak{W}_{p}$ satisfies the equation $\left(\mathfrak{F}+\mathfrak{K}_{\varrho}\right)(V, \Pi)=0$. Then Corollary 4.2 yields $V \in W^{2, p^{\prime}}(A)^{3}, \Pi \in W^{1, p^{\prime}}(A)$. Therefore the term $\left(\mathfrak{F}+\mathfrak{K}_{o}\right)(V, \Pi)(V)$ is well defined, and again by partial integration we may conclude this term is strictly positive if $V \neq 0$. As a consequence, the operator $\mathfrak{F}+\mathfrak{K}_{\varrho}$ is one-to-one in the case $p<2$, too. On the other hand, since $\mathfrak{F}$ is linear, bounded and bijective and $\mathfrak{K}_{\varrho}$ is linear and compact, the sum $\mathfrak{F}+\mathfrak{K}_{\varrho}$ is Fredholm with index zero. All these observations taken together imply that $\mathfrak{F}+\mathfrak{K}_{\varrho}$ is bijective, so by the open mapping theorem, there is a constant $C_{\varrho}>0$ with $\|V\|_{1, p}+\|\Pi\|_{p} \leq C_{\varrho}\left\|\left(\mathfrak{F}+\mathfrak{K}_{\varrho}\right)(V, \Pi)\right\|_{-1, p}$ for $(V, \Pi) \in \mathfrak{W}_{p}$. This is true for any $\varrho \in \mathbb{C} \backslash\{0\}$ with $\Re \varrho \geq 0$. Recall that in the case $\varrho=0$, the preceding inequality was proved further above. As a consequence of these estimates, we get for $\varrho, \widetilde{\varrho} \in \mathbb{C}$ with $\Re \varrho \geq 0,(V, \Pi) \in \mathfrak{W}_{p}$ that $\left\|\left(\mathfrak{F}+\mathfrak{K}_{\widetilde{\varrho}}\right)(V, \Pi)\right\|_{-1, p} \geq$ $\left\|\left(\mathfrak{F}+\mathfrak{K}_{\varrho}\right)(V, \Pi)\right\|_{-1, p}-|\varrho-\widetilde{\varrho}|\|V\|_{p} \geq\left(C_{\varrho}^{-1}-|\varrho-\widetilde{\varrho}|\right)\left(\|V\|_{1, p}+\|\Pi\|_{p}\right)$. Thus, for any $\varrho_{0} \in \mathbb{C}$ with $\Re \varrho_{0} \geq$, there is some $\epsilon\left(\varrho_{0}\right)$ such that for any $\varrho \in \mathbb{C}$ with $\left|\varrho-\varrho_{0}\right| \leq \epsilon\left(\varrho_{0}\right)$, we have $\|V\|_{1, p}+\|\Pi\|_{p} \leq 2 C_{\varrho}\left\|\left(\mathfrak{F}+\mathfrak{K}_{\varrho}\right)(V, \Pi)\right\|_{-1, p}$ for $(V, \Pi) \in \mathfrak{W}_{p}$. Now the first estimate at the end of Corollary 4.5 follows by an open covering argument, whereas the second may be 
deduced from the first by a Sobolev inequality.

We turn to strong solutions of (4.5) on bounded domains.

$\langle$ theoremT4.60〉

Theorem 4.6 Let $A \subset \mathbb{R}^{3}$ be open and bounded, with $C^{2}$-boundary, $q \in(1, \infty), \epsilon>$ $0, \lambda \in \mathbb{C}$ with $\Re \lambda \geq 0$ and $|\lambda| \leq \epsilon, G \in L^{p}(A)^{3}$. Then there is a unique pair of functions $(V, \Pi) \in W^{2, q}(A)^{3} \times W^{1, q}(A)$ with $V \in W_{0}^{1, q}(A)^{3}$, div $V=0, \int_{A} \Pi d x=0$, and with $(V, \Pi)$ satisfying (4.5). This pair satifies the estimate $\|V\|_{2, q}+\|\Pi\|_{1, q} \leq \mathfrak{C}(q, \epsilon)\|G\|_{q}$.

Proof: A direct reference is [32, Proposition 2.6], where a much more detailed result is provided. But Theorem 4.6 may also be deduced by starting with an existence and a uniqueness result for strong solutions of the Oseen system on bounded domains (see [1, Proposition 4.3] for example), and then proceed to a perturbation argument as in the proof of Corollary 4.5.

Corollary 4.6 In the situation of the preceding theorem, we have $\|V\|_{p} \leq \mathfrak{C}(p, q, \epsilon)\|G\|_{q}$ for $p \in\left[1,(1 / q-2 / 3)^{-1}\right]$ if $q<3 / 2 ;\|\nabla V\|_{p} \leq \mathfrak{C}(p, q, \epsilon)\|G\|_{q}$ for $p \in\left[1,(1 / q-1 / 3)^{-1}\right]$ if $q<3$, and for $p \in[1, \infty)$ if $q=3$. Moreover, if $\lambda \neq 0, q \leq 2, p \in[q, 2]$, the estimate $\|V\|_{p} \leq \mathfrak{C}(p, q, \epsilon)|\lambda|^{2-4(1-1 / q+1 / p)}\|G\|_{q}$ is valid.

Proof: The corollary follows from Theorem 4.6 and Sobolev estimates. Concerning the last estimate in the corollary, we note that $2-4(1-1 / q+1 / p) \leq 0$ if $q \leq 2, p \in[q, 2]$, so that in the case $\lambda \neq 0$, we have $1 \leq C(p, q, \epsilon)|\lambda|^{2-4(1-1 / q+1 / p)}$.

In the ensuing three theorems, we consider problem (1.18) (Oseen resolvent system in $\bar{\Omega}^{c}$, with Dirichlet boundary conditions), first for $\lambda=0$ (Oseen system), and then for $\lambda \neq 0$.

$\langle$ theoremT4.70〉

Theorem 4.7 ([23, Theorem VII.7.1]) Let $r \in(1,2), G \in L^{r}\left(\bar{\Omega}^{c}\right)^{3}$. Then there are functions $V \in W_{l o c}^{2, r}\left(\bar{\Omega}^{c}\right)^{3} \cap L^{2 r /(2-r)}\left(\bar{\Omega}^{c}\right)^{3}, \Pi \in W_{\text {loc }}^{1, r}\left(\bar{\Omega}^{c}\right)$ such that the relations $\nabla V \in$ $L^{4 r /(4-r)}\left(\bar{\Omega}^{c}\right)^{9}, \partial_{j} V_{k}, \partial_{j} \Pi \in L^{r}\left(\bar{\Omega}^{c}\right)(1 \leq j, k \leq 3)$ hold, the pair (V, $\left.\Pi\right)$ satisfies (1.18) with $\lambda=0$, and $\|V\|_{2 r /(2-r)}+\|\nabla V\|_{4 r /(4-r)}+\left\|D^{2} V\right\|_{r}+\|\nabla \Pi\|_{r} \leq \mathfrak{C}(r)\|G\|_{r}$.

$\langle$ theoremT4.90

Theorem 4.8 Put $\mathfrak{W}_{2}:=\left\{W \in \mathcal{D}_{0}^{1,2}\left(\bar{\Omega}^{c}\right)^{3}: \operatorname{div} W=0, \partial_{1} W \in \mathcal{D}_{0}^{-1,2}\left(\bar{\Omega}^{c}\right)^{3}\right\}$. Then the operator $\mathfrak{R}: \mathfrak{W}_{2} \times L^{2}\left(\bar{\Omega}^{c}\right) \mapsto \mathcal{D}_{0}^{-1,2}\left(\bar{\Omega}^{c}\right)^{3}, \mathfrak{R}(V, \Pi)(\psi):=\int_{\bar{\Omega}^{c}}\left(\nabla V \cdot \nabla \psi+\tau \partial_{1} V \cdot \psi-\right.$ $\Pi$ div $\psi) d x$ for $\psi \in C_{0}^{\infty}\left(\bar{\Omega}^{c}\right)^{3}, V \in \mathfrak{W}_{2}, \Pi \in L^{2}\left(\bar{\Omega}^{c}\right)$, is well defined, linear and bijective, and $\|\nabla V\|_{2}+\|V\|_{4}+\left\|\partial_{1} V\right\|_{-1,2}+\|\Pi\|_{2} \leq \mathfrak{C}\|\mathfrak{R}(V, \Pi)\|_{-1,2}$ for $V \in \mathfrak{W}_{2}, \Pi \in L^{2}\left(\bar{\Omega}^{c}\right)$.

Proof: The operator $\mathfrak{R}$ is well defined due to the definition of $\mathfrak{W}_{2}$. Let $G \in \mathcal{D}_{0}^{-1,2}\left(\bar{\Omega}^{c}\right)^{3}$. By [23, Theorem VII.7.2], there is $V \in \mathcal{D}_{0}^{1,2}\left(\bar{\Omega}^{c}\right)^{3}, \Pi \in L^{2}\left(\bar{\Omega}^{c}\right)$ such that $\operatorname{div} V=0$ and (4.2) holds with $\lambda=0$ and with $\int_{\bar{\Omega}^{c}}-G \cdot \psi d x$ replaced by $-G(\psi)$. Since $\partial_{j} V_{k}, \Pi \in L^{2}\left(\bar{\Omega}^{c}\right)$ for $1 \leq j, k \leq 3$, this implies $\partial_{1} V \in \mathcal{D}_{0}^{-1,2}\left(\bar{\Omega}^{c}\right)^{3}$, so it follows that $V \in \mathfrak{W}_{2}, \mathfrak{R}(V, \Pi)=G$. The inequality stated at the end of Theorem 4.8 holds by [23, Theorem VII.7.2], and concerning the term $\left\|\partial_{1} V\right\|_{-1,2}$ - because $\mathfrak{R}(V, \Pi)=G$.

$\left\langle\right.$ theoremT4.80〉 Theorem 4.9 Let $p \in(1, \infty), \lambda \in \mathbb{C} \backslash\{0\}$ with $\Re \lambda \geq 0, G \in L^{p}\left(\bar{\Omega}^{c}\right)^{3}$. Then there is a unique function $V \in W^{2, p}\left(\bar{\Omega}^{c}\right)^{3} \cap W_{0}^{1, p}\left(\bar{\Omega}^{c}\right)^{3}$ and a function $\Pi \in W_{l o c}^{1, p}\left(\bar{\Omega}^{c}\right)$, unique up to a constant, such that $\nabla \Pi \in L^{p}\left(\bar{\Omega}^{c}\right)^{3}$, the pair $(V, \Pi)$ fulfills (1.18), and $\|V\|_{2, p}+\|\nabla \Pi\|_{p} \leq$ $\mathfrak{C}(p, \lambda)\|G\|_{p}$. 
Proof: See [32, Theorem 4.4] and the proof of this theorem on [32, p. 29].

In the rest of this section, we are going to show that if problem (2.5) only admits the trivial solution, then problem (1.19) with $\lambda=0$ (perturbed Oseen system with Dirichlet boundary conditions) possesses a $L^{2}$-weak solution for any $G \in \mathcal{D}_{0}^{-1,2}\left(\bar{\Omega}^{c}\right)^{3}$. Recall that the operator $\mathcal{B}$ was introduced in (1.12).

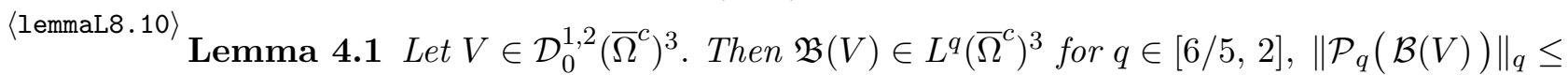
$\mathfrak{C}(q)\|\mathcal{B}(V)\|_{q} \leq \mathfrak{C}(q)\|\nabla V\|_{2}$, and

$$
\left\|\chi_{B_{R}^{c}} \mathcal{B}(V)\right\|_{q} \leq \mathfrak{C}(q)\left(\left\|U\left|B_{R}^{c}\left\|_{(1 / q-1 / 2)^{-1}}+\right\| \nabla U\right| B_{R}^{c}\right\|_{(1 / q-1 / 6)^{-1}}\right)\|\nabla V\|_{2}
$$

for $q \in[6 / 5,2], R \in(0, \infty)$ with $\bar{\Omega} \subset B_{R}$. Note that $(1 / q-1 / 2)^{-1} \in[3, \infty]$ and $(1 / q-$ $1 / 6)^{-1} \in[3 / 2,3]$ for $q$ as before, so the right-hand side of the preceding estimate is finite; see (1.5). Moreover $\mathcal{P}_{2}(\mathcal{B}(V)) \in \mathcal{D}_{0}^{-1,2}\left(\bar{\Omega}^{c}\right)^{3} \cap H_{2}\left(\bar{\Omega}^{c}\right)$.

Proof: Hölder's inequality, the estimate in Theorem 3.5 and Theorem 2.1 yield the lemma. Note that the relation $\mathcal{B}(V) \in L^{q}\left(\bar{\Omega}^{c}\right)^{3}$ for $q \in[6 / 5,2]$ implies $\mathcal{P}_{q}(\mathcal{B}(V))=\mathcal{P}_{2}(\mathcal{B}(V))$ for such $q$ (Corollary 3.7), and that $L^{6 / 5}\left(\bar{\Omega}^{c}\right)^{3} \subset \mathcal{D}_{0}^{-1,2}\left(\bar{\Omega}^{c}\right)^{3}$ (Theorem 3.6). For additional details we refer to [12, proof of Lemma 8].

Now we exploit Lemma 4.1 and Theorem 4.8 in order to construct $L^{2}$-weak solutions to (1.19) with $\lambda=0$.

$\left\langle\right.$ theoremT4.100〉 Theorem 4.10 Define $\widetilde{\Re}: \mathfrak{W}_{2} \times L^{2}\left(\bar{\Omega}^{c}\right) \mapsto \mathcal{D}_{0}^{-1,2}\left(\bar{\Omega}^{c}\right)^{3}$ by $\widetilde{\mathfrak{R}}(V, \Pi)(\psi):=\int_{\bar{\Omega}^{c}}(\nabla V \cdot \nabla \psi+$ $\left.\tau \partial_{1} V \cdot \psi-\mathcal{B}(V) \cdot \psi-\Pi \operatorname{div} \psi\right) d x$ for $\psi \in C_{0}^{\infty}\left(\bar{\Omega}^{c}\right)^{3}, V \in \mathfrak{W}_{2}, \Pi \in L^{2}\left(\bar{\Omega}^{c}\right)$, with $\mathfrak{W}_{2}$ defined in Theorem 4.8. Then $\widetilde{\mathfrak{R}}$ is well defined, linear, bounded and Fredholm with index zero.

Proof: By Lemma 4.1, we have $\mathcal{B}(V) \in \mathcal{D}_{0}^{-1,2}\left(\bar{\Omega}^{c}\right)^{3}$ for $V \in \mathcal{D}_{0}^{1,2}\left(\bar{\Omega}^{c}\right)^{3}$, so $\widetilde{\Re}$ is well defined. Due to Theorem 4.8, there is $C_{0}>0$ such that $\|\nabla V\|_{2}+\left\|\partial_{1} V\right\|_{-1,2}+\|\Pi\|_{2} \leq$ $C_{0}\|\mathfrak{R}(V, \Pi)\|_{-1,2}$ for $V, \Pi$ as in the definition of $\widetilde{\mathfrak{R}}$. For $R \in(0, \infty)$, define $\mathfrak{H}_{R}: \mathfrak{W}_{2} \times$ $L^{2}\left(\bar{\Omega}^{c}\right) \mapsto \mathcal{D}_{0}^{-1,2}\left(\bar{\Omega}^{c}\right)^{3}$ by $\mathfrak{H}_{R}(V, \Pi)(\psi):=\int_{\bar{\Omega}^{c}}-\chi_{B_{R}^{c}} \mathcal{B}(V) \cdot \psi d x$ for $\psi, V, \Pi$ as in the definition of $\widetilde{\mathfrak{R}}$. Due to (1.5), we have $\left\|\nabla U\left|B_{R}^{c}\left\|_{3 / 2}+\right\| U\right| B_{R}^{c}\right\|_{3} \rightarrow 0$ for $R \rightarrow \infty$. Since $\left\|\mathfrak{H}_{R}(V, \Pi)\right\|_{-1,2} \leq \mathfrak{C}\left\|\chi_{B_{R}^{c}} \mathcal{B}(V)\right\|_{6 / 5}$ (Theorem 3.6), we may thus conclude from Lemma 4.1 with $q=6 / 5$ that we may choose $R_{0} \in(0, \infty)$ so large that $\bar{\Omega} \subset B_{R_{0}}$ and $\left\|\mathfrak{H}_{R_{0}}(V, \Pi)\right\|_{-1,2} \leq\left(2 C_{0}\right)^{-1}\|\nabla V\|_{2}$ for $V$, $\Pi$ as in the definition of $\widetilde{\mathfrak{R}}$. Therefore from our preceding estimate of $\|\nabla V\|_{2}+\left\|\partial_{1} V\right\|_{-1,2}+\|\Pi\|_{2}$, we get $\|\nabla V\|_{2}+\left\|\partial_{1} V\right\|_{-1,2}+$ $\|\Pi\|_{2} \leq 2 C_{0}\left\|\left(\mathfrak{R}+\mathfrak{H}_{R_{0}}\right)(V, \Pi)\right\|_{-1,2}$ for $V$, $\Pi$ as before. In particular $\mathfrak{R}+\mathfrak{H}_{R_{0}}$ is oneto-one. Moreover the preceding estimates and a simple fixed point argument yield that $\mathfrak{R}+\mathfrak{H}_{R_{0}}$ is onto, and therefore bijective. Note in this respect that $\mathfrak{W}_{2}$ equipped with the mapping $V \mapsto\|\nabla V\|_{2}+\left\|\partial_{1} V\right\|_{-1,2}$ is a Banach space; see Theorem 3.5. Define $\widetilde{\mathfrak{H}}_{R_{0}}: \mathfrak{W}_{2} \times L^{2}\left(\bar{\Omega}^{c}\right) \mapsto \mathcal{D}_{0}^{-1,2}\left(\bar{\Omega}^{c}\right)^{3}$ by $\widetilde{\mathfrak{H}}_{R_{0}}(V, \Pi)(\psi):=\int_{\bar{\Omega}^{c}}-\chi_{B_{R_{0}}} \mathcal{B}(V) \cdot \psi d x$ for $\psi, V, \Pi$ as in the definition of $\widetilde{\mathfrak{R}}$. Obviously $\widetilde{\mathfrak{H}}_{R_{0}}$ is linear and bounded. Moreover we find with (3.1) that $\left\|\mathcal{B}(V) \mid \Omega_{R_{0}}\right\|_{2} \leq \mathfrak{C}\left(\|\nabla V\|_{2}\left\|U\left|\Omega_{R_{0}}\left\|_{2}+\right\| V\left\|_{6}\right\| \nabla U\right| \Omega_{R_{0}}\right\|_{6 / 5}\right) \leq \mathfrak{C}\left(R_{0}\right)\left(\|U\|_{3}+\right.$ $\left.\|\nabla U\|_{3}\right)\|\nabla V\|_{2} \leq \mathfrak{C}\left(R_{0}\right)\|\nabla V\|_{2}$, where we used that $\|U\|_{3}+\|\nabla U\|_{3}<\infty$; see (1.5). It follows with Theorem 3.3 that $\widetilde{\mathfrak{H}}_{R_{0}}$ is compact. Obviously $\mathfrak{R}+\widetilde{\mathfrak{H}}_{R_{0}}$ is linear and bounded, so that by the bijectivity of the latter operator, we may conclude that $\mathfrak{R}+\widetilde{\mathfrak{H}}_{R_{0}}+\mathfrak{H}_{R_{0}}$ is Fredholm with index zero. Since $\widetilde{\mathfrak{R}}=\mathfrak{R}+\widetilde{\mathfrak{H}}_{R_{0}}+\mathfrak{H}_{R_{0}}$, the theorem is proved. 
corollaryc4.70〉 Corollary 4.7 Suppose that any function $V \in \mathcal{D}_{0}^{1,2}\left(\bar{\Omega}^{c}\right)^{3}$ satisfying $\int_{\bar{\Omega}^{c}}\left[\nabla V \cdot \nabla \psi+\tau \partial_{1} V\right.$. $\left.\left.\psi-\mathcal{P}_{2}(\mathcal{B}(V)) \cdot \psi\right)\right] d x=0$ for $\psi \in C_{0}^{\infty}\left(\bar{\Omega}^{c}\right)^{3}$ with div $\psi=0$ vanishes. Then, for any $G \in \mathcal{D}_{0}^{-1,2}\left(\bar{\Omega}^{c}\right)^{3}$, there is a function $V \in \mathfrak{W}_{2}$ such that

$$
\left.\int_{\bar{\Omega}^{c}}\left[\nabla V \cdot \nabla \psi+\tau \partial_{1} V \cdot \psi-\mathcal{P}_{2}(\mathcal{B}(V)) \cdot \psi\right)\right] d x=G(\psi) \quad \text { for } \psi \text { as before. }
$$

Proof: Let $(V, \Pi) \in \mathfrak{W}_{2} \times L^{2}\left(\bar{\Omega}^{c}\right)^{3}$ with $\widetilde{\mathfrak{R}}(V, \Pi)=0$, where $\mathfrak{W}_{2}$ is defined in Theorem 4.8, and $\widetilde{\mathfrak{R}}$ in Theorem 4.10. Then, by the definition of $\widetilde{\mathfrak{R}}$, by Theorem 2.1 and Corollary 3.7, and because $\mathcal{B}(V) \in L^{2}\left(\bar{\Omega}^{c}\right)^{3}$ for $V \in \mathcal{D}_{0}^{1,2}\left(\bar{\Omega}^{c}\right)^{3}$ (Lemma 4.1), we see that $V$ satisfies the relation stated at the beginning of Corollary 4.7 , so $V=0$ by the assumptions in that corollary. It follows with Corollary 4.4 and 3.2 that $\Pi=0$. Thus we have shown that $\widetilde{\mathfrak{R}}$ is one-to-one. We may conclude with Theorem 4.10 that $\widetilde{\mathfrak{R}}$ is bijective. So there is $(V, \Pi) \in \mathfrak{W}_{2} \times L^{2}\left(\bar{\Omega}^{c}\right)$ with $\widetilde{\mathfrak{R}}(V, \Pi)=G$. This implies (4.6).

\section{Oseen resolvent estimates in the whole space $\mathbb{R}^{3}$.}

In this section, we extend some results from [12] concerning solutions to the Oseen resolvent (4.5) in the whole space $\mathbb{R}^{3}$. As in [12], our theory is based on the use of the fundamental solution $E^{(\lambda)}$ (see Section 2) of the scalar Oseen system. This approach has the inconvenient feature that the Helmholtz projection $P_{q}$ (Theorem 3.12) is involved when we represent solutions to (4.5) by means of convolutions with $E^{(\lambda)}$. However, although a fundamental solution to (4.5) is available $([32$, p. 19-20]), we were not able to estimate it in a satisfactory way. We begin by stating some basic facts about convolutions with $E^{(\lambda)}$, most of them taken from [12].

$\langle$ theoremT5.10〉

Theorem 5.1 Let $\lambda \in K_{\tau}$. Suppose that $H \in L^{p}\left(\mathbb{R}^{3}\right)$ for some $p \in(3, \infty)$ and for some $p \in(1,3 / 2)$. Let $\alpha \in \mathbb{N}_{0}^{3}$ with $|\alpha| \leq 1$. Then $\int_{\mathbb{R}^{3}}\left|\partial^{\alpha} E^{(\lambda)}(x-y)\right||H(y)| d y<\infty$ for any $x \in \mathbb{R}^{3}$. Let $q \in[1,2)$ and $p \in\left((1 / q-1 / 2)^{-1}, \infty\right]$ if $q \geq 3 / 2$, or $p \in((1 / q-$ $\left.1 / 2)^{-1},(1 / q-2 / 3)\right)$ if $q<3 / 2$, or $p=6$ if $q=6 / 5$. Then $\left\|\left|E^{(\lambda)}\right| *|G|\right\|_{p} \leq C(p, q)\|G\|_{q}$ for $G \in L^{q}\left(\mathbb{R}^{3}\right)$. In particular, the function $\left|E^{\lambda}(x-y)\right| *|G(y)|$ is integrable with respect to $y \in \mathbb{R}^{3}$, for a. e. $x \in \mathbb{R}^{3}$.

Let $q \in[1,3], p \in\left((1 / q-1 / 4)^{-1},(1 / q-1 / 3)\right)$ if $q<3 / 2$, or $p=2$ if $q=6 / 5$, and let $l \in\{1,2,3\}$. Then $\left\|\left|\partial_{l} E^{(\lambda)}\right| *|G|\right\|_{p} \leq C(p, q)\|G\|_{q}$ for $G \in L^{q}\left(\mathbb{R}^{3}\right)$. In particular, the function $\left|\partial_{l} E^{\lambda}(x-y)\right| *|G(y)|$ is integrable with respect to $y \in \mathbb{R}^{3}$, for a. e. $x \in \mathbb{R}^{3}$.

If $\lambda \neq 0, q \in[1,2], p \in[q, 2]$ and $q>1$ or $p<2$, then

$$
\left\|\left|E^{(\lambda)}\right| *|G|\right\|_{p} \leq C(p, q)|\lambda|^{2-4(1-1 / q+1 / p)}\|G\|_{q} \quad \text { for } G \in L^{q}\left(\mathbb{R}^{3}\right) .
$$

So also for such $p$ and $q$, the function $\left|E^{\lambda}(x-y)\right| *|G(y)|$ is integrable with respect to $y \in \mathbb{R}^{3}$, for a. e. $x \in \mathbb{R}^{3}$.

Let $\phi \in C_{0}^{\infty}\left(\mathbb{R}^{3}\right)$, and put $V:=E^{(\lambda)} * \phi$. Then $V \in C^{\infty}\left(\mathbb{R}^{3}\right), \partial^{\beta} V=E^{(\lambda)} * \partial^{\beta} \phi$ for $\beta \in \mathbb{N}_{0}^{3}, \partial_{l} V=\left(\partial_{l} E^{(\lambda)}\right) * \phi$ for $1 \leq l \leq 3,-\Delta V+\tau \partial_{1} V+\lambda V=\phi$, and $\left\|D^{2} V \mid B_{R}\right\|_{q} \leq$ $C(R, q)\|\phi\|_{q}$ for $q \in(1, \infty), R \in(0, \infty)$. 
Proof: We have $\left|\partial^{\alpha} E^{(\lambda)}(z)\right| \leq C(\tau)\left(\chi_{(0,1)}(|z|)|z|^{-1-|\alpha|}+\chi_{[1, \infty)}(|z|)|z|^{-1-|\alpha| / 2}\right.$ for $z \in$ $\mathbb{R}^{3} \backslash\{0\}, \alpha \in \mathbb{N}_{0}^{3}$ with $|\alpha| \leq 1$ by [12, Theorem 9]. Let $x \in \mathbb{R}^{3}$, and take $\alpha$ as before. Then, by Hölder's inequality and the preceding estimate, the function $\left|\partial^{\alpha} E^{(\lambda)}\right||H(y)|$ is integrable with respect to $y \in B_{1}(x)$ because $H \in L^{p}\left(\mathbb{R}^{3}\right)$ for some $p \in(3, \infty)$, and with respect to $y \in B_{1}(x)^{c}$ since $H \in L^{p}\left(\mathbb{R}^{3}\right)$ for some $p \in(1,3 / 2)$. The inequalities stated in the theorem, except the last one, hold according to [12, Theorem 10]. As for the last part of the theorem, pertaining to the function $V$, we refer to [12, Theorem 11].

$\left\langle\right.$ theoremT5.20〉 Theorem 5.2 Let $\lambda \in K_{\tau}$ and $q \in(1, \infty)$. Then $\left\|D^{2}\left(E^{(\lambda)} * \phi\right)\right\|_{q} \leq C(q)\|\phi\|_{q}$ for $\phi \in$ $C_{0}^{\infty}\left(\mathbb{R}^{3}\right)$ if $\lambda=0$ or if $q=2$. This means in particular that in the case $q=2$, the constant $C(q)$ does not depend on $\lambda$. If $\lambda \neq 0$, we further have $\left\|E^{(\lambda)} * \phi\right\|_{1, q} \leq C(q, \lambda)\|\phi\|_{q}$ for $\phi \in C_{0}^{\infty}\left(\mathbb{R}^{3}\right)$.

Proof: For $f \in L^{1}\left(\mathbb{R}^{3}\right)$, define $\hat{f}(\xi):=(2 \pi)^{-3 / 2} \int_{\mathbb{R}^{3}} e^{-i \xi \cdot \eta} f(\eta) d \eta$ for $\xi \in \mathbb{R}^{3}$ (Fourier transform of $f)$. Let $\phi \in C_{0}^{\infty}\left(\mathbb{R}^{3}\right), l, m \in\{1,2,3\}$. Then $\partial_{l} \partial_{m}\left(E^{(\varrho)} * \phi\right)=E^{(\varrho)} *\left(\partial_{l} \partial_{m} \phi\right)$ for $\varrho \in \mathbb{C}$ with $\Re \varrho \geq 0,|\varrho| \leq(\tau / 2)^{2}$ by Theorem 5.1, so we get from [14, proof of Corollary 4.1], [13, Theorem 2.1] that $\left(E^{(\varrho)} * \phi\right)(x)=\int_{\mathbb{R}^{3}} e^{-i \xi \cdot \eta}(2 \pi)^{-3 / 2}\left(\varrho+|\eta|^{2}+\right.$ $\left.i \tau \eta_{1}\right)^{-1} \eta_{l} \eta_{m} \hat{\phi}(\eta) d \eta$ for $x \in \mathbb{R}^{3}, \varrho$ as before. In this situation it shown in [23, p. 383-386] (in particular see [23, (VII.4.12)]), on the basis of Lizorkin's multiplier theorem, that the first estimate in Theorem 5.2 is valid in the case $\lambda=0$. As already noted in the proof of [12, proof of Theorem 13], we have $\left|\eta_{l} \eta_{m}\left(\varrho+|\eta|^{2}+i \tau \eta_{1}\right)^{-1}\right| \leq C$ for $\eta \in \mathbb{R}^{3} \backslash\{0\}$ because $\Re \lambda \geq 0$. Thus, if $q=2$, the above equation for $\left(E^{(\varrho)} * \phi\right)(x)$ and Plancherel's theorem yield the first estimate in Theorem 5.2, both in the case $\lambda=0$ and $\lambda \neq 0$. Now suppose that $\lambda \neq 0$. According to $[12,(3.2)]$, we have $\left|\partial^{\alpha} E^{(\lambda)}(z)\right| \leq C(\tau, \lambda)\left(\chi_{(0,1)}(|z|)|z|^{-1-|\alpha|}+\right.$ $\left.\chi_{[1, \infty)}(|z|) e^{-\sigma|\lambda|^{2}|z|}\right)$ for $z \in \mathbb{R}^{3} \backslash\{0\}, \alpha \in \mathbb{N}_{0}^{3}$ with $|\alpha| \leq 1$, where $\sigma>0$ is a constant independent of $z$ and $\lambda$. It follows that $\partial^{\alpha} E^{(\lambda)} \in L^{1}\left(\mathbb{R}^{3}\right)$ for $\alpha$ as before. Thus, by Young's inequality ([40, Part I, Theorem I.2]), we get $\left\|\left(\partial^{\alpha} E^{(\lambda)}\right) * \phi\right\|_{q} \leq C(\tau, \lambda)\|\phi\|_{q}$, for $\alpha$ as before and for $\phi \in C_{0}^{\infty}\left(\mathbb{R}^{3}\right)$, so $\left\|E^{(\lambda)} * \phi\right\|_{1, q} \leq C(\tau, \lambda)\|\phi\|_{q}$.

Corollary 5.1 Let $\lambda \in K_{\tau}$ and $G \in C_{0}^{\infty}\left(\mathbb{R}^{3}\right)^{3}$. Then $P_{2}(G) \in L^{p}\left(\mathbb{R}^{3}\right)^{3}$ for $p \in(1, \infty)$, so we may define $V:=E^{(\lambda)} * P_{2}(G)$. Put $\Pi:=Q_{2}(G)$ (Theorem 3.12). Then $V \in$ $C^{\infty}\left(\mathbb{R}^{3}\right)^{3}, \Pi \in C^{\infty}\left(\mathbb{R}^{3}\right)$, and (4.5) holds. In addition $\|V\|_{p} \leq C(p, q)\|G\|_{q},\|\nabla V\|_{p} \leq$ $C(p, q)\|G\|_{q}$, with the range of $p$ and $q$ being the same as in the corresponding estimates in Theorem 5.1, $\left\|D^{2} V \mid B_{R}\right\|_{q} \leq C(q, R)\|G\|_{q}$, for $q \in(1, \infty), R \in(0, \infty),\left\|D^{2} V\right\|_{2} \leq C\|G\|_{2}$. If $\lambda=0$, we further have $\left\|D^{2} V\right\|_{q} \leq C(q)\|G\|_{q}$ for $q \in(1, \infty)$. If $\lambda \neq 0$, the inequalities $\|V\|_{1, q} \leq C(q, \lambda)\|G\|_{q}$ for $q \in(1, \infty)$ and $|\lambda|^{-2+4(1-1 / q+1 / p)}\|V\|_{p} \leq C(p, q)\|G\|_{q}$ for $p, q$ as in the corresponding estimate in Theorem 5.1 hold. Finally $\|\nabla \Pi\|_{q} \leq C(q)\|G\|_{q}$ for $q \in(1, \infty)$.

Proof: By Theorem 3.12 and (3.3), we have $P_{2}(G), Q_{2}(G) \in C^{\infty}\left(\mathbb{R}^{3}\right)$. It further follows from Theorem 3.12 and Corollary 3.6 that $P_{2}(G) \in H_{q}\left(\mathbb{R}^{3}\right)$ and $\left\|\nabla Q_{2}(G)\right\|_{q} \leq C(q)\|G\|_{q}$ for $q \in(1, \infty)$. In particular the last inequality in Corollary 5.1 is valid. Let $p \in(1, \infty)$. By Lemma 3.1, we may choose a sequence $\left(\phi_{n}\right)$ in $C_{0}^{\infty}\left(\mathbb{R}^{3}\right)^{3}$ with $\left\|P_{2}(G)-\phi_{n}\right\|_{6 / 5} \rightarrow 0$ and $\left\|P_{2}(G)-\phi_{n}\right\|_{p} \rightarrow 0$. By Theorem 5.1 with $q=6 / 5$, we have $\left\|V-\phi_{n}\right\|_{6} \rightarrow 0$. The same reference yields $\left\|\left(\partial_{l} E^{(\lambda)}\right) * P_{2}(G)-\partial_{l}\left(E^{(\lambda)} * \phi_{n}\right)\right\|_{2} \rightarrow 0$ for $1 \leq l \leq 3$. Again by Theorem 5.1, we see that the sequence $\left(D^{2}\left(E^{(\lambda)} * \phi_{n}\right) \mid B_{R}\right)_{n>0}$ converges in $L^{p}\left(B_{R}\right)^{27}$, for any $R \in(0, \infty)$. These relations imply that $V \in W_{l o c}^{2,1}\left(\mathbb{R}^{3}\right)^{3}, \partial_{l} V=\left(\partial_{l} E^{(\lambda)}\right) * P_{2}(G)$ for 
$1 \leq l \leq 3$, and $\left\|D^{2} V-D^{2}\left(E^{(\lambda)} * \phi_{n}\right) \mid B_{R}\right\|_{p} \rightarrow 0(n \rightarrow \infty)$ for any $R \in(0, \infty)$. Now we may conclude from Theorem 5.1 that $-\Delta V+\tau \partial_{1} V+\lambda V=P_{2}(G)$. The first equation in (4.5) now follows by Theorem 3.12 and the definition of $\Pi$. Since $P_{2}(G) \in H_{6 / 5}\left(\mathbb{R}^{3}\right)$ (Corollary 3.6), we may choose a sequence $\left(\psi_{n}\right)$ in $C_{0}^{\infty}\left(\mathbb{R}^{3}\right)^{3}$ with $\operatorname{div} \psi_{n}=0$ for $n \in \mathbb{N}$ and $\left\|P_{2}(G)-\psi_{n}\right\|_{6 / 5} \rightarrow 0$. But $\partial_{l} V=\left(\partial_{l} E^{(\lambda)}\right) * P_{2}(G)$ (see above), so we may deduce from Theorem 5.1 with $q=6 / 5$ that $\left\|\partial_{l} V-\left(\partial_{l} E^{(\lambda)}\right) * \psi_{n}\right\|_{2} \rightarrow 0$ for $1 \leq l \leq 3$. We again refer to Theorem 5.1 to obtain $\operatorname{div}\left(E^{(\lambda)} * \psi_{n}\right)=E^{(\lambda)} * \operatorname{div} \psi_{n}=0$ for $n \in \mathbb{N}$. Thus we get $\operatorname{div} V=0$, so (4.5) is proved. Corollary 4.4 now yields $V \in C^{\infty}\left(\mathbb{R}^{3}\right)$. Now the estimates of $\|V\|_{p}$ and $\|\nabla V\|_{p}$ claimed in the corollary follow from Theorem 5.1, 3.12 and Corollary 3.6. As concerns the estimates of $\left\|D^{2} V \mid B_{R}\right\|_{q},\left\|D^{2} V\right\|_{2}$ and $\left\|D^{2} V\right\|_{q}$, we refer to Theorem 5.1, 5.2, 3.12 and Corollary 3.6. Finally, if $\lambda \neq 0$, the estimate of $\|V\|_{1, q}$ follows from Theorem 5.2, 3.12 and Corollary 3.6.

Corollary 5.2 Let $\lambda \in K_{\tau}$ and $q \in(1, \infty)$. Suppose that $\lambda=0$ or $q=2$. Let $p \in$ $(1,2), \quad \phi \in W_{\text {loc }}^{1,1}\left(\mathbb{R}^{3}\right) \cap L^{q}\left(\mathbb{R}^{3}\right)$ with $\nabla \phi \in L^{p}\left(\mathbb{R}^{3}\right)^{3}, l \in\{1,2,3\}$. Then $E^{(\lambda)} * \partial_{l} \phi \in$ $W^{1,1}\left(\mathbb{R}^{3}\right)$ and $\left\|\partial_{m}\left(E^{(\lambda)} * \partial_{l} \phi\right)\right\|_{q} \leq C(q)\|\phi\|_{q}(1 \leq m \leq 3)$. If $q<3$, we additionally have $E^{(\lambda)} * \partial_{l} \phi=\left(\partial_{l} E^{(\lambda)}\right) * \phi$.

Proof: By Lemma 3.1, we may choose a sequence $\left(\phi_{n}\right)$ in $C_{0}^{\infty}\left(\mathbb{R}^{3}\right)$ such that $\left\|\phi_{n}-\phi\right\|_{q} \rightarrow 0$ and $\left\|\nabla\left(\phi_{n}-\phi\right)\right\|_{p} \rightarrow 0$. Fix some $r \in\left((1 / p-1 / 2)^{-1}, \infty\right)$ with $r<(1 / p-2 / 3)^{-1}$ in the case $p<3 / 2$. Then $\left\|E^{(\lambda)} * \partial_{l} \phi-E^{(\lambda)} * \partial_{l} \phi_{n}\right\|_{r} \rightarrow 0$ by Theorem 5.1, and $\| \partial_{m} \partial_{l}\left(E^{(\lambda)} *\right.$ $\left.\phi_{n}-E^{(\lambda)} * \phi_{k}\right)\left\|_{q} \leq C(q)\right\| \phi_{n}-\phi_{k} \|_{q}$ for $n, k \in \mathbb{N}, 1 \leq m \leq 3$ by Theorem 5.2. The latter inequality implies that the sequence $\left(\partial_{m} \partial_{l}\left(E^{(\lambda)} * \phi_{n}\right)\right)_{n \geq 0}$ converges in $L^{q}\left(\mathbb{R}^{3}\right)$, for $1 \leq m \leq 3$. Moreover $\partial_{m} \partial_{l}\left(E^{(\lambda)} * \phi_{n}\right)=\partial_{m}\left(E^{(\lambda)} * \partial_{l} \phi_{n}\right)$ for $n \in \mathbb{N}, 1 \leq m \leq 3$, again by Theorem 5.1. Thus we may conclude that $E^{(\lambda)} * \partial_{l} \phi \in W_{l o c}^{1,1}\left(\mathbb{R}^{3}\right), \partial_{m}\left(E^{(\lambda)} * \partial_{l} \phi_{n}\right) \in L^{q}\left(\mathbb{R}^{3}\right)$ and $\left\|\partial_{m} \partial_{l}\left(E^{(\lambda)} * \phi_{n}\right)-\partial_{m}\left(E^{(\lambda)} * \partial_{l} \phi\right)\right\|_{q} \rightarrow 0$. Since $\left\|\partial_{m} \partial_{l}\left(E^{(\lambda)} * \phi_{n}\right)\right\|_{q} \leq C(q)\left\|\phi_{n}\right\|_{q}(n \in$ $\mathbb{N}, 1 \leq m \leq 3$ ) by Theorem 5.2, we obtain the estimate stated in Corollary 5.2. Now suppose that $q<3$. Choose $s \in\left((1 / q-1 / 4)^{-1},(1 / q-1 / 3)^{-1}\right)$. By Theorem 5.1, we get $\left\|\left(\partial_{l} E^{(\lambda)}\right) * \phi-\left(\partial_{l} E^{(\lambda)}\right) * \phi_{n}\right\|_{s} \rightarrow 0$. On the other hand, $\left(\partial_{l} E^{(\lambda)}\right) * \phi_{n}=E^{(\lambda)} * \partial_{l} \phi_{n}$ for $n \in \mathbb{N}$ by Theorem 5.1. Since $E^{(\lambda)} * \partial_{l} \phi_{n} \rightarrow E^{(\lambda)} * \partial_{l} \phi$ in $L^{r}\left(\mathbb{R}^{3}\right)^{3}$, as noted above, we thus obtain the equation stated at the end of Corollary 5.2.

For the proof of the ensuing theorem, we adapt an approach from [23, p. 391-393].

$\langle$ theoremT5.30〉

Theorem 5.3 Let $\lambda \in K_{\tau}$ and $q \in(1, \infty)$, with $\lambda=0$ or $q=2$. Let $G \in C_{0}^{\infty}\left(\mathbb{R}^{3}\right)^{3} \cap$ $\widetilde{\mathcal{D}}_{0}^{-1, q}\left(\mathbb{R}^{3}\right)^{3}$. (According to Corollary 3.1, in the case $q>3 / 2$, it suffices to require $G \in$ $\left.C_{0}^{\infty}\left(\mathbb{R}^{3}\right)^{3}\right)$. Then $\left\|\partial_{k}\left(E^{(\lambda)} * P_{2}(G)\right)\right\|_{q} \leq C(q, \tau)\|G\|_{-1, q}$. If $q<3$ and $s \in((1 / q-$ $\left.1 / 4)^{-1},(1 / q-1 / 3)^{-1}\right)$, we further have $\left\|E^{(\lambda)} * P_{2}(G)\right\|_{s} \leq C(q, s, \tau)\|G\|_{-1, q}$.

Proof: Corollary 3.6 yields $P_{2}(G) \in L^{p}\left(\mathbb{R}^{3}\right)^{3}$ for any $p \in(1, \infty)$. By referring to (3.3) and to Corollary 3.3, we get for $\psi \in C_{0}^{\infty}\left(\mathbb{R}^{3}\right)^{3}, 1 \leq l \leq 3$ that

$$
\begin{aligned}
& \left|\int_{\mathbb{R}^{3}}\left(\left(\partial_{l} N\right) * \operatorname{div} G\right) \psi d x\right|=\left|\int_{\mathbb{R}^{3}}\left(\left(\partial_{l} N\right) * G\right) \cdot \nabla \psi d x\right| \leq\left\|\left(\partial_{l} N\right) * G\right\|_{q}\|\nabla \psi\|_{q^{\prime}} \\
& \leq C(q)\|G\|_{-1, q}\|\nabla \psi\|_{q^{\prime}} .
\end{aligned}
$$

Hence $\left\|\left(\partial_{l} N\right) * \operatorname{div} G\right\|_{-1, q} \leq C(q)\|G\|_{-1, q}$ for $1 \leq l \leq 3$. Thus we may conclude from Theorem 3.12 that $\left\|P_{2}(G)\right\|_{-1, q} \leq C(q)\|G\|_{-1, q}$. Again referring to Corollary 3.3, we then 
arrive at the estimate

$$
\left\|\left(\partial_{l} N\right) * P_{2}(G)\right\|_{q} \leq C(q, \tau)\|G\|_{-1, q}(1 \leq l \leq 3) .
$$

By the first sentence of this proof and by Theorem 3.9, we know that $N * P_{2}(G) \in$ $W_{l o c}^{2,1}\left(\mathbb{R}^{3}\right)^{3},\left(\partial_{l} N\right) * P_{2}(G)=\partial_{l}\left(N * P_{2}(G)\right), D^{2}\left(N * P_{2}(G)\right) \in L^{p}\left(\mathbb{R}^{3}\right)^{27}$ for $p>1$, and

$$
\operatorname{div}\left(\left(\partial_{l} N\right) * P_{2}(G)\right)_{1 \leq l \leq 3}=P_{2}(G)
$$

In particular with the estimate of $\left\|\left(\partial_{l} N\right) * P_{2}(G)\right\|_{q}$ shown further above, we may conclude that

$$
\left(\partial_{l} N\right) * P_{2}(G) \in W_{l o c}^{1,1}\left(\mathbb{R}^{3}\right)^{3} \cap L^{q}\left(\mathbb{R}^{3}\right)^{3}, \nabla\left(\left(\partial_{l} N\right) * P_{2}(G)\right) \in L^{p}\left(\mathbb{R}^{3}\right)^{9}
$$

for any $p \in(1, \infty)$. Thus we may apply Corollary 5.2 with $\phi$ replaced by $\left(\partial_{l} N\right) * P_{2}(G)$. By (5.3), (5.2) and this corollary, we get

$$
\begin{aligned}
& \left\|\partial_{m}\left(E^{(\lambda)} * P_{2}(G)\right)\right\|_{q} \leq \sum_{l=1}^{3}\left\|\partial_{m}\left[E^{(\lambda)} * \partial_{l}\left(\left(\partial_{l} N\right) * P_{2}(G)\right)\right]\right\|_{q} \\
& \leq C(q) \sum_{l=1}^{3}\left\|\left(\partial_{l} N\right) * P_{2}(G)\right\|_{q} \leq C(q)\|G\|_{-1, q} .
\end{aligned}
$$

Thus the first estimate in Theorem 5.3 is proved. Now suppose that $q<3$ and $s \in((1 / q-$ $\left.1 / 4)^{-1},(1 / q-1 / 3)^{-1}\right)$. Then Corollary 5.2 and (5.4) yield $E^{(\lambda)} * \partial_{l}\left(\left(\partial_{l} N\right) * P_{2}(G)\right)=$ $\left(\partial_{l} E^{(\lambda)}\right) *\left(\left(\partial_{l} N\right) * P_{2}(G)\right)$, so the last inequality in Theorem 5.3 follows from Theorem 5.1 and the estimate $\left\|\left(\partial_{l} N\right) * P_{2}(G)\right\|_{q} \leq C(q, \tau)\|G\|_{-1, q}$ shown above.

Lemma 5.1 Let $q \in(1, \infty), G \in C_{0}^{\infty}\left(\mathbb{R}^{3}\right)^{3} \cap \widetilde{\mathcal{D}}_{0}^{-1, q}\left(\mathbb{R}^{3}\right)^{3}$. Then $\left\|Q_{2}(G)\right\|_{q} \leq C(q)\|G\|_{-1, q}$.

Proof: Combine Theorem 3.12, the equation $N * \operatorname{div} \phi=\sum_{l=1}^{3}\left(\partial_{l} N\right) * G$ (see (3.3)) and Corollary 3.3.

The results of this section imply the following existence results for solutions to the Oseen system and to the Oseen resolvent system (4.5), and to the system adjoint to (4.5).

Corollary 5.3 Let $\lambda \in K_{\tau}$ and $G \in C_{0}^{\infty}\left(\mathbb{R}^{3}\right)^{3}$. Put $V:=E^{(\lambda)} * P_{2}(G), \Pi:=Q_{2}(G)$. Then $V \in C^{\infty}\left(\mathbb{R}^{3}\right)^{3}, \Pi \in C^{\infty}\left(\mathbb{R}^{3}\right)$, and the pair $(V, \Pi)$ solves (4.5). Moreover, if $\lambda \neq 0$, we have $V \in W^{1, p}\left(\mathbb{R}^{3}\right)$ for any $p \in(1, \infty)$, and $D^{2} V \in L^{2}\left(\mathbb{R}^{3}\right)^{27}$. If $\lambda=0$, we have $V \in L^{p}\left(\mathbb{R}^{3}\right)^{3}$ for $p \in(2, \infty), \nabla V \in L^{p}\left(\mathbb{R}^{3}\right)^{9}$ for $p \in(4 / 3, \infty)$, and $D^{2} V \in L^{p}\left(\mathbb{R}^{3}\right)^{27}$ for $p \in(1, \infty)$.

If $\lambda=0$ and $G \in C_{0}^{\infty}\left(\mathbb{R}^{3}\right)^{3} \cap \widetilde{\mathcal{D}}_{0}^{-1, p}\left(\mathbb{R}^{3}\right)^{3}$ for any $p \in(1, \infty)$, we get $V \in L^{p}\left(\mathbb{R}^{3}\right)^{3}$ for $p \in(4 / 3, \infty)$ and $\nabla V \in L^{p}\left(\mathbb{R}^{3}\right)^{9}$ for $p \in(1, \infty)$. In all cases we have $\Pi \in L^{p}\left(\mathbb{R}^{3}\right)$ for $p \in(3 / 2, \infty)$ and $\nabla \Pi \in L^{p}\left(\mathbb{R}^{3}\right)^{3}$ for $p \in(1, \infty)$. If $G \in C_{0}^{\infty}\left(\mathbb{R}^{3}\right)^{3} \cap \widetilde{\mathcal{D}}_{0}^{-1, p}\left(\mathbb{R}^{3}\right)^{3}$ for any $p \in(1, \infty)$, we get $\Pi \in L^{p}\left(\mathbb{R}^{3}\right)$ for any $p \in(1, \infty)$.

Put $\widetilde{V}(x):=V(-x), \widetilde{\Pi}(x):=\Pi(-x)$ for $x \in \mathbb{R}^{3}$. Then all the preceding statements remain valid with $\widetilde{V}, \widetilde{\Pi}$ in the role of $V$ and $\Pi$, respectively, except that the factor $\tau$ in (4.5) has to be replaced by $-\tau$. 
Proof: According to Corollary 5.1, we have $V_{j}, \Pi \in C^{\infty}\left(\mathbb{R}^{3}\right)$ for $1 \leq j \leq 3$, and the pair $(V, \Pi)$ solves $(4.5)$. If $\lambda \neq 0$, Corollary 5.1 yields that $V \in W^{1, p}\left(\mathbb{R}^{3}\right)^{3}$ for $p \in(1, \infty)$ and $D^{2} V \in L^{2}\left(\mathbb{R}^{3}\right)^{27}$. In the case $\lambda=0$, the integrability relations stated for $V, \nabla V$ and $D^{2} V$ follow from Corollary 5.1, and if $G \in C_{0}^{\infty}\left(\mathbb{R}^{3}\right)^{3} \cap \widetilde{\mathcal{D}}_{0}^{-1, p}\left(\mathbb{R}^{3}\right)^{3}$ for $p \in(1, \infty)$, from Theorem 5.3. Due to Theorem 3.12, (3.3) and the second last statement of Theorem 3.9, we know that $\Pi \in L^{p}\left(\mathbb{R}^{3}\right)$ for $p \in(3 / 2, \infty)$. In the case $G \in C_{0}^{\infty}\left(\mathbb{R}^{3}\right)^{3} \cap \widetilde{\mathcal{D}}_{0}^{-1, p}\left(\mathbb{R}^{3}\right)^{3}$ for $p \in(1, \infty)$, Lemma 5.1 yields $\Pi \in L^{p}\left(\mathbb{R}^{3}\right)$ for this range of $p$. $L^{p}$-integrability of $\nabla \Pi$ for any $p \in(1, \infty)$ holds according to Corollary 5.1.

\section{Uniqueness theorems for solutions to (1.18).}

We first consider the whole space case, then exploit the results we obtain for this case to determine a uniqueness class for weak solutions of (1.18) (exterior domain case). Our results are more general than what is available in literature because we do not suppose that the gradient of the velocity is an $L^{p}$-function for a single $p \in(1, \infty)$. Instead it may

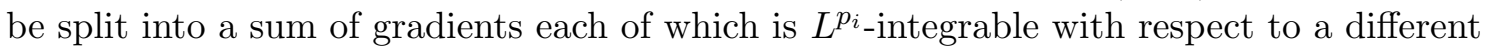
exponent $p_{i} \in(1, \infty)$. Unfortunately this seemingly small generalization complicates the argument considerably. For technical reasons, we first consider a splitting into a sum of three terms (whole space case), and then into a sum of two terms (exterior domain case).

Theorem 6.1 Let $\lambda \in K_{\tau}$. For $i \in\{1,2,3\}$, let $q_{i}, r_{i} \in(1, \infty), R_{i} \in(0, \infty)$ and $V^{(i)} \in$ $W_{\text {loc }}^{1,1}\left(\mathbb{R}^{3}\right)^{3}$ with $V^{(i)} \mid B_{R_{i}}^{c} \in L^{r_{i}}\left(B_{R_{i}}^{c}\right)^{3}, \nabla V^{(i)} \in L^{q_{i}}\left(\mathbb{R}^{3}\right)^{9}$. Suppose that div $\left(\sum_{i=1}^{3} V^{(i)}\right)=0$ and (4.1) is satisfied with $A=\mathbb{R}^{3}, G=0, V=\sum_{i=1}^{3} V^{(i)}$. Then $\sum_{i=1}^{3} V^{(i)}=0$.

Proof: Abbreviate $V:=\sum_{i=1}^{3} V^{(i)}, q:=\min \left\{q_{1}, q_{2}, q_{3}\right\}$. Then $V \in W_{\text {loc }}^{1,1}\left(\mathbb{R}^{3}\right)^{3}, \nabla V \in$ $L_{l o c}^{\widetilde{q}}\left(\mathbb{R}^{3}\right)^{9}, \operatorname{div} V=0$, and (4.1) is satisfied with $A=\mathbb{R}^{3}, G=0$. Corollary 4.4 yields that $V \in C^{\infty}\left(\mathbb{R}^{3}\right)^{3}$, and that there is $\Pi \in C^{\infty}\left(\mathbb{R}^{3}\right)$ such that (4.5) is valid with $G=0$.

Fix some function $\varphi \in C_{0}^{\infty}\left(B_{7 / 4}\right)^{3}$ with $\varphi \mid B_{5 / 4}=1,0 \leq \varphi \leq 1$, and put $\varphi_{n}(x):=$ $\varphi((1 / n) x)$ for $n \in \mathbb{N}, x \in \mathbb{R}^{3}$. Note that $\varphi_{n} \in C_{0}^{\infty}\left(B_{7 n / 4}\right), 0 \leq \varphi_{n} \leq 1, \varphi \mid B_{5 n / 4}=1$,

$$
|\nabla \varphi|_{\infty} \leq \mathfrak{C} n^{-1}, \quad\left|\partial_{l} \partial_{m} \varphi\right|_{\infty} \leq \mathfrak{C} n^{-2} \text { for } n \in \mathbb{N}, 1 \leq l, m \leq 3 .
$$

Let $l \in\{1,2,3\}, \phi \in C_{0}^{\infty}\left(\mathbb{R}^{3}\right)^{3}$. Obviously $\partial_{l} \phi \in C_{0}^{\infty}\left(\mathbb{R}^{3}\right)^{3} \cap \widetilde{\mathcal{D}}_{0}^{-1, p}\left(\mathbb{R}^{3}\right)^{3}$ for any $p \in(1, \infty)$. Therefore, according to Corollary 5.3, we may choose functions $W \in C^{\infty}\left(\mathbb{R}^{3}\right)^{3}, \Gamma \in$ $C^{\infty}\left(\mathbb{R}^{3}\right)$ such that the pair $(W, \Gamma)$ solves (4.5) with $W, \Gamma, \partial_{l} \phi,-\tau$ in the place of $V, \Pi, G, \tau$, respectively, and such that $\partial_{j} W_{k}, \Gamma \in L^{p}\left(\mathbb{R}^{3}\right)$ for $p \in(1, \infty), 1 \leq j, k \leq 3$. In the case $\lambda \neq 0$, we may require $W \in L^{p}\left(\mathbb{R}^{3}\right)^{3}$ even for any $p \in(1, \infty)$. On taking account of the fact that $\phi$ has compact support, we get

$$
\begin{aligned}
& \int_{\mathbb{R}^{3}} \partial_{l} V \cdot \phi d x=-\int_{\mathbb{R}^{3}} V \cdot \partial_{l} \phi d x=-\lim _{n \rightarrow \infty} \int_{\mathbb{R}^{3}} V \cdot \partial_{l} \phi \varphi_{n} d x \\
& =-\lim _{n \rightarrow \infty} \int_{\mathbb{R}^{3}} V \cdot\left(-\Delta W-\tau \partial_{1} W+\lambda W+\nabla \Gamma\right) \varphi_{n} d x .
\end{aligned}
$$


Let $n \in \mathbb{N}$, and abbreviate $A_{n}:=B_{2 n} \backslash \overline{B_{n}}$. Since $\operatorname{div} W=0$ and $\operatorname{supp}\left(\nabla \varphi_{n}\right) \subset A_{n}$, we have $\nabla \varphi_{n} \cdot W \in C_{0}^{\infty}\left(A_{n}\right)$ and $\int_{A_{n}} \nabla \varphi_{n} \cdot W d x=0$. Thus we may refer to Theorem 3.7 to define $\mathfrak{D}_{n}:=n \mathfrak{D}(2,1,1,2)\left[\left(\left(-\nabla \varphi_{n} \cdot W\right)(n \cdot) \mid A_{n}\right]((1 / n) x)\right.$ for $x \in \mathbb{R}^{3}$. Theorem 3.7 and Lemma 3.5 yield that $\mathfrak{D}_{n} \in C_{0}^{\infty}\left(A_{n}\right)^{3}$ and $\operatorname{div}\left(\varphi_{n} \cdot W+\mathfrak{D}_{n}\right)=0$. It further follows from Theorem 3.7, in particular its last statement, and from Lemma 3.5 and (6.1) that

$$
\left\|\mathfrak{D}_{n}\right\|_{p} \leq \mathfrak{C}(p)\left\|W\left|A_{n}\left\|_{p}, \quad\right\| \partial^{\alpha} \mathfrak{D}_{n}\left\|_{p} \leq \mathfrak{C}(p) n^{-1}\right\| W\right| A_{n}\right\|_{1, p},
$$

for any $\alpha \in \mathbb{N}_{0}^{3}$ with $1 \leq|\alpha| \leq 2$, and for any $p \in(1, \infty)$. On the other hand, since $V_{j}, \Pi \in C^{\infty}\left(\mathbb{R}^{3}\right)$ for $1 \leq j \leq 3$, equation (4.5) is valid with $G=0$. Observing that $\varphi_{n} W+\mathfrak{D}_{n} \in C_{0}^{\infty}\left(B_{2 n}\right)^{3}$, and recalling that $\operatorname{div}\left(\varphi_{n} W+\mathfrak{D}_{n}\right)=0$ for $n \in \mathbb{N}$, we may thus deduce from (6.2) by some integrations by parts that $\int_{\mathbb{R}^{3}} \partial_{l} V \cdot \phi d x=\lim _{n \rightarrow \infty} \mathfrak{A}_{n}$, with

$$
\begin{aligned}
\mathfrak{A}_{n}:= & -\int_{\mathbb{R}^{3}} V \cdot\left(2 \sum_{k=1}^{3} \partial_{k} \varphi_{n} \partial_{k} W+\Delta \varphi_{n} W+\tau \partial_{1} \varphi_{n} W+\Delta \mathfrak{D}_{n}-\tau \partial_{1} \mathfrak{D}_{n}\right. \\
& \left.-\lambda \mathfrak{D}_{n}-\Gamma \nabla \varphi_{n}\right) d x \quad \text { for } n \in \mathbb{N} .
\end{aligned}
$$

Since the support of any derivative of $\varphi_{n}$ is a subset of $A_{n}$, and because of (6.1), we get for $n \in \mathbb{N}$ with $n \geq R_{0}:=\max \left\{R_{1}, R_{2}, R_{3}\right\}$ that

$$
\begin{aligned}
\left|\mathfrak{A}_{n}\right| \leq & C \sum_{i=1}^{3}\left\|V^{(i)} \mid B_{n}^{c}\right\|_{r_{i}}\left(\left\|\nabla W\left|A_{n}\left\|_{r_{i}^{\prime}}+n^{-1}\right\| W\right| A_{n}\right\|_{r_{i}^{\prime}}+\sum_{\alpha \in \mathbb{N}_{0}^{3}, 1 \leq|\alpha| \leq 2}\left\|\partial^{\alpha} \mathfrak{D}_{n}\right\|_{r_{i}^{\prime}}\right. \\
& \left.+|\lambda|\left\|\mathfrak{D}_{n}\right\|_{r_{i}^{\prime}}+\left\|\Gamma \mid A_{n}\right\|_{r_{i}^{\prime}}\right) .
\end{aligned}
$$

Next we apply (6.3) to obtain

$$
\begin{aligned}
\left|\mathfrak{A}_{n}\right| \leq & C\left(q_{1}, q_{2}, q_{3}\right) \sum_{i=1}^{3}\left\|V^{(i)} \mid B_{n}^{c}\right\|_{r_{i}}\left(\left\|\nabla W\left|A_{n}\left\|_{r_{i}^{\prime}}+n^{-1}\right\| W\right| A_{n}\right\|_{1, r_{i}^{\prime}}\right. \\
& \left.+|\lambda|\left\|W\left|A_{n}\left\|_{r_{i}^{\prime}}+\right\| \Gamma\right| A_{n}\right\|_{r_{i}^{\prime}}\right) \quad\left(n \in \mathbb{N}, n \geq R_{0}\right) .
\end{aligned}
$$

Lemma 3.3 yields that $n^{-1}\left\|W \mid A_{n}\right\|_{r_{i}^{\prime}}$ is bounded by $C\left(r_{i}^{\prime}\right)\left(\left\|W\left|{\overline{B_{n}}}^{c}\left\|_{3}+\right\| \nabla W\right|{\overline{B_{n}}}^{c}\right\|_{r_{i}^{\prime}}+\right.$ $n^{-1}\|W\|_{3}+n^{-1}\|\nabla W\|_{q_{i}^{\prime}}$ for $n \in \mathbb{N}, n \geq R_{0}$. So we may deduce from (6.4) that

$$
\begin{aligned}
\left|\mathfrak{A}_{n}\right| \leq & C\left(q_{1}, q_{2}, q_{3}\right) \sum_{i=1}^{3}\left\|V^{(i)} \mid B_{n}^{c}\right\|_{r_{i}}\left(\| W | B _ { n } ^ { c } \| _ { 3 } + \| \nabla W | B _ { n } ^ { c } \| _ { r _ { i } ^ { \prime } } + n ^ { - 1 } \| W \| _ { 3 } \quad ( 6 . 5 ) \longdiv { \mathrm { T } 6 . 1 0 . 5 0 }\right. \\
& \left.+n^{-1}\|\nabla W\|_{r_{i}^{\prime}}+|\lambda|\left\|W\left|A_{n}\left\|_{r_{i}^{\prime}}+\right\| \Gamma\right| B_{n}^{c}\right\|_{r_{i}^{\prime}}\right) \quad\left(n \in \mathbb{N}, n \geq R_{0}\right) .
\end{aligned}
$$

As the reader may recall, we have $W \in L^{p}\left(\mathbb{R}^{3}\right)^{3}$ for $p \in(4 / 3, \infty), \quad \nabla W \in L^{p}\left(\mathbb{R}^{3}\right)^{3}$ for $p>1$. Thus we may conclude that $\left\|\nabla W\left|B_{n}^{c}\left\|_{r_{i}^{\prime}}+\right\| W\right| B_{n}^{c}\right\|_{3} \rightarrow 0$ and $n^{-1}\|W\|_{3}+$ $n^{-1}\|\nabla W\|_{r_{i}^{\prime}} \rightarrow 0(n \rightarrow \infty)$ for $1 \leq i \leq 3$. Similarly, recalling that $\Gamma \in L^{p}\left(\mathbb{R}^{3}\right)$ for $p \in(1, \infty)$, we get $\left\|\Gamma \mid B_{n}^{c}\right\|_{r_{i}^{\prime}} \rightarrow 0(n \rightarrow \infty, 1 \leq i \leq 3)$. Finally, if $\lambda \neq 0$, we have $W \in L^{p}\left(\mathbb{R}^{3}\right)^{3}$ for $p>1$, so $|\lambda|\left\|W \mid A_{n}\right\|_{r_{i}^{\prime}} \rightarrow 0(n \rightarrow \infty, 1 \leq i \leq 3)$. Therefore inequality (6.5) implies that $\left|\mathfrak{A}_{n}\right| \rightarrow 0$. Since $\int_{\mathbb{R}^{3}} \partial V \cdot \phi d x=\lim _{n \rightarrow \infty} \mathfrak{A}_{n}$, as observed above, this 
means that $\int_{\mathbb{R}^{3}} \partial_{l} V \cdot \phi d x=0$. But the function $\phi$ was arbitrarily chosen in $C_{0}^{\infty}\left(\mathbb{R}^{3}\right)^{3}$, so $\partial_{l} V=0$. This is true for any $l \in\{1,2,3\}$. We thus see there is $c \in \mathbb{C}^{3}$ with $V=c$ (Corollary 3.2). But for $\epsilon>0, i \in\{1,2,3\}$, the relation $\left|\left\{x \in B_{R_{0}}^{c}:\left|V^{(i)}(x)\right| \geq \epsilon\right\}\right|<\infty$ holds because otherwise the assumption $V \mid B_{R_{i}}^{c} \in L^{r_{i}}\left(B_{R_{i}}^{c}\right)^{3}$ could not be true. Now suppose that $c \neq 0$. Then $\left\{x \in B_{R_{0}}^{c}: V(x)=c\right\} \subset \cup_{i=1}^{3}\left\{x \in B_{R_{0}}^{c}:\left|V^{(i)}(x)\right| \geq|c| / 6\right\}$. On the other hand, the equation $V=c$ means that $\left\{x \in B_{R_{0}}^{c}: V(x)=c\right\}=B_{R_{0}}^{c}$, so there must be a number $i \in\{1,2,3\}$ with $\left|\left\{x \in B_{R_{0}}^{c}:\left|V^{(i)}(x)\right| \geq|c| / 6\right\}\right|=\infty$, a contradiction! Therefore $c=0$, so the theorem is proved.

Now we consider the exterior domain case (weak solutions to problem (1.18)).

Theorem 6.2 Let $\lambda \in K_{\tau}$ and $R_{0} \in(0, \infty)$ with $\bar{\Omega} \subset B_{R_{0}}$. For $i \in\{1,2\}$, let $q_{i}, s_{i} \in$ $(1, \infty), \quad V^{(i)} \in W_{\text {loc }}^{1,1}\left(\bar{\Omega}^{c}\right)^{3}$ with $\nabla V^{(i)} \in L^{q_{i}}\left(\bar{\Omega}^{c}\right)^{9}$, and $V^{(i)} \mid B_{R_{0}}^{c} \in L^{s_{i}}\left(B_{R_{0}}^{c}\right)^{3}$. Further suppose that $\operatorname{div}\left(V^{(1)}-V^{(2)}\right)=0, V^{(1)}-V^{(2)} \mid \partial \Omega=0$, and that (4.1) holds with $A=$ $\bar{\Omega}^{c}, G=0, V=V^{(1)}-V^{(2)}$. Then $V^{(1)}-V^{(2)}=0$.

Proof: Put $V:=V^{(1)}-V^{(2)}, q:=\min \left\{q_{1}, q_{2}\right\}$. Then $V \in W_{l o c}^{1,1}\left(\bar{\Omega}^{c}\right)^{3}, \nabla V \in L_{l o c}^{q}\left(\bar{\Omega}^{c}\right)^{9}, V$ is solenoidal, and equation (4.1) holds with $A=\bar{\Omega}^{c}, G=0$. In particular, there is a pressure $\Pi \in L_{l o c}^{q}\left(\bar{\Omega}^{c}\right)$ associated with $V$ (Theorem 4.2). From Corollary 4.4, we may conclude that $V_{j}, \Pi \in C^{\infty}\left(\bar{\Omega}^{c}\right)(1 \leq j \leq 3)$, and equation (4.5) is valid with $G=0$. In addition, Corollary 4.3 yields that

$V\left|\Omega_{R} \in W^{2, p}\left(\Omega_{R}\right)^{3}, \Pi\right| \Omega_{R} \in W^{1, p}\left(\Omega_{R}\right)^{3}$, for $R \in(0, \infty)$ with $\bar{\Omega} \subset B_{R}, p \in(1, \infty)$. (6.6) T6.20.10

Fix a function $\varphi \in C^{\infty}\left(\mathbb{R}^{3}\right)$ with $0 \leq \varphi \leq 1, \varphi\left|B_{R_{0}+1 / 4}=0, \varphi\right| B_{R_{0}+3 / 4}^{c}=1$, and abbreviate $B:=B_{R_{0}+1} \backslash \overline{B_{R_{0}}}$. By Theorem 3.1 and our assumptions on $V^{(i)}$, we get

$$
\nabla\left(\varphi V^{(i)}\right) \in L^{q_{i}}\left(\mathbb{R}^{3}\right)^{9}, \varphi V^{(i)}\left|B_{R_{0}+1}^{c}=V^{(i)}\right| B_{R_{0}+1}^{c} \in L^{s_{i}}\left(B_{R_{0}+1}^{c}\right)^{3}(i \in\{1,2\}) .
$$

Since $\nabla \varphi \cdot V \in C_{0}^{\infty}(B)$ and $\operatorname{div} V=0$, we have $\int_{B} \nabla \varphi \cdot V d x=0$. Thus we may apply Theorem 3.7, setting $\mathfrak{D}:=\mathfrak{D}\left(2,1, R_{0}, R_{0}+1\right)(-\nabla \varphi \cdot V \mid B)$. Note that $\mathfrak{D} \in C_{0}^{\infty}(B)^{3}$. We further define $\widetilde{V}, \widetilde{\Pi}, \widetilde{F}$ as the zero extension to $\mathbb{R}^{3}$ of $(1-\varphi) V+\mathfrak{D},(1-\varphi) \Pi$ and $-2 \sum_{k=1}^{3} \partial_{k} \varphi \partial_{k} V-\Delta \varphi V+\tau \partial_{1} \varphi V-\Delta \mathfrak{D}+\tau \partial_{1} \mathfrak{D}+\lambda \mathfrak{D}+\Pi \nabla \varphi$, respectively. By the choice of $\mathfrak{D}$ and Theorem 3.7, we have $\operatorname{div} \widetilde{V}=0$. Moreover $\widetilde{V}_{j}, \widetilde{\Pi} \in C^{\infty}\left(\mathbb{R}^{3}\right)(1 \leq j \leq 3), \widetilde{F} \in$ $C_{0}^{\infty}(B)^{3}$, the pair $(\widetilde{V}, \widetilde{\Pi})$ satisfies $(4.5)$ with $V, \Pi, G$ replaced by $\widetilde{V}, \widetilde{\Pi}, \widetilde{F}$, respectively. Since $\widetilde{F} \in C_{0}^{\infty}(B)^{3}$, we may apply Corollary 4.5, allowing us to choose functions $W \in$ $C^{\infty}\left(\mathbb{R}^{3}\right)^{3}, \Gamma \in C^{\infty}\left(\mathbb{R}^{3}\right)$ such that the pair $(W, \Gamma)$ solves $(4.5)$ with $V, \Pi, G$ replaced by $W, \Gamma, \widetilde{F}$, respectively, and such that in addition $W \in W^{2,2}\left(\mathbb{R}^{3}\right)^{3}$ in the case $\lambda \neq 0$, and $W \in L^{p}\left(\mathbb{R}^{3}\right)^{3}$ for $p \in(2, \infty), \nabla W \in L^{p}\left(\mathbb{R}^{3}\right)^{9}$ for $p \in(4 / 3, \infty), D^{2} W \in L^{p}\left(\mathbb{R}^{3}\right)^{27}$ for $p \in(1, \infty)$ if $\lambda=0$. At this point we may conclude that $\varphi V^{(1)}-\varphi V^{(2)}-W+\mathfrak{D}=$ $\widetilde{V}-W \in W_{l o c}^{1,1}\left(\mathbb{R}^{3}\right)^{3}, \operatorname{div}(\widetilde{V}-W)=0$, and the function $\widetilde{V}-W$ satisfies (4.1) with $A=\mathbb{R}^{3}, G=0, V=\widetilde{V}-W$. Recalling (6.7) and the relations $\mathfrak{D} \in C_{0}^{\infty}(B), W \in$ $C^{\infty}\left(\mathbb{R}^{3}\right)^{3} \cap L^{p}\left(\mathbb{R}^{3}\right)$ for $p>2$, we see we may apply Theorem 6.1 with $V^{(1)}, V^{(2)}, V^{(3)}$ replaced by $\varphi V^{(1)},-\varphi V^{(2)},-W+\mathfrak{D}$, respectively. This theorem yields that $\varphi V^{(1)}-$ $\varphi V^{(2)}-W+\mathfrak{D}=0$, that is, $\widetilde{V}-W=0$. As a consequence, in view of (6.6), we have $V\left|B_{R_{0}+1}^{c}=\widetilde{V}\right| B_{R_{0}+1}^{c}=W \mid B_{R_{0}+1}^{c}$. This equation, (6.6) and the integrability properties of $W$ listed above yield that $V \in W^{2,2}\left(\bar{\Omega}^{c}\right)^{3}$ if $\lambda \neq 0$, as well as $V \in L^{p}\left(\bar{\Omega}^{c}\right)^{3}$ for $p \in$ 
$(2, \infty), \nabla V \in L^{p}\left(\bar{\Omega}^{c}\right)^{9}$ for $p \in(4 / 3, \infty), D^{2} V \in L^{p}\left(\bar{\Omega}^{c}\right)^{27}$ for $p \in(1, \infty)$ if $\lambda=0$. Now choose $p=2$ in the case $\lambda \neq 0$, and $p=3$ if $\lambda=0$. Then, by the integrability properties of $V$ we have just proved, it follows that $V \in W^{1, p}\left(\bar{\Omega}^{c}\right)^{3}$ and $\nabla V \in L^{p^{\prime}}\left(\bar{\Omega}^{c}\right)^{9}$ Since $V \mid \partial \Omega=0$ by our assumptions, we get $V \in W_{0}^{1, p}\left(\bar{\Omega}^{c}\right)^{3}$. But $\operatorname{div} V=0$ also by our assumptions, so Theorem 3.2 yields there is a sequence $\left(\phi_{n}\right)$ in $C_{0}^{\infty}\left(\bar{\Omega}^{c}\right)^{3}$ with $\operatorname{div} \phi_{n}=0$ for $n \in \mathbb{N}$ and $\left\|V-\phi_{n}\right\|_{1, p} \rightarrow 0$. Using $\overline{\phi_{n}}$ as test function in (4.1), letting $n$ tend to infinity, and taking account of the fact that $\nabla V \in L^{p^{\prime}}(\bar{\Omega})^{9}$, we arrive at an equation whose real part is given by $\|V\|_{2}^{2}+\Re \int_{\bar{\Omega}^{c}} \partial_{1} V \cdot \bar{V} d x+\gamma(V)=0$, where $\gamma(V)=0$ if $\lambda=0$, and $\gamma(V):=\Re \lambda\|V\|_{2}^{2}$ else. Fix some function $\psi \in C_{0}^{\infty}\left(B_{2}\right)$ with $\psi \mid B_{1}=1$, and define $\psi_{n}(x):=\psi\left(n^{-1} x\right)$ for $x \in \mathbb{R}^{3}, n \in \mathbb{N}$. Then $\psi_{n} \in C_{0}^{\infty}\left(B_{2 n}\right), \psi_{n} \mid B_{n}=1$, and $\left|\nabla \psi_{n}\right|_{\infty} \leq \mathfrak{C} n^{-1}$. On the other hand, since $\partial_{1} V \in L^{p^{\prime}}\left(\bar{\Omega}^{c}\right)^{3}, V \in L^{p}\left(\bar{\Omega}^{c}\right)^{3}$ by our choice of $p$, hence $\partial_{1} V \cdot \bar{V} \in L^{1}\left(\bar{\Omega}^{c}\right)^{3}$, we have $\int_{\bar{\Omega}^{c}} \partial_{1} V \cdot \bar{V} d x=\lim _{n \rightarrow \infty} \int_{\bar{\Omega}^{c}} \partial_{1} V \cdot \bar{V} \psi_{n} d x$. But $V \in C^{\infty}\left(\mathbb{R}^{3}\right)^{3}$, so obviously

$$
\Re \int_{\bar{\Omega}^{c}} \partial_{1} V \cdot \bar{V} \psi_{n} d x=\Re \int_{\bar{\Omega}^{c}}\left[\partial_{1}(\Re V) \cdot \Re V+\partial_{1}(\Im V) \cdot \Im V\right] \psi_{n} d x=-\int_{\bar{\Omega}^{c}}|V|^{2} \partial_{1} \psi d x / 2 .
$$

It is also obvious that $|V|^{2} \in L^{r}\left(\bar{\Omega}^{c}\right)$ for any $r \in(1, \infty)$, and $\left(\int \bar{\Omega}^{c}\left|\partial_{1} \psi_{n}\right|^{s} d x\right)^{1 / s} \leq$ $\mathfrak{C} n^{-1+3 / s}$ for $s \in(1, \infty)$. Therefore $\int_{\bar{\Omega}^{c}}|V|^{2} \partial_{1} \psi d x \rightarrow 0$ for $n \rightarrow \infty$, so we obtain $\|\nabla V\|_{2}^{2}+$ $\gamma(V)=0$. As a consequence $\nabla V=0$, and we may conclude with Corollary 3.2 that $V=0$.

\section{Oseen resolvent estimates.}

In this section, we derive some estimates of solutions to (1.18), with upper bounds depending on $\lambda$ in an explicit way ("resolvent estimates"). We begin by presenting two corollaries where we collect some features of these solutions following immediately from the existence results in Theorem 4.7 and 4.9, and from the uniqueness properties stated in Theorem 6.2 and Corollary 3.2. The notation we introduce for these solutions in the two corollaries in question will be used frequently in the rest of this paper.

Corollary 7.1 Let $\lambda \in K_{\tau} \backslash\{0\}$. For any $G \in \cup_{q \in(1, \infty)} L^{q}\left(\bar{\Omega}^{c}\right)^{3}$ or $G \in \cup_{q \in(1, \infty)} L^{q}\left(\mathbb{R}^{3}\right)^{3}$, there is a unique function $V:=\mathfrak{V}(\lambda, G) \in \cup_{q \in(1, \infty)} W^{2, q}\left(\bar{\Omega}^{c}\right)^{3}$ and a unique function $\Pi:=\Pi(\lambda, G) \in \cup_{q \in(1, \infty)} W_{\text {loc }}^{1, q}\left(\bar{\Omega}^{c}\right)$ such that $\int_{\Omega_{S+1}} \Pi d x=0$ and the pair $(V, \Pi)$ solves (1.18), with $G \mid \bar{\Omega}^{c}$ instead of $G$ if $G \in \cup_{q \in(1, \infty)} L^{q}\left(\mathbb{R}^{3}\right)^{3}$. If $p \in(1, \infty)$ and $G \in L^{p}\left(\bar{\Omega}^{c}\right)^{3} \cup$ $L^{p}\left(\mathbb{R}^{3}\right)^{3}$, then $V \in W^{2, p}\left(\bar{\Omega}^{c}\right)^{3}$ and $\nabla \Pi \in L^{p}\left(\bar{\Omega}^{c}\right)^{3}$. In particular, if $G \in C_{0}^{\infty}\left(\bar{\Omega}^{c}\right)^{3}$, we have $V \in W^{2, p}\left(\bar{\Omega}^{c}\right)^{3}$ and $\nabla \Pi \in L^{p}\left(\bar{\Omega}^{c}\right)^{3}$ for any $p \in(1, \infty)$.

Corollary 7.2 For any $G \in \cup_{q \in(1,2)} L^{q}\left(\bar{\Omega}^{c}\right)^{3}$ or $G \in \cup_{q \in(1,2)} L^{q}\left(\mathbb{R}^{3}\right)^{3}$, there is a unique function $V:=\mathfrak{V}(0, G) \in W_{\text {loc }}^{2,1}\left(\bar{\Omega}^{c}\right)^{3}$ and a unique function $\Pi:=\Pi(0, G) \in W_{\text {loc }}^{1,1}\left(\bar{\Omega}^{c}\right)$ such that $\int_{\Omega_{S+1}} \Pi d x=0, \quad V \in \cup_{q \in(2, \infty)} L^{q}\left(\bar{\Omega}^{c}\right)^{3}, \quad \nabla V \in \cup_{q \in(4 / 3, \infty)} L^{q}\left(\bar{\Omega}^{c}\right)^{9}, \quad D^{2} V \in$ $\cup_{q \in(1, \infty)} L^{q}\left(\bar{\Omega}^{c}\right)^{27}, \nabla \Pi \in \cup_{q \in(1, \infty)} L^{q}\left(\bar{\Omega}^{c}\right)^{3}$, and such that (1.18) holds with $\lambda=0$, and with with $G \mid \bar{\Omega}^{c}$ instead of $G$ if $G \in \cup_{q \in(1,2)} L^{q}\left(\mathbb{R}^{3}\right)^{3}$. If $p \in(1,2)$ and $G \in L^{p}\left(\bar{\Omega}^{c}\right)^{3} \cup L^{p}\left(\mathbb{R}^{3}\right)^{3}$, then $V \in L^{2 p /(2-p)}\left(\bar{\Omega}^{c}\right)^{3}, \quad \nabla V \in L^{4 p /(4-p)}\left(\bar{\Omega}^{c}\right)^{9}, D^{2} V \in L^{p}\left(\bar{\Omega}^{c}\right)^{27}, \nabla \Pi \in L^{p}\left(\bar{\Omega}^{c}\right)^{3}$, in particular $V \mid \Omega_{R} \in W^{2, p}\left(\Omega_{R}\right)^{3}$ for $R>0$ with $\bar{\Omega} \subset B_{R}$. Moreover, if $G \in C_{0}^{\infty}\left(\bar{\Omega}^{c}\right)^{3}$, then 
$V \in L^{p}\left(\bar{\Omega}^{c}\right)^{3}$ for $p \in(2, \infty), \nabla V \in L^{p}\left(\bar{\Omega}^{c}\right)^{9}$ for $p \in(4 / 3, \infty), D^{2} V \in L^{p}\left(\bar{\Omega}^{c}\right)^{27}$ and $\nabla \Pi \in L^{p}\left(\bar{\Omega}^{c}\right)^{3}$ for $p \in(1, \infty)$.

The inequalities stated in the next theorem are preliminary versions of our resolvent estimates. In these first versions, the unknowns still appear on the right-hand side.

Corollary 7.3 Let $\lambda \in K_{\tau}$ and $G \in C_{0}^{\infty}\left(\bar{\Omega}^{c}\right)^{3}$. Put $V:=\mathfrak{V}(\lambda, G), \Pi:=\Pi(\lambda, G)$. Then $V \in C^{\infty}\left(\bar{\Omega}^{c}\right)^{3}$ and $\Pi \in C^{\infty}\left(\bar{\Omega}^{c}\right)$. There is $c_{0} \in \mathbb{C}$ such that $\Pi+c_{0} \in L^{p}\left(\bar{\Omega}^{c}\right)$ for $p \in$ $(3 / 2, \infty)$. Abbreviate $B:=B_{S+1} \backslash \overline{B_{S}}, \mathfrak{K}_{q}(G, V, \Pi):=\|G\|_{q}+\left\|\nabla V\left|\Omega_{S+1}\left\|_{q}+\right\| \Pi\right| \Omega_{S+1}\right\|_{q}$ for $q \in(1, \infty)$. Then $\|V\|_{p} \leq \mathfrak{C}(p, q) \mathfrak{K}_{q}(G, V, \Pi)$ for $q \in(1,2), p \in\left((1 / q-1 / 2)^{-1}, \infty\right)$ if $q \geq 3 / 2, p \in\left((1 / q-1 / 2)^{-1},(1 / q-2 / 3)^{-1}\right)$ if $q<3 / 2$, and $p=6$ if $q=6 / 5$. Moreover $\|\nabla V\|_{p} \leq \mathfrak{C}\left(p, q, c_{0}\right) \mathfrak{K}_{q}(G, V, \Pi)$ for $q \in(1,3], \quad p \in\left((1 / q-1 / 4)^{-1},(1 / q-\right.$ $\left.1 / 3)^{-1}\right)$, and $p=2$ if $q=6 / 5$. Furthermore $\left\|D^{2} V \mid \Omega_{R}\right\|_{q} \leq \mathfrak{C}(q, R) \mathfrak{K}_{q}(G, V, \Pi)$ for $q \in$ $(1, \infty), R \in(0, \infty)$ with $\bar{\Omega} \subset B_{R}$, and $\left\|D^{2} V\right\|_{2} \leq \mathfrak{C} \mathfrak{K}_{2}(G, V, \Pi)$. In addition, if $\lambda \neq 0$, we have $|\lambda|^{2+4(-1 / q+1 / p)}\|V\|_{p} \leq \mathfrak{C}(p, q) \mathfrak{K}_{q}(G, V, \Pi)$ for $q \in(1,2], p \in[q, 2]$. We further have $\|\nabla \Pi\|_{q} \leq \mathfrak{C}(q) \mathfrak{K}_{q}(G, V, \Pi)$ for $q \in(1, \infty)$. Finally

$$
\|\nabla V\|_{2}+\left\|\Pi+c_{0}\right\|_{2} \leq \mathfrak{C}\left(\left\|G\left|\bar{\Omega}^{c}\left\|_{-1,2}+\right\| V\right| B\right\|_{2}+\|\Pi \mid B\|_{-1,2}\right),
$$

with $c_{0}$ as above, and

$$
\|V\|_{p} \leq \mathfrak{C}(p)\left(\left\|G\left|\bar{\Omega}^{c}\left\|_{-1,2}+\right\| V\right| B\right\|_{2}+\|\Pi \mid B\|_{-1,2}\right) \quad \text { for } p \in(4,6) .
$$

Proof: By Corollary 4.4, we have $V \in C^{\infty}\left(\bar{\Omega}^{c}\right)^{3}$ and $\Pi \in C^{\infty}\left(\bar{\Omega}^{c}\right)$. Moreover Corollary 4.3 yields

$$
V\left|\Omega_{R} \in W^{2, p}\left(\Omega_{R}\right)^{3}, \Pi\right| \Omega_{R} \in W^{1, p}\left(\Omega_{R}\right) \quad \text { for } p \in(1, \infty), R \in(0, \infty) \text { with } \bar{\Omega} \subset B_{R} \text {. (7.3) } \mathbf{C 7 . 3 0 . 1 0 0}
$$

Recall that $B=B_{S+1} \backslash \overline{B_{S}}$. Choose some function $\varphi \in C_{0}^{\infty}\left(\mathbb{R}^{3}\right)$ satisfying the relations $0 \leq \varphi \leq 1, \varphi\left|B_{S+1 / 4}=1, \varphi\right| B_{S+3 / 4}^{c}=0$. Then $\nabla \varphi \cdot V \in C_{0}^{\infty}(B)$. Recalling that the pair $(V, \Pi)$ is a solution to (1.18), we note that $V \mid \partial \Omega=0$ and $\operatorname{div} V=0$, so $\int_{B} \nabla \cdot V d x=0$. Therefore we may apply Theorem 3.7 , setting $\mathfrak{D}:=\mathfrak{D}(2,1, S, S+1)(\nabla \varphi \cdot V \mid B)$. This means in particular that $\mathfrak{D} \in C_{0}^{\infty}(B)^{3}$. Moreover, taking account of the last statement of Theorem 3.7, we get

$$
\|\mathfrak{D}\|_{2, p} \leq C(p)\|\nabla \varphi \cdot V\|_{1, p} \leq C(p)\|V \mid B\|_{1, p} \text { for } p \in(1, \infty) .
$$

Let $\widetilde{V}, \widetilde{\Pi}, H$ denote the zero extension to $\mathbb{R}^{3}$ of $(1-\varphi) V+\mathfrak{D},(1-\varphi) \Pi$ and $2 \sum_{k=1}^{3} \partial_{k} \varphi \partial_{k} V$ $+\Delta \varphi V-\tau \partial_{1} \varphi V-\Delta \mathfrak{D}+\tau \partial_{1} \mathfrak{D}+\lambda \mathfrak{D}-\Pi \nabla \varphi$, respectively. By Theorem 3.7 and the choice of $\mathfrak{D}$, we have $\operatorname{div} \widetilde{V}=0$. Thus the pair $(\widetilde{V}, \widetilde{\Pi})$ solves $(4.5)$ with $V, \Pi, G$ replaced by $\widetilde{V}, \widetilde{\Pi}$ and $(1-\varphi) G+H$, respectively. In view of Corollary 7.1 and 7.2 , we know that $V \in L^{p}\left(\bar{\Omega}^{c}\right)^{3}$ and $\nabla V \in L^{r}\left(\bar{\Omega}^{c}\right)^{9}$ for certain $p, r \in(1, \infty)$. Therefore, and because of the relations $\mathfrak{D} \in C_{0}^{\infty}(B)^{3}$ and (7.3), an analogous property is true for $\widetilde{V}$ and $\nabla \widetilde{V}$, respectively. On the other hand, since $(1-\varphi) G+H \in C_{0}^{\infty}\left(\mathbb{R}^{3}\right)^{3}$, the functions $\bar{V}:=E^{(\lambda)} * P_{2}((1-\varphi) G+H), \bar{\Pi}:=Q_{2}((1-\varphi) G+H)$, satisfy all the properties listed in Corollary 5.3, with $\bar{V}, \bar{\Pi},(1-\varphi) G+H$ in the place of $V, \Pi, G$, respectively. Thus we may apply Theorem 6.1 to obtain $\widetilde{V}=\bar{V}$. Corollary 3.2 then implies there is $c_{0} \in \mathbb{C}$ with $\widetilde{\Pi}+c_{0}=\bar{\Pi}$. In view of the choice of $\varphi$, and because $\mathfrak{D} \in C_{0}^{\infty}(B)^{3}$, we may conclude that

$$
V\left|B_{S+1}^{c}=\widetilde{V}\right| B_{S+1}^{c}=\bar{V}\left|B_{S+1}^{c}, \quad \Pi+c_{0}\right| B_{S+1}^{c}=\widetilde{\Pi}+c_{0}\left|B_{S+1}^{c}=\bar{\Pi}\right| B_{S+1}^{c} .
$$


From (7.3), (7.5) and the fact that $\bar{\Pi}$ possesses all the properties stated for $\Pi$ in Corollary 5.3 , we may conclude that $\Pi+c_{0} \in L^{p}\left(\bar{\Omega}^{c}\right)$ for $p \in(3 / 2, \infty)$, which is the first claim of Corollary 7.3. Due to (7.4), we get

$$
\|H\|_{p} \leq \mathfrak{C}(p)\left(\left\|V\left|\left\|_{1, p}+\right\| \Pi\right| B\right\|_{p}\right) \quad \text { for } p \in(1, \infty) .
$$

Since $V \mid \partial \Omega=0$, Poincaré's inequality applied on $\Omega_{S+1}$ yields

$$
\|G\|_{p}+\left\|V\left|B\left\|_{1, p}+\right\| \Pi\right| B\right\|_{p} \leq \mathfrak{C}(p) \mathfrak{K}_{p}(G, V, \Pi) \text { for } p \in(1, \infty) .
$$

At this point we observe that the functions $\bar{V}$ and $\bar{\Pi}$ may be estimated by applying Corollary 5.1 with $G$ replaced by $(1-\varphi) G+H$, and then referring to (7.6) and (7.7). Taking account of the equations $\bar{V}=\widetilde{V}$ and $\bar{\Pi}=\widetilde{\Pi}+c_{0}$, we thus see that the inequalities in Corollary 7.3 up to but excluding (7.1), with $\widetilde{V}, \widetilde{\Pi}$ in the role of $V$ and $\Pi$, respectively, follow from Corollary 5.1. Put $W:=\varphi V-\mathfrak{D}, \Gamma:=\varphi \Pi$. Then $W \in C^{\infty}\left(\bar{\Omega}^{c}\right)^{3}, \Gamma \in C^{\infty}\left(\bar{\Omega}^{c}\right)$, and the equations in (4.5) hold with $V, \Pi, G$ replaced by $W, \Gamma, \varphi G-H$, respectively. From (7.3), we conclude that $W \mid \Omega_{S+1} \in W^{2, q}\left(\Omega_{S+1}\right)^{3}$ and $\Pi \mid \Omega_{S+1} \in W^{1, q}\left(\Omega_{S+1}\right)$ for any $q \in(1, \infty)$. Moreover, since $\mathfrak{D} \in C_{0}^{\infty}(B)^{3}$ and by the choice of $\varphi$ and $G$, we have $\varphi G-H \in$ $C_{0}^{\infty}\left(\Omega_{S+1}\right)^{3}, W\left|B_{S+3 / 4}^{c}=0, \Gamma\right| B_{S+3 / 4}^{c}=0$ and $W|\partial \Omega=V| \partial \Omega=0$. This means in particular that $W \mid \Omega_{S+1} \in W_{0}^{1, q}\left(\Omega_{S+1}\right)^{3}$ for any $q \in(1, \infty)$. Thus we see that we may apply Theorem 4.6, in particular the estimate at the end of this theorem, as well as Corollary 4.6 with $A=\Omega_{S+1}$ and with $V, \Pi, G$ replaced by $W\left|\Omega_{S+1}, \Gamma\right| \Omega_{S+1}, \varphi G-H \mid \Omega_{S+1}$, respectively. Again taking account of (7.6) and (7.7), and recalling that $W$ and $\Gamma$ vanish outside $B_{S+1}$, we see that the first, second and fifth (if $\lambda \neq 0$ ) inequality in Corollary 7.3 with $V$ replaced by $W$ on the left-hand side follow from Corollary 4.6. Inequalities (7.6) and (7.7) and the estimate at the end of Theorem 4.6 yield that $\left\|D^{2} W\right\|_{q}+\|\nabla \Pi\|_{q}$ is bounded by $\mathfrak{C}(q) \mathfrak{K}_{q}(G, V, \Pi)$ for $q \in(1, \infty)$. This means that the third, forth and sixth estimate in Corollary 7.3 hold with $V, \Pi$ replaced by $W, \Gamma$, respectively, on the left-hand side. Since $V=\widetilde{V}+W, \Pi=\widetilde{\Pi}+c_{0}+\Gamma$, we have thus proved the estimates up to but excluding (7.1) in Corollary 7.3.

In order to derive (7.1) and (7.2), take $\gamma \in C_{0}^{\infty}\left(\mathbb{R}^{3}\right)^{3}$. With (3.1), we get $\|\gamma \mid B\|_{2} \leq$ $\mathfrak{C}\left\|\gamma\left|B\left\|_{6} \leq \mathfrak{C}\right\| \gamma\right|{\overline{B_{S}}}^{c}\right\|_{6} \leq \mathfrak{C}\|\nabla \gamma\|_{2}$. Moreover, by Theorem 3.7, in particular by its last statement, we obtain $\|\mathfrak{D}\|_{1,2} \leq \mathfrak{C}\|\nabla \varphi \cdot V\|_{2} \leq\|V \mid B\|_{2}$. Using these estimates, as well as the relations $\mathfrak{D} \in C_{0}^{\infty}(B)^{3}$ and $\operatorname{supp}(\nabla \varphi) \subset B$, we get

$$
\begin{aligned}
& \left|\int_{\mathbb{R}^{3}} \Delta \mathfrak{D} \cdot \gamma d x\right| \leq \mathfrak{C}\|\nabla \mathfrak{D}\|_{2}\|\nabla \gamma\|_{2} \leq \mathfrak{C}\|V \mid B\|_{2}\|\nabla \gamma\|_{2}, \\
& \left|\lambda \int_{\mathbb{R}^{3}} \mathfrak{D} \cdot \gamma d x\right| \leq \mathfrak{C}\|\mathfrak{D}\|_{2}\left\|\gamma\left|B\left\|_{2} \leq \mathfrak{C}\right\| V\right| B\right\|_{2}\|\nabla \gamma\|_{2}, \\
& \left|\int_{\mathbb{R}^{3}} \sum_{k=1}^{3} \partial_{k} \varphi \partial_{k} V \cdot \gamma d x\right|=\left|\int_{\mathbb{R}^{3}}\left(\Delta \varphi \gamma+\sum_{k=1}^{3} \partial_{k} \varphi \partial_{k} \gamma\right) \cdot V d x\right| \leq \mathfrak{C}\|V \mid B\|_{2}\|\nabla \gamma\|_{2}, \\
& \left|\int_{\mathbb{R}^{3}} \Pi \nabla \varphi \cdot \gamma d x\right| \leq\left\|\Pi\left|B\left\|_{-1,2}\right\| \nabla \varphi \cdot \gamma\left\|_{1,2} \leq \mathfrak{C}\right\| \Pi\right| B\right\|_{-1,2}\|\nabla \gamma\|_{2} .
\end{aligned}
$$

It is obvious how to handle the remaining terms of the integral $\int_{\mathbb{R}^{3}} H \cdot \gamma d x$. Thus, collecting the preceding estimates, we arrive at the inequality $\|H\|_{-1,2} \leq \mathfrak{C}\left(\left\|V\left|B\left\|_{2}+\right\| \Pi\right| B\right\|_{-1,2}\right)$. 
Noting that $\operatorname{supp}(1-\varphi) \subset{\overline{B_{S+1 / 4}}}^{c}$ and $\bar{\Omega} \subset B_{S}$, we obtain $(1-\varphi) \gamma \in C_{0}^{\infty}\left(\bar{\Omega}^{c}\right)^{3}$ for $\gamma \in C_{0}^{\infty}\left(\mathbb{R}^{3}\right)^{3}$. On recalling that $\operatorname{supp}(\nabla \varphi) \subset B$ and $\|\gamma \mid B\|_{2} \leq \mathfrak{C}\|\nabla \gamma\|_{2}$, we further find that $\|\nabla((1-\varphi) \gamma)\|_{2} \leq \mathfrak{C}\|\nabla \gamma\|_{2}$. As a consequence $\|(1-\varphi) G\|_{-1,2} \leq \mathfrak{C}\left\|G \mid \bar{\Omega}^{c}\right\|_{-1,2}$. In view of the estimate of $\|H\|_{-1,2}$ given further above, this means

$$
\|(1-\varphi) G+H\|_{-1,2} \leq \mathfrak{C}\left(\left\|G\left|\bar{\Omega}^{c}\left\|_{-1,2}+\right\| V\left\|_{2}+\right\| \Pi\right| B\right\|_{-1,2}\right) .
$$

Next we remark that the functions $\bar{V}$ and $\bar{\Pi}$ may be estimated by making use of Theorem 5.3 and Lemma 5.1 with $G$ replaced by $(1-\varphi) G+H$. Again recalling that $\bar{V}=\widetilde{V}$ and $\widetilde{\Pi}=\widetilde{\Pi}+c_{0}$, we may then conclude that inequalities (7.1) and (7.2) with $V$, $\Pi$ replaced by $\widetilde{V}, \widetilde{\Pi}$, respectively, follow from Theorem 5.3, Lemma 5.1 and (7.8).

In order to estimate $W$ and $\Gamma$, we fix a function $\zeta \in C_{0}^{\infty}(B)$ with $\zeta \geq 0$ and $\int_{B} \zeta d x>0$. Put $c_{\zeta}:=\left(\int_{B} \zeta d x\right)^{-1} \int_{B} \Gamma \zeta d x$. Corollary 4.5 with $A=\Omega_{S+1}$ and with $G$ replaced by $\varphi G-H \mid \Omega_{S+1}$ imply that

$$
\|W\|_{1,2}+\|W\|_{p}+\left\|\Gamma-c_{\zeta}\left|\Omega_{S+1}\left\|_{2} \leq \mathfrak{C}(p)\right\| \varphi G-H\right| \Omega_{S+1}\right\|_{-1,2} \quad \text { for } p \in(4,6) .
$$

Obviously $\left\|\varphi G-H\left|\Omega_{S+1}\left\|_{-1,2} \leq\right\| \varphi G-H\right| \bar{\Omega}^{c}\right\|_{-1,2}$, so we may conclude with the estimate of $\|H\|_{-1,2}$ we derived above that for $p \in(4,6)$,

$$
\|W\|_{1,2}+\|W\|_{p}+\left\|\Gamma-c_{\zeta} \mid \Omega_{S+1}\right\|_{2} \leq \mathfrak{C}(p)\left(\left\|G\left|\bar{\Omega}^{c}\left\|_{-1,2}+\right\| V\right| B\right\|_{2}+\|\Pi \mid B\|_{-1,2}\right) .
$$

Since $V=\widetilde{V}+W, \Pi=\widetilde{\Pi}+c_{0}+\Gamma$, we arrive at inequalities (7.1) and (7.2), but we still have to add the term $\mathfrak{C} \cdot\left\|c_{\zeta} \mid \Omega_{S+1}\right\|_{2}$ on the right-hand side of (7.1). However, since $\zeta \in C_{0}^{\infty}(B)$, hence $\varphi \zeta \in C_{0}^{\infty}(B)$, we get with Poincaré's inequality on $B$ and the definition of $\Gamma$ that

$$
\left\|c_{\zeta}\left|\Omega_{S+1}\left\|_{2} \leq \mathfrak{C}\left|c_{\zeta}\right| \leq \mathfrak{C}\left|\int_{B} \Pi \varphi \zeta d x\right| \leq \mathfrak{C}\right\| \Pi\right| B\right\|_{-1,2}\|\varphi \zeta\|_{1,2} \leq \mathfrak{C}\|\Pi \mid B\|_{-1,2} .
$$

In the last inequality, we subsumed the term $\|\varphi \zeta\|_{1,2}$ in the constant $\mathfrak{C}$. Estimate (7.9) completes the proof of (7.1).

The ensuing two theorems are the key elements of the proof of our resolvent estimates.

Theorem 7.1 Let $\lambda \in K_{\tau}, q \in(1,2), G \in L^{q}\left(\bar{\Omega}^{c}\right)^{3}$. Then $\|\mathfrak{V}(\lambda, G)\|_{p_{1}} \leq \mathfrak{C}\left(p_{1}, q\right)\|G\|_{q}$ for $p_{1} \in\left((1 / q-1 / 2)^{-1}, \infty\right)$ if $q \geq 3 / 2, p_{1} \in\left((1 / q-1 / 2)^{-1},(1 / q-2 / 3)^{-1}\right)$ in the case $q<3 / 2$, and $p_{1}=6$ if $q=6 / 5$. Moreover $\|\nabla \mathfrak{V}(\lambda, G)\|_{p_{2}} \leq \mathfrak{C}\left(p_{2}, q\right)\|G\|_{q}$ for $p_{2} \in\left((1 / q-1 / 4)^{-1},(1 / q-1 / 3)^{-1}\right)$, and $p_{2}=2$ if $q=6 / 5$. In addition, if $\lambda \neq 0$, we have $|\lambda|^{2+4(-1 / q+1 / p)}\|\mathfrak{V}(\lambda, G)\|_{p_{3}} \leq \mathfrak{C}\left({ }_{3}, q\right)\|G\|_{q}$ for $p_{3} \in[q, 2]$. Finally $\left\|D^{2} \mathfrak{V}(\lambda, G) \mid \Omega_{R}\right\|_{q} \leq$ $\mathfrak{C}(q, R)\|G\|_{q}$ for $q \in(1, \infty), R \in(0, \infty)$ with $\bar{\Omega} \subset B_{R}$, and $\|\nabla \Pi(\lambda, G)\|_{q} \leq \mathfrak{C}(q)\|G\|_{q}$ for $q \in(1, \infty)$.

Proof: We proceed by contradiction, similarly to the approach by Kozono, Sohr [33] and Borchers, Sohr [5]. Take $p_{1}, p_{2}, p_{3}$ as in the theorem, and let $R \in[S+1, \infty)$. Abbreviate $\kappa:=2+4\left(-1 / q+1 / p_{3}\right)$. Suppose there is no constant $C_{0}>$ such that

$$
\begin{gathered}
\|\mathfrak{V}(\varrho, \phi)\|_{p_{1}}+\|\nabla \mathfrak{V}(\varrho, \phi)\|_{p_{2}}+\sup _{T>0}|\varrho|^{\kappa}\left\|\chi_{B_{T}} \mathfrak{V}(\varrho, \phi)\right\|_{p_{3}}+\left\|D^{2} \mathfrak{V}(\varrho, \phi) \mid \Omega_{R}\right\|_{q} \\
+\|\nabla \Pi(\varrho, \phi)\|_{q} \leq C_{0}\|\phi\|_{q} \quad \text { for } \phi \in C_{0}^{\infty}\left(\bar{\Omega}^{c}\right)^{3}, \varrho \in K_{\tau} .
\end{gathered}
$$


Then, for any $n \in \mathbb{N}$, there is some $\varrho_{n} \in K_{\tau}$ and some function $\phi_{n} \in C_{0}^{\infty}\left(\bar{\Omega}^{c}\right)^{3}$ with $\gamma_{n} \geq$ $n\left\|\phi_{n}\right\|_{q}$, where $\gamma_{n}:=\left\|\mathfrak{V}\left(\varrho_{n}, \phi_{n}\right)\right\|_{p_{1}}+\left\|\nabla \mathfrak{V}\left(\varrho_{n}, \phi_{n}\right)\right\|_{p_{2}}+\sup _{T>0}\left|\varrho_{n}\right|^{\kappa}\left\|\chi_{B_{T}} \mathfrak{V}\left(\varrho_{n}, \phi_{n}\right)\right\|_{p_{3}}+$ $\left\|D^{2} \mathfrak{V}\left(\varrho_{n}, \phi_{n}\right) \mid \Omega_{R}\right\|_{q}+\left\|\nabla \Pi\left(\varrho_{n}, \phi_{n}\right)\right\|_{q}$. Note that by the regularity properties listed in Corollary $7.1\left(\varrho_{n} \neq 0\right)$ or $(7.2)\left(\varrho_{n}=0\right)$, we have $\gamma_{n}<\infty$ for $n \in \mathbb{N}$. This is true even if $\varrho_{n}=0$ for some $n \in \mathbb{N}$, in which case the function $\mathfrak{V}\left(\varrho_{n}, \phi_{n}\right)$ need not belong to $L^{p_{3}}\left(\bar{\Omega}^{c}\right)^{3}$. However the term $\sup _{T>0}\left|\varrho_{n}\right|^{\kappa}\left\|\chi_{B_{T}} \mathfrak{V}\left(\varrho_{n}, \phi_{n}\right)\right\|_{p_{3}}$ then vanishes. We do not use the expression $\left|\varrho_{n}\right|^{\kappa}\left\|\mathfrak{V}\left(\varrho_{n}, \phi_{n}\right)\right\|_{p_{3}}$ for $\varrho \in K_{\tau}, \phi \in C_{0}^{\infty}\left(\bar{\Omega}^{c}\right)^{3}$ because in the case $\varrho=0$, it might lead to products of the form $0 \cdot \infty$, which we want to avoid. Define $v_{n}:=\gamma_{n}^{-1} \mathfrak{V}\left(\varrho_{n}, \phi_{n}\right), \sigma_{n}:=\gamma_{n}^{-1} \Pi\left(\varrho_{n}, \phi_{n}\right), g_{n}:=\gamma_{n}^{-1} \phi_{n}$ for $n \in \mathbb{N}$. Then Corollary $7.1-$ 7.3 yield $v_{n} \in C^{\infty}\left(\bar{\Omega}^{c}\right)^{3}, \sigma_{n} \in C^{\infty}\left(\bar{\Omega}^{c}\right)^{3}, v_{n} \mid \Omega_{R} \in W^{2, q}\left(\Omega_{R}\right)^{3}$,

$$
-\Delta v_{n}+\tau \partial_{1} v_{n}+\varrho_{n} v_{n}+\nabla \sigma_{n}=g_{n}, \quad \operatorname{div} v_{n}=0, \quad v_{n} \mid \partial \Omega=0 \quad \text { for } n \in \mathbb{N} .
$$

Since $\gamma_{n} \geq n\left\|\phi_{n}\right\|_{q}$, we further get

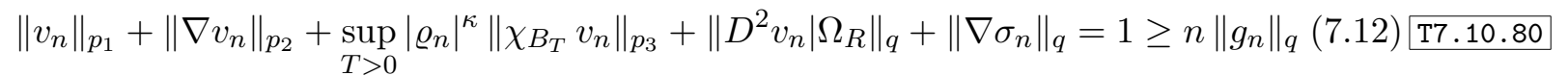

for $n \in \mathbb{N}$. As a first consequence of (7.12), we note that $\left\|g_{n}\right\|_{q} \rightarrow 0$. By the choice of $\Pi\left(\varrho_{n}, \phi_{n}\right)$ (Corollary 7.1 and 7.2), we have $\int_{\Omega_{S+1}} \sigma_{n} d x=0$, hence by a Poincaré's inequality $\left\|\sigma_{n}\left|\Omega_{S+1}\left\|_{q} \leq \mathfrak{C}(q)\right\| \nabla \sigma_{n}\right| \Omega_{S+1}\right\|_{q} \leq \mathfrak{C}(q)\left\|\nabla \sigma_{n}\right\|_{q}$ for $n \in \mathbb{N}$. Thus we may conclude from (7.12) that the sequence $\left(\sigma_{n} \mid \Omega_{S+1}\right)_{n \geq 1}$ is bounded in $W^{1, q}\left(\Omega_{S+1}\right)$. Since $p_{1} \geq q, p_{2} \geq q, R \geq S+1$, it further follows from (7.12) that the sequencee $\left(v_{n} \mid \Omega_{S+1}\right)_{n \geq 1}$ is bounded in $W^{2, q}\left(\Omega_{S+1}\right)^{3}$. We may conclude from (7.12) and from the preceding remarks on $\left(\sigma_{n} \mid \Omega_{S+1}\right)_{n \geq 1}$ that there is a subsequence of $\left(\left(v_{n}, \sigma_{n}, \varrho_{n}\right)\right)$, also denoted by $\left(\left(v_{n}, \sigma_{n}, \varrho_{n}\right)\right)$, with the following properties: $v_{n} \rightarrow V$ in $L^{p_{1}}\left(\bar{\Omega}^{c}\right)^{3}$ for some $V \in L^{p_{1}}\left(\bar{\Omega}^{c}\right)^{3}, \partial_{l} v_{n} \rightarrow V^{(l)}$ in $L^{p_{2}}\left(\bar{\Omega}^{c}\right)^{3}$ for some $V^{(l)} \in L^{p_{2}}\left(\bar{\Omega}^{c}\right)^{3}(1 \leq l \leq 3), \nabla \sigma_{n} \rightarrow \gamma$ in $L^{q}\left(\bar{\Omega}^{c}\right)^{3}$ for some $\gamma \in L^{q}\left(\bar{\Omega}^{c}\right)^{3}$, $\left\|v_{n} \mid \Omega_{S+1}-Z\right\|_{1, q} \rightarrow 0$ for some $Z \in W^{1, q}\left(\Omega_{S+1}\right)^{3},\left\|\sigma_{n} \mid \Omega_{S+1}-Y\right\|_{q} \rightarrow 0$ for some $Y \in$ $L^{q}\left(\Omega_{S+1}\right)^{3}$, and $\varrho_{n} \rightarrow \varrho$ in $\mathbb{C}$ for some $\varrho \in K_{\tau}$. By considering the sequences $\left(\int_{\bar{\Omega}^{c}} v_{n} \cdot \partial_{l} \psi d x\right)$ and $\left(\int_{\bar{\Omega}^{c}} \partial_{l} v_{n} \cdot \psi d x\right)$ with $l \in\{1,2,3\}, \psi \in C_{0}^{\infty}\left(\bar{\Omega}^{c}\right)^{3}$, we find that $V \in W_{l o c}^{1,1}\left(\bar{\Omega}^{c}\right)^{3}$ and $\partial_{l} V=V^{(l)}$ for $1 \leq l \leq 3$. Similarly we get $V \mid \Omega_{S+1}=Z, \quad Y \in W_{l o c}^{1,1}\left(\Omega_{S+1}\right), \quad \nabla Y=$ $\gamma \mid \Omega_{S+1}$. Since $\partial_{l} V=V^{(l)}(1 \leq l \leq 3)$, we conclude that $\nabla V \in L^{p_{2}}\left(\bar{\Omega}^{c}\right)^{9}$. The equation $\int_{\Omega_{S+1}} \sigma_{n} d x=0$ for $n \in \mathbb{N}$ and the fact that $\left\|\sigma_{n} \mid \Omega_{S+1}-Y\right\|_{q} \rightarrow 0$ yield $\int_{\Omega_{S+1}} Y d x=0$. We further conclude that $\left\|v_{n}-V \mid \Omega_{S+1}\right\|_{1, q} \rightarrow 0$, so $V \mid \partial \Omega=0$ by (7.11). The latter reference, the relation $\left\|g_{n}\right\|_{q} \rightarrow 0$ and the equation $\partial_{l} V=V^{(l)}(1 \leq l \leq 3)$ imply that $\operatorname{div} V=0$ and that (4.1) as well as (4.2) hold with $A=\bar{\Omega}^{c}, G=0, \lambda=\varrho$, and with the term $-\Pi \operatorname{div} \psi$ in (4.2) replaced by $\gamma \cdot \psi$. Recalling that $V \in L^{p_{1}}\left(\bar{\Omega}^{c}\right)^{3}, \nabla V \in L^{p_{2}}\left(\bar{\Omega}^{c}\right)^{9}$, we may now apply Theorem 6.2 to obtain $V=0$. Thus (4.2) reduces to the equation $\int \bar{\Omega}^{c} \gamma \cdot \psi d x=0$ for $\psi \in C_{0}^{\infty}\left(\bar{\Omega}^{c}\right)^{3}$, which means that $\gamma=0$. Since $\nabla Y=\gamma \mid \Omega_{S+1}$ and $\int_{\Omega_{S+1}} Y d x=0$, as mentioned above, we get $Y=0$. In this way we arrive at the relations $\left\|v_{n} \mid \Omega_{S+1}\right\|_{1, q} \rightarrow 0$ and $\left\|\sigma_{n} \mid \Omega_{S+1}\right\|_{q} \rightarrow 0$. On the other hand, referring to Corollary 7.3, with $V, \Pi$ replaced by $v_{n}=\mathfrak{V}\left(\varrho_{n}, \gamma_{n}^{-1} \phi_{n}\right), \sigma_{n}=\Pi\left(\varrho_{n}, \gamma_{n}^{-1} \phi_{n}\right)$, we see that the left-hand side of (7.12) is bounded by a constant times $\left\|g_{n}\right\|_{q}+\left\|v_{n}\left|\Omega_{S+1}\left\|_{1, q}+\right\| \sigma_{n}\right| \Omega_{S+1}\right\|_{q}$, uniformly in $n \in \mathbb{N}$. But by what we have found before, this latter term tends to zero for $n \rightarrow \infty$. Thus the left-hand side of (7.12) must equally tend to zero, which is a contradiction. So we have shown there is $C_{0}>0$ such that inequality (7.10) holds for $\phi \in C_{0}^{\infty}\left(\bar{\Omega}^{c}\right)^{3}$, uniformly in $\varrho \in K_{\tau}$. In order to extend this result to $G \in L^{q}\left(\bar{\Omega}^{c}\right)^{3}$, fix some such function $G$, and 
let $\left(\phi_{n}\right)$ be a sequence in $C_{0}^{\infty}\left(\bar{\Omega}^{c}\right)^{3}$ with $\left\|G-\phi_{n}\right\|_{q} \rightarrow 0$. Let $\varrho \in K_{\tau}$. If $\varrho \neq 0$, then the estimate in Theorem 4.9 yields $\left\|V-V_{n}\right\|_{2, q} \rightarrow 0$ and $\left\|\nabla \Pi-\nabla \Pi_{n}\right\|_{q} \rightarrow 0$, where we used the abbreviations $V=\mathfrak{V}(\varrho, G), \Pi=\Pi(\varrho, G), V_{n}=\mathfrak{V}\left(\varrho, \phi_{n}\right)$ and $\Pi_{n}=\Pi\left(\varrho_{n}, \phi_{n}\right)$, for $n \in \mathbb{N}$. If $\varrho=0$, we use Theorem 4.7 to obtain $\left\|V-V_{n}\right\|_{2 q /(2-q)} \rightarrow 0,\left\|\nabla\left(V-V_{n}\right)\right\|_{4 q /(4-q)} \rightarrow$ $0,\left\|D^{2}\left(V-V_{n}\right)\right\|_{q} \rightarrow 0$ and $\left\|\nabla\left(\Pi-\Pi_{n}\right)\right\|_{q} \rightarrow 0$. On the other hand, since inequality (7.10) was shown to be valid for $\phi \in C_{0}^{\infty}\left(\bar{\Omega}^{c}\right)^{3}$, we see that the sequence $\left(V_{n}\right)$ converges in $L^{p_{1}}\left(\bar{\Omega}^{c}\right)^{3},\left(\nabla V_{n}\right)$ in $L^{p_{2}}\left(\bar{\Omega}^{c}\right)^{9},\left(|\varrho|^{\kappa} V_{n}\right)$ in $L^{p_{3}}\left(\bar{\Omega}^{c}\right)^{3}$ if $\varrho \neq 0,\left(D^{2} V_{n} \mid \Omega_{R}\right)$ in $L^{q}\left(\Omega_{R}\right)^{27}$ and $\left(\nabla \Pi_{n}\right)$ in $L^{q}\left(\bar{\Omega}^{c}\right)^{3}$. Since $L^{p}$-convergence implies pointwise convergence a. e. of a subsequence, we may now conclude that the limit functions of the preceding sequences are $V, \nabla V,|\varrho|^{\kappa} V, D^{2} V \mid \Omega_{R}$ and $\nabla \Pi$, respectively. Thus inequality (7.10) remains valid when $\phi$ is replaced by $G$. This proves the theorem.

Theorem 7.2 Let $p \in(4,6), \lambda \in K_{\tau}$ and $G \in C_{0}^{\infty}\left(\bar{\Omega}^{c}\right)^{3}$. Then, for some $c_{0} \in \mathbb{R}$, we have

$$
\|\mathfrak{V}(\lambda, G)\|_{p}+\|\nabla \mathfrak{V}(\lambda, G)\|_{2}+\left\|\Pi(\lambda, G)+c_{0}\right\|_{2} \leq \mathfrak{C}(p)\left\|G \mid \bar{\Omega}^{c}\right\|_{-1,2} .
$$

Proof: We again proceed by contradiction. Suppose there is no constant $C_{0}>0$ such that

$$
\|\mathfrak{V}(\varrho, \phi)\|_{p}+\|\nabla \mathfrak{V}(\varrho, \phi)\|_{2}+\|\Pi(\varrho, \phi)+c(\varrho, \phi)\|_{2} \leq C_{0}\left\|\phi \mid \bar{\Omega}^{c}\right\|_{-1,2}
$$

for $\varrho \in K_{\tau}, \phi \in C_{0}^{\infty}\left(\bar{\Omega}^{c}\right)^{3}$, where $c(\varrho, \phi) \in \mathbb{C}$ is chosen in such a way that $\Pi(\varrho, \phi)+c(\varrho, \phi) \in$ $L^{r}\left(\bar{\Omega}^{c}\right)$ for any $r \in(3 / 2, \infty)$; see Corollary 7.3. Then, for any $n \in \mathbb{N}$, there exists $\varrho_{n} \in K_{\tau}$ and $\phi_{n} \in C_{0}^{\infty}\left(\bar{\Omega}^{c}\right)^{3}$ such that $\gamma_{n} \geq n\left\|\phi_{n} \mid \bar{\Omega}^{c}\right\|_{-1,2}$, where $\gamma_{n}:=\left\|\mathfrak{V}\left(\varrho_{n}, \phi_{n}\right)\right\|_{p}+$ $\left\|\nabla \mathfrak{V}\left(\varrho_{n}, \phi_{n}\right)\right\|_{2}+\left\|\Pi\left(\varrho_{n}, \phi_{n}\right)+c\left(\varrho_{n}, \phi_{n}\right)\right\|_{2}$. Note that $\gamma_{n}<\infty$ for $n \in \mathbb{N}$ by the choice of $c\left(\varrho_{n}, \phi_{n}\right)$ and by Corollary 7.1 and 7.2. We define

$$
v_{n}:=\gamma_{n}^{-1} \mathfrak{V}\left(\varrho_{n}, \phi_{n}\right), \sigma_{n}:=\gamma_{n}^{-1}\left(\Pi\left(\varrho_{n}, \phi_{n}\right)+c\left(\varrho_{n}, \phi_{n}\right)\right), g_{n}:=\gamma_{n}^{-1} \phi_{n} \quad \text { for } n \in \mathbb{N} .
$$

Then, as in the proof of Theorem 7.1, the pair $\left(v_{n}, \varrho_{n}\right)$ satisfies $(7.11)(n \in \mathbb{N})$. The regularity properties listed in Corollary 7.1 in the case $\varrho_{n} \neq 0$ and in Corollary 7.2 if $\varrho_{n}=0$ are valid for $v_{n}, \sigma_{n}$ in the place of $V$ and $\Pi$, respectively, and the first claim in Corollary 7.3 holds with $\Pi=\sigma_{n}$ and $c_{0}=0$. Moreover Corollary 7.3 yields $v_{n, j}, \sigma_{n} \in C_{0}^{\infty}\left(\bar{\Omega}^{c}\right)^{3}$ for $n \in \mathbb{N}, 1 \leq j \leq 3$. The inequality $\gamma_{n} \geq n\left\|\phi_{n} \mid \bar{\Omega}^{x}\right\|_{-1,2}$ implies that

$$
\left\|v_{n}\right\|_{p}+\left\|\nabla v_{n}\right\|_{2}+\left\|\sigma_{n}\right\|_{2}=1 \geq n\left\|g_{n} \mid \bar{\Omega}^{c}\right\|_{-1,2} \quad \text { for } n \in \mathbb{N} .
$$

As a first consequence of (7.15), we note that $\left\|g_{n} \mid \bar{\Omega}^{c}\right\|_{-1,2} \rightarrow 0$. Fix some function $\zeta \in C_{0}^{\infty}\left(B_{S+1}\right)$ with $\zeta \mid B_{S}=1$. Put $B_{S+1} \backslash \overline{B_{S}}$. Since $v_{n} \mid \partial \Omega=0$ according to (7.11), and because $p>2, v_{n} \in L^{p}\left(\bar{\Omega}^{c}\right)^{3}, \nabla v_{n} \in L^{2}\left(\bar{\Omega}^{c}\right)^{9}$, it follows that $\zeta v_{n} \mid \Omega_{S+1} \in W_{0}^{1,2}\left(\Omega_{S+1}\right)^{3}$ and $\left\|\zeta v_{n} \mid \Omega_{S+1}\right\|_{1,2} \leq \mathfrak{C}(p)\left(\left\|v_{n}\right\|_{p}+\left\|\nabla v_{n}\right\|_{2}\right)$, and similarly $\left\|v_{n} \mid \Omega_{S+1}\right\|_{1,2} \leq \mathfrak{C}(p)\left(\left\|v_{n}\right\|_{p}+\right.$ $\left.\left\|\nabla v_{n}\right\|_{2}\right)$, for $n \in \mathbb{N}$. These observations, (7.15) and Theorem 3.3 allow us to choose a subsequence of $\left(\left(v_{n}, \sigma_{n}, \varrho_{n}\right)\right)$, also denoted by $\left(\left(v_{n}, \sigma_{n}, \varrho_{n}\right)\right)$, with the following properties: $v_{n} \rightarrow V$ in $L^{p}\left(\bar{\Omega}^{c}\right)^{3}$ for some $V \in L^{p}\left(\bar{\Omega}^{c}\right)^{3}, \partial_{l} v_{n} \rightarrow V^{(l)}$ in $L^{2}\left(\bar{\Omega}^{c}\right)^{3}$ for some $V^{(l)} \in L^{2}\left(\bar{\Omega}^{c}\right)^{3}(1 \leq l \leq 3), \zeta v_{n} \mid \Omega_{S+1} \rightarrow Z$ in $W_{0}^{1,2}\left(\Omega_{S+1}\right)^{3}$ for some $Z \in W_{0}^{1,2}\left(\Omega_{S+1}\right)^{3}$, $\left\|v_{n} \mid B-Y\right\|_{1,2} \rightarrow 0$ for some $Y \in W^{1,2}(B)^{3}, \sigma_{n} \rightarrow \Pi$ in $L^{2}\left(\bar{\Omega}^{c}\right)^{3}$ for some $\Pi \in L^{2}\left(\bar{\Omega}^{c}\right)^{3}$, $\left\|\sigma_{n} \mid B-\Gamma\right\|_{-1,2} \rightarrow 0$ for some $\Gamma \in L^{2}(B)$, and $\varrho_{n} \rightarrow \varrho$ in $\mathbb{C}$ for some $\varrho \in K_{\tau}$. The relation $\left\|\sigma_{n} \mid B-\Gamma\right\|_{-1,2} \rightarrow 0$ for some $\Gamma \in L^{2}(B)$ is a consequence of Theorem 3.3. By the reasoning 
indicated in the proof of Theorem 7.1, we may conclude that $V \in W_{l o c}^{1,1}\left(\bar{\Omega}^{c}\right)^{3}, \partial_{l} V=V^{(l)}$ for $1 \leq l \leq 3, \zeta V\left|\Omega_{S+1}=Z, V\right| B=Y, \Pi \mid B=\Gamma$. Therefore $\nabla V \in L^{2}\left(\bar{\Omega}^{c}\right)^{9}, \| v_{n}-$ $V \mid B \|_{2} \rightarrow 0$ and $\left\|\sigma_{n}-\Pi \mid B\right\|_{-1,2} \rightarrow 0$. Since $\zeta V\left|\Omega_{S+1}=Z \in W_{0}^{1,2}\left(\Omega_{S+1}\right)^{3}, \zeta\right| B_{S}=1$ and $\bar{\Omega} \subset B_{S}$, we get $V \mid \partial \Omega=0$. Moreover, recalling that $\partial_{l} V=V^{(l)}$ for $1 \leq l \leq 3$ and $\left\|g_{n} \mid \bar{\Omega}^{c}\right\|_{-1,2} \rightarrow 0$, we may deduce from (7.11) that $\operatorname{div} V=0$ and that (4.1) as well as (4.2) hold with $A=\bar{\Omega}^{c}, G=0$ and $\lambda=\varrho$. Since $V \in L^{p}\left(\bar{\Omega}^{c}\right)^{3}$ and $\nabla V \in L^{2}\left(\bar{\Omega}^{c}\right)^{9}$, we may at this point apply Theorem 6.2 to obtain $V=0$. Now (4.2) yields $\int \bar{\Omega}^{c} \Pi \operatorname{div} \psi=0$ for $\psi \in C_{0}^{\infty}\left(\bar{\Omega}^{c}\right)^{3}$, implying that $\Pi \in W_{l o c}^{1,1}\left(\bar{\Omega}^{c}\right)^{3}$ with $\nabla \Pi=0$. As a consequence $\Pi=c$ a. e. for some $c \in \mathbb{C}$ (Corollary 3.2). But $\Pi \in L^{2}\left(\bar{\Omega}^{c}\right)$, so $\Pi=0$, in particular $\Gamma=0$. In this way we arrive at the relations $\left\|v_{n} \mid B\right\|_{2} \rightarrow 0$ and $\left\|\sigma_{n} \mid B\right\|_{-1,2} \rightarrow 0$. On the other hand $\sigma_{n} \in L^{2}\left(\bar{\Omega}^{c}\right)$ by the choice of that function. Thus we may apply (7.1) and (7.2) with $V, \Pi$ replaced by $v_{n}=\mathfrak{V}\left(\varrho_{n}, \gamma_{n}^{-1} \phi_{n}\right), \varrho_{n}=\Pi\left(\varrho_{n}, \gamma_{n}^{-1} \phi_{n}\right)+\gamma_{n}^{-1} c\left(\varrho_{n}, \phi_{n}\right)$, to obtain $\left\|v_{n}\right\|_{p}+\left\|\nabla v_{n}\right\|_{2}+\left\|\sigma_{n}\right\|_{2} \leq \mathfrak{C}(p)\left(\left\|g_{n}\left|\bar{\Omega}^{c}\left\|_{-1,2}+\right\| v_{n}\right| B\right\|_{2}+\left\|\varrho_{n} \mid B\right\|_{-1,2}\right)$ for $n \in \mathbb{N}$. It follows that the left-hand side of this estimate tends to zero for $n \rightarrow \infty$, which is a contradiction to (7.15). As a consequence, there must be a constant $C_{0}>0$ such that (7.14) holds. This proves Theorem 7.2 .

Theorem 7.3 Let $\lambda \in K_{\tau}$ and $G \in \mathcal{D}_{0}^{-1,2}\left(\bar{\Omega}^{c}\right)^{3}$. Then there is a unique function $V:=$ $\widetilde{\mathfrak{V}}(\lambda, G) \in \mathcal{D}_{0}^{1,2}\left(\bar{\Omega}^{c}\right)^{3}$ such that $\operatorname{div} V=0$,

$$
\int_{\bar{\Omega}^{c}}\left(\nabla V \cdot \nabla \psi+\tau \partial_{1} V \psi+\lambda V \cdot \psi\right) d x=G(\psi) \quad \text { for } \psi \in C_{0}^{\infty}\left(\bar{\Omega}^{c}\right)^{3} \text { with div } \psi=0 . \text { (7.16) T7.40.10 }
$$

This function $V$ satisfies the inequality $\|V\|_{p}+\|\nabla V\|_{2} \leq \mathfrak{C}(p)\|G\|_{-1,2}$ for $p \in(4,6]$.

Proof: Since $\mathcal{D}_{0}^{1,2}\left(\bar{\Omega}^{c}\right)^{3} \subset L^{6}\left(\bar{\Omega}^{c}\right)^{3}, \nabla W \in L^{2}\left(\bar{\Omega}^{c}\right)^{9}$ and $W \mid \partial \Omega=0$ for $W \in \mathcal{D}_{0}^{1,2}\left(\bar{\Omega}^{c}\right)^{3}$, the uniqueness statement in Theorem 7.3 follows from Theorem 6.2. Concerning existence, Theorem 3.6 yields a sequence $\left(\phi_{n}\right)$ in $C_{0}^{\infty}\left(\bar{\Omega}^{c}\right)^{3}$ with $\left\|G-\phi_{n}\right\|_{-1,2} \rightarrow 0$. Therefore, by (7.13), the sequence $\left(\mathfrak{V}\left(\lambda, \phi_{n}\right)\right)$ converges in $L^{6}\left(\bar{\Omega}^{c}\right)^{3}$, and the sequence $\left(\nabla \mathfrak{V}\left(\lambda, \phi_{n}\right)\right)$ in $L^{2}\left(\bar{\Omega}^{c}\right)^{9}$. Thus there is a function $V:=\widetilde{\mathfrak{V}}(\lambda, G) \in L^{6}\left(\bar{\Omega}^{c}\right)^{3} \cap W_{l o c}^{1,1}\left(\bar{\Omega}^{c}\right)^{3}$ with $\nabla V \in L^{2}\left(\bar{\Omega}^{c}\right)^{9}$ such that $\left\|V-\mathfrak{V}\left(\lambda, \phi_{n}\right)\right\|_{6} \rightarrow 0$ and $\left\|\nabla V-\nabla \mathfrak{V}\left(\lambda, \phi_{n}\right)\right\|_{2} \rightarrow 0$. Then $\left\|V-\mathfrak{V}\left(\lambda, \phi_{n}\right) \mid \Omega_{S}\right\|_{1,2} \rightarrow$ 0 , so $V \mid \partial \Omega=0$. We thus have found that $V \in \mathcal{D}_{0}^{1,2}\left(\bar{\Omega}^{c}\right)^{3}$. The relations $\left\|V-\mathfrak{V}\left(\lambda, \phi_{n}\right)\right\|_{6} \rightarrow$ 0 and $\left\|\nabla V-\nabla \mathfrak{V}\left(\lambda, \phi_{n}\right)\right\|_{2} \rightarrow 0$ and the fact that $\mathfrak{V}\left(\lambda, \phi_{n}\right)$ satisfies (1.18) with $\phi_{n}$ in the place of $G$ imply (7.16). Again referring to (7.13), we get $\mathfrak{V}\left(\lambda, \phi_{n}\right)\left\|_{p}+\right\| \nabla \mathfrak{V}\left(\lambda, \phi_{n}\right) \|_{2} \leq$ $\mathfrak{C}\left\|\phi_{n} \mid \bar{\Omega}^{c}\right\|_{-1,2}$ for $n \in \mathbb{N}$ and $p \in(4,6)$. In view of the estimate in Theorem 3.5, the preceding inequality also holds for $p=6$. These observations imply the estimate stated in Theorem 7.3.

Theorem 7.4 For $p \in(1, \infty)$, the estimate $\|\mathfrak{V}(\lambda, G)\|_{p} \leq \mathfrak{C}(p)|\lambda|^{-2}\|G\|_{p}$ holds for $G \in$ $L^{p}\left(\bar{\Omega}^{c}\right)^{3}, \lambda \in K_{\tau} \backslash\{0\}$.

Proof: Let $\lambda \in K_{\tau} \backslash\{0\}$. If $p \in(1, \infty), G \in L^{p}\left(\bar{\Omega}^{c}\right)^{3}$, we write $\mathfrak{V}^{+}(\lambda, G)$ for the solution of (1.18) if $\mu=1$, and $\mathfrak{V}^{-}(\lambda, G)$ in the case $\mu=-1$. The notation $\Pi^{+}(\lambda, G)$ and $\Pi^{-}(\lambda, G)$ are to be understood in the same way. For $p \in(1, \infty)$, put $D_{p}:=H_{p}\left(\bar{\Omega}^{c}\right) \cap W^{2, p}\left(\bar{\Omega}^{c}\right)^{3} \cap$ $W_{0}^{1, p}\left(\bar{\Omega}^{c}\right)^{3}, \mathfrak{A}_{p}^{ \pm}(V):=\mathcal{P}_{p}\left(-\Delta V \pm \tau \partial_{1} V+\lambda V\right)$ for $V \in D_{p}$, with $\mathcal{P}_{p}$ introduced in Theorem 2.1. Let $p \in(1, \infty), G \in H_{p}\left(\bar{\Omega}^{c}\right)$, and abbreviate $V:=\mathfrak{V}^{ \pm}(\lambda, G), \Pi:=\Pi^{ \pm}(\lambda, G)$. Then $V \in W^{2, p}\left(\bar{\Omega}^{c}\right)^{3}$, and the pair $(V, \Pi)$ is a solution of (1.18) with $\mu= \pm 1$; see Corollary 7.1. In particular we have $V \mid \partial \Omega=0$ (hence $V \in W_{0}^{1, p}\left(\bar{\Omega}^{c}\right)^{3}$ ) and $\operatorname{div} V=0$. Thus Theorem 
3.2 implies that $V \in H_{p}\left(\bar{\Omega}^{c}\right)$. These observations mean that $V \in D_{p}$. Since $\nabla \Pi \in L^{p}\left(\bar{\Omega}^{c}\right)^{3}$ and the pair $(V, \Pi)$ solves (1.18) with $\mu= \pm 1$. (Corollary 7.1), it follows with the third equation in Corollary 3.7 that $\mathfrak{A}_{p}^{ \pm}(V)=\mathcal{P}_{p}(G)$. Since $G \in H_{p}\left(\bar{\Omega}^{c}\right)$, this means $\mathfrak{A}_{p}^{ \pm}(V)=G$. We have thus shown that $\mathfrak{A}_{p}^{ \pm}: D_{p} \mapsto H_{p}\left(\bar{\Omega}^{c}\right)$ is onto, with

$$
\mathfrak{A}_{p}^{ \pm}\left(\mathfrak{V}^{ \pm}(\lambda, G)\right)=G \quad \text { for } G \in H_{p}\left(\bar{\Omega}^{c}\right) .
$$

Let $V \in D_{p}$ with $\mathfrak{A}_{p}^{ \pm}(V)=0$. Obviously $-\Delta V \pm \tau \partial_{1} V+\lambda V \in L^{p}\left(\bar{\Omega}^{c}\right)^{3}$, so we may put $\Pi:=-\mathcal{G}_{p}\left(-\Delta V \pm \tau \partial_{1} V+\lambda V\right)$. Note that $\Pi \in W_{l o c}^{1, p}\left(\bar{\Omega}^{c}\right)$ with $\nabla \Pi \in L^{p}\left(\bar{\Omega}^{c}\right)^{3}$ by Theorem 2.1. This latter reference and the assumption $\mathfrak{A}_{p}^{ \pm}(V)=0$ imply the pair $(V, \Pi)$ is a solution of (4.5) with $\mu= \pm 1$ and $G=0$. Since $V \in D_{p} \subset W_{0}^{1, p}\left(\bar{\Omega}^{c}\right)^{3}$, we additionally have $V \mid \partial \Omega=0$, so the pair is a solution of (1.18) with $G=0, \mu= \pm 1$. Theorem 6.2 now yields $V=0$. Thus the operator $\mathfrak{A}_{p}^{ \pm}$is one-to-one. At this point we have found that $\mathfrak{A}_{p}^{ \pm}: D_{p} \mapsto H_{p}\left(\bar{\Omega}^{c}\right)$ is bijective, with (7.17) implying

$$
\left(\mathfrak{A}_{p}^{ \pm}\right)^{-1}(G)=\mathfrak{V}^{ \pm}(\lambda, G) \text { for } G \in H_{p}\left(\bar{\Omega}^{c}\right) .
$$

This latter equation, the fact that $\mathfrak{V}^{ \pm}(\lambda, G) \in D_{p}$, and Theorem 4.9 yield that $\left(\mathfrak{A}_{p}^{ \pm}\right)^{-1}$ : $H_{p}\left(\bar{\Omega}^{c}\right) \mapsto H_{p}\left(\bar{\Omega}^{c}\right)$ is bounded.

Next we observe that by integrations by parts and the second equation in Corollary 3.7, the equation $\int_{\bar{\Omega}^{c}} \mathfrak{A}_{p}^{+}(V) \cdot W d x=\int_{\bar{\Omega}} V \cdot \mathfrak{A}_{p}^{-}(W) d x$ holds for $V \in D_{p}, W \in D_{p^{\prime}}$, and for $p \in(1, \infty)$. It follows again with the second equation in Corollary 3.7 that the operator $\left(\mathfrak{A}_{p^{\prime}}^{-}\right)^{-1} \circ \mathcal{P}_{p^{\prime}}$ is dual in $L^{p}\left(\bar{\Omega}^{c}\right)^{3}$ to $\left(\mathfrak{A}_{p}^{+}\right)^{-1} \circ \mathcal{P}_{p}(p \in(1, \infty))$.

Let the operator norm of linear bounded operators $\mathfrak{F}: L^{p}\left(\bar{\Omega}^{c}\right)^{3} \mapsto L^{p}\left(\bar{\Omega}^{c}\right)^{3}$ be denoted by $\left\||\||_{p}\right.$, that is, $\| \mid \mathfrak{F} \|_{p}:=\sup \left\{\|\mathfrak{F}(V)\|_{p} /\|V\|_{p}: V \in L^{p}\left(\bar{\Omega}^{c}\right)^{3}, V \neq 0\right\}$, for $p \in((1, \infty)$. Then a functional analytical principle allows us to deduce from the preceding result that

$$
\left.\left\|\left|\left(\mathfrak{A}_{p}^{+}\right)^{-1} \circ \mathcal{P}_{p}\left\|\left.\right|_{p}=\right\|\right|\left(\mathfrak{A}_{p^{\prime}}^{-}\right)^{-1} \circ \mathcal{P}_{p^{\prime}}\right\|\right|_{p^{\prime}} \quad(p \in(1, \infty)) .
$$

Now let $p \in(2, \infty)$. Then $p^{\prime} \in(1,2)$, so by Corollary 7.3 we know that $\left\|\mathfrak{V}^{-}(\lambda, G)\right\|_{p^{\prime}} \leq$ $\mathfrak{C}\left(p^{\prime}\right)|\lambda|^{-2}\|G\|_{p^{\prime}}$ for $G \in L^{p^{\prime}}\left(\bar{\Omega}^{c}\right)^{3}$. Using (2.1) and (7.18), we may conclude that $\| \mid\left(\mathfrak{A}_{p^{\prime}}^{-}\right)^{-1} \circ$ $\mathcal{P}_{p^{\prime}} \|\left.\right|_{p^{\prime}} \leq \mathfrak{C}\left(p^{\prime}\right)|\lambda|^{-2}$. In view of (7.19), we thus obtain $\left\|\left.\left|\left(\mathfrak{A}_{p}^{+}\right)^{-1} \circ \mathcal{P}_{p}\|\|_{p} \leq \mathfrak{C}(p)\right| \lambda\right|^{-2}\right.$. Here $p$ was arbitrarily taken from $(2, \infty)$. If $p \in(1,2)$, the preceding inequality follows directly from Corollary 7.3, (7.18) and (2.1). In order to handle the case $p=2$, we interpolate between $L^{3}\left(\bar{\Omega}^{c}\right)^{3}$ and $L^{3 / 2}\left(\bar{\Omega}^{c}\right)^{3}$. To this end, we note that by (7.18) and Lemma 7.2 , we get $\left(\mathfrak{A}_{3}^{+}\right)^{-1}\left(\mathcal{P}_{3}(G)\right)=\mathfrak{V}^{+}\left(\lambda, \mathcal{P}_{3}(G)\right)=\mathfrak{V}^{+}(\lambda, G)=\mathfrak{V}^{+}\left(\lambda, \mathcal{P}_{3 / 2}(G)\right)=$ $\left(\mathfrak{A}_{3 / 2}^{+}\right)^{-1}\left(\mathcal{P}_{3 / 2}(G)\right)$ for $G \in L^{3}\left(\bar{\Omega}^{c}\right)^{3} \cap L^{3 / 2}\left(\bar{\Omega}^{c}\right)^{3}$. Since we showed that the estimate $\left\|\left|\left(\mathfrak{A}_{p}^{+}\right)^{-1} \circ \mathcal{P}_{p} \|\right|_{p} \leq \mathfrak{C}(p)|\lambda|^{-2}\right.$ is valid for $p \in(1, \infty) \backslash\{2\}$, we may now conclude by the Riesz-Thorin interpolation theorem that it holds for $p=2$ as well. Thus we have proved this estimate for any $p \in(1, \infty)$. Combining it with Lemma 7.2, (7.18) and (2.1), we finally get $\left\|\mathfrak{V}^{+}(\lambda, G)\right\|_{p}=\left\|\mathfrak{V}^{+}\left(\lambda, \mathcal{P}_{p}(G)\right)\right\|_{p}=\left(\mathfrak{A}_{p}^{+}\right)^{-1}\left(\mathcal{P}_{p}(G)\right)\left\|_{p} \leq \mathfrak{C}(p)|\lambda|^{-2}\right\| \mathcal{P}_{p}(G) \|_{p} \leq$ $\mathfrak{C}(p)|\lambda|^{-2}\|G\|_{p}$ for $G \in L^{p}\left(\bar{\Omega}^{c}\right)^{3}, p \in(1, \infty)$.

We finish this section by pointing out some technical details about solutions to (1.18). The first is an immediate consequence of Theorem 6.2 and the regularity properties of solutions to (1.18) mentioned in Corollary 7.1, 7.2 and in Theorem 7.3. 
Corollary 7.4 Let $\lambda \in K_{\tau}$ and $p \in(1, \infty)$, with either $\lambda \neq 0$ or $p<2$. Let $G \in$ $L^{p}\left(\bar{\Omega}^{c}\right)^{3} \cap \mathcal{D}_{0}^{-1,2}\left(\bar{\Omega}^{c}\right)^{3}$. Then the function $\mathfrak{V}(\lambda, G)$, as defined in Corollary 7.1 or 7.2, and the function $\widetilde{\mathfrak{V}}(\lambda, G)$ introduced in Theorem 7.3 coincide. Therefore we will use the notation $\mathfrak{V}(\lambda, G)$ instead of $\widetilde{\mathfrak{V}}(\lambda, G)$ also if $\phi \in \mathcal{D}_{0}^{-1,2}\left(\bar{\Omega}^{c}\right)^{3}$.

corollaryC7.60>

Corollary 7.5 Let $p \in(1,2], r \in(1, \min \{p, 6 / 5\})$. Put $\gamma:=(1 / r-1 / 3)^{-1}$. (Note that $\gamma \in(3 / 2,2)$.) Let $\lambda \in K_{\tau} \backslash\{0\}, L \in(0, \infty), \phi \in C_{0}^{\infty}\left(\bar{\Omega}^{c}\right)^{3}$. Then $|\lambda|\left\|\mathfrak{V}\left(\lambda, \chi_{B_{L}} \phi\right)\right\|_{\gamma} \leq$ $\mathfrak{C}(r, L)|\lambda|^{1 / 3}\|\phi\|_{p}$.

Proof: The estimate of the term $|\lambda|^{2+4(-1 / q+1 / p)}\|\mathfrak{V}(\lambda, G)\|_{p_{3}}$ in Theorem 7.1, with $q, p_{3}$ replaced by $r$ and $\gamma$, respectively, implies $|\lambda|\left\|\mathfrak{V}\left(\lambda, \chi_{B_{L}} \phi\right)\right\|_{\gamma} \leq \mathfrak{C}(r)|\lambda|^{1 / 3}\left\|\chi_{B_{L}} \phi\right\|_{r}$. Since $r \leq p$, we further have $\left\|\chi_{B_{L}} \phi\right\|_{r} \leq C(L)\|\phi\|_{p}$.

Corollary 7.6 Let $G \in \mathcal{D}_{0}^{-1,2}\left(\bar{\Omega}^{c}\right)^{3} \cap L^{2}\left(\bar{\Omega}^{c}\right)^{3}$ (hence $\mathfrak{V}(0, G) \in \mathcal{D}_{0}^{1,2}\left(\bar{\Omega}^{c}\right)^{3}$; see Theorem 7.3). Then $\mathfrak{V}(0, G) \in W_{\text {loc }}^{1,1}\left(\bar{\Omega}^{c}\right)^{3}, D^{2} \mathfrak{V}(0, G) \in L^{2}\left(\bar{\Omega}^{c}\right)^{27}, \partial_{1} \mathfrak{V}(0, G) \in \mathcal{D}_{0}^{-1,2}\left(\bar{\Omega}^{c}\right)^{3}$, and there exists a function $\Pi(G) \in W^{1,2}\left(\bar{\Omega}^{c}\right)$ such that (1.18) holds with $V=\mathfrak{V}(0, G), \Pi=$ $\Pi(G)$ and $\lambda=0$.

Proof: By Corollary 3.5, there is a sequence $\left(\phi_{n}\right)$ in $C_{0}^{\infty}\left(\bar{\Omega}^{c}\right)^{3}$ such that $\left\|G-\phi_{n}\right\|_{-1,2} \rightarrow 0$ and $\left\|G-\phi_{n}\right\|_{2} \rightarrow 0$. Then by Theorem 7.3,

$$
\left\|\mathfrak{V}(0, G)-\mathfrak{V}\left(0, \phi_{n}\right)\right\|_{6}+\left\|\nabla\left(\mathfrak{V}(0, G)-\mathfrak{V}\left(0, \phi_{n}\right)\right)\right\|_{2} \rightarrow 0 .
$$

Let $m, n \in \mathbb{N}$. By Corollary 7.3, there are constants $c_{n}, c_{m}, c_{m, n} \in \mathbb{C}$ such that the functions $\Pi\left(0, \phi_{n}\right)+c_{n}, \Pi\left(0, \phi_{m}\right)+c_{m}$ and $\Pi\left(0, \phi_{n}-\phi_{m}\right)+c_{n, m}$ belong to $L^{2}\left(\bar{\Omega}^{c}\right)$. By Corollary 7.2, we know that (1.18) with $\lambda=0$ is satisfied with $\mathfrak{V}\left(0, \phi_{i}\right), \Pi\left(0, \phi_{i}\right)+c_{i}, \phi_{i}$ in the role of $V, \Pi, G$, respectively, for $i \in\{m, n\}$, and also by $\mathfrak{V}\left(0, \phi_{n}-\phi_{m}\right), \Pi\left(0, \phi_{n}-\phi_{m}\right)+$ $c_{n, m}, \phi_{n}-\phi_{m}$ instead of $V, \Pi, G$, respectively. In view of the regularity properties listed in Corollary 7.2, we may now apply Theorem 6.2 and Corollary 3.2 to obtain that $\mathfrak{V}\left(0, \phi_{n}\right)-$ $\mathfrak{V}\left(0, \phi_{m}\right)=\mathfrak{V}\left(0, \phi_{n}-\phi_{m}\right)$, and $\Pi\left(0, \phi_{n}\right)+c_{n}-\left(\Pi\left(0, \phi_{m}\right)+c_{m}\right)=\Pi\left(0, \phi_{n}-\phi_{m}\right)+c_{n, m}$. On the other hand, we have $\left\|\nabla \mathfrak{V}\left(0, \phi_{n}-\phi_{m}\right)\right\|_{2}+\left\|\Pi\left(0, \phi_{n}-\phi_{m}\right)+c_{n, m}\right\|_{2} \leq \mathfrak{C}\left\|\phi_{n}-\phi_{m}\right\|_{-1,2}$ by Theorem 7.2. Moreover $\left\|\nabla\left(\Pi\left(0, \phi_{n}-\phi_{m}\right)+c_{n, m}\right)\right\|_{2} \leq \mathfrak{C}\left\|\phi_{n}-\phi_{m}\right\|_{2}$ according to Theorem 7.1, and the term $\left\|D^{2} \mathfrak{V}\left(0, \phi_{n}-\phi_{m}\right)\right\|_{2}$ is bounded by

$$
\mathfrak{C}\left(\left\|\phi_{n}-\phi_{m}\right\|_{2}+\left\|\nabla \mathfrak{V}\left(0, \phi_{n}-\phi_{m}\right)\left|\Omega_{S+1}\left\|_{2}+\right\| \Pi\left(0, \phi_{n}-\phi_{m}\right)+c_{n, m}\right| \Omega_{S+1}\right\|_{2},\right.
$$

as follows from Corollary 7.3. Bringing all this together, we get

$$
\begin{aligned}
& \left\|D^{2} \mathfrak{V}\left(0, \phi_{n}\right)-D^{2} \mathfrak{V}\left(0, \phi_{m}\right)\right\|_{2}+\left\|\Pi\left(0, \phi_{n}\right)+c_{n}-\left(\Pi\left(0, \phi_{m}\right)+c_{m}\right)\right\|_{1,2} \\
& \leq \mathfrak{C}\left(\left\|\phi_{n}-\phi_{m}\right\|_{2}+\left\|\phi_{n}-\phi_{m}\right\|_{-1,2}\right) .
\end{aligned}
$$

Thus we see that the sequence $\left(D^{2} \mathfrak{V}\left(0, \phi_{n}\right)\right)$ converges in $L^{2}\left(\bar{\Omega}^{c}\right)^{27}$, and the sequence $\left(\Pi\left(0, \phi_{n}\right)+c_{n}\right)$ in $W^{1,2}\left(\bar{\Omega}^{c}\right)$. Let $\Pi(G)$ denote the limit function of that latter sequence. It follows with (7.20) that $\mathfrak{V}(0, G) \in W_{l o c}^{2,1}\left(\bar{\Omega}^{c}\right)^{3}, D^{2} \mathfrak{V}(0, G) \in L^{2}\left(\bar{\Omega}^{c}\right)^{27}$, and $\| D^{2} \mathfrak{V}(0, G)-$ $D^{2} \mathfrak{V}\left(0, \phi_{n}\right) \|_{2} \rightarrow 0$. Thus we may further conclude with (7.20) and Corollary 7.2 that (1.18) is satisfied with $V=\mathfrak{V}(0, G), \Pi=\Pi(G), \lambda=0$. It follows that $\partial_{1} \mathfrak{V}(0, G)=$ $\Delta \mathfrak{V}(0, G)-\nabla \Pi(G)+G$. Since $\partial_{j} \mathfrak{V}(0, G)_{k}, \Pi(G) \in L^{2}\left(\bar{\Omega}^{c}\right)(1 \leq j, k \leq 3)$, it is obvious that $\Delta \mathfrak{V}(0, G)-\nabla \Pi(G)+G \in \mathcal{D}_{0}^{-1,2}\left(\bar{\Omega}^{c}\right)^{3}$, so $\partial_{1} \mathfrak{V}(0, G) \in \mathcal{D}_{0}^{-1,2}\left(\bar{\Omega}^{c}\right)^{3}$. 
${ }^{\langle\text {lemmaL7.20 }}{ }^{20 m m a}$ 7.1 Let $q \in(3 / 2,2), G \in L^{q}\left(\bar{\Omega}^{c}\right)^{3}$. Then $|\mathfrak{V}(0, G)|_{\infty} \leq \mathfrak{C}(q)\|G\|_{q}$.

Proof: Since $2 q /(2-q) \geq q$ and $4 q /(4-q) \geq q$, we may deduce from Corollary 7.2 , Theorem 4.7 and 6.2 that

$$
\begin{aligned}
& \left\|\mathfrak{V}(0, G) \mid \Omega_{S+1}\right\|_{2, q} \leq \mathfrak{C}(q)\left(\|\mathfrak{V}(0, G)\|_{2 q /(2-q)}+\|\nabla \mathfrak{V}(0, G)\|_{4 q /(4-q)}+\left\|D^{2} \mathfrak{V}(0, G)\right\|_{q}\right) \\
& \leq \mathfrak{C}(q)\|G\|_{q} .
\end{aligned}
$$

It follows by a Sobolev inequality that $\left.|\mathfrak{V}(0, G)| \Omega_{S+1}\right|_{\infty} \leq \mathfrak{C}(q)\|G\|_{q}$. If $x \in \bar{\Omega}^{c} \backslash \Omega_{S+1}$, then $\overline{B_{1}(x)} \subset \bar{\Omega}^{c}$, so we obtain by an analogous reasoning that $\left.|\mathfrak{V}(0, G)| B_{1}(x)\right|_{\infty} \leq C(q)\|G\|_{q}$, with a constant $C(q)$ independent of $x$.

$\langle$ lemmaL7.10〉

Lemma 7.2 Let $\lambda \in K_{\tau}, p \in(1,2)$ in the case $\lambda=0, p \in(1, \infty)$ if $\lambda \neq 0, G \in L^{p}\left(\bar{\Omega}^{c}\right)^{3}$. Then $\mathfrak{V}(\lambda, G)=\mathfrak{V}(\lambda, \mathcal{P}(G))$.

Proof: For $\psi \in C_{0}^{\infty}\left(\bar{\Omega}^{c}\right)^{3}$ with $\operatorname{div} \psi=0$, we have $\int_{\bar{\Omega}^{c}} G \cdot \psi d x=\int_{\bar{\Omega}^{c}} \mathcal{P}(G) \cdot \psi d x$ (Theorem 2.1). Therefore (4.5) is satisfied with $V=\mathfrak{V}(\lambda, G)$ and $V=\mathfrak{V}(\lambda, \mathcal{P}(G))$, each time with the function $G$ on the right-hand side. Thus, due to the integrability properties of $\mathfrak{V}(\lambda, G)$ and $\mathfrak{V}(\lambda, \mathcal{P}(G))$ mentioned in Corollary $7.1(\lambda \neq 0)$ and $7.2(\lambda=0)$, we may apply Theorem 6.2 , which yields $\mathfrak{V}(\lambda, G)=\mathfrak{V}(\lambda, \mathcal{P}(G))$.

\section{Resolvent estimates related to the perturbed Oseen prob- lem (1.19).}

We proceed as in the whole space case ([12, Section 4 and 5]). However, a new difficulty arises: In the proof of Theorem 8.1, we need rather precise informations on the asymptotic behaviour of solutions to (1.18) when $|x|$ tends to infinity. In the whole space case, we could exploit in this context that the velocity part of a solution to (4.5) with $A=\mathbb{R}^{3}$ and with $G$ being solenoidal is given explicitly in the form $E^{(\lambda)} * G$. Here, however, we have to rely on a different representation formula (equation (8.5)), due which our argument becomes considerably more involved. This additional difficulty manifests itself in the proof of Theorem 8.1. Otherwise we rather closely follow the reasoning in [12, Section $5-7]$, often referring to the proofs given there.

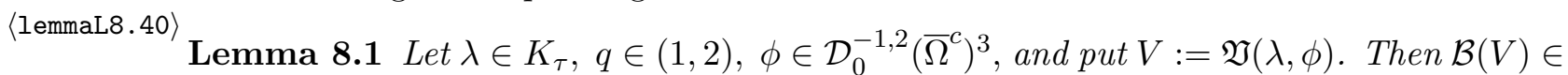
$L^{2}\left(\bar{\Omega}^{c}\right)^{3} \cap L^{q}\left(\bar{\Omega}^{c}\right)^{3}$ and $\mathcal{P}_{2}(\mathcal{B}(V)) \in H_{2}\left(\bar{\Omega}^{c}\right) \cap \mathcal{D}_{0}^{-1,2}\left(\bar{\Omega}^{c}\right)^{3} \cap L^{q}\left(\bar{\Omega}^{c}\right)^{3}$. Let $G \in L^{q}\left(\bar{\Omega}^{c}\right)^{3}$ and set $W:=\mathfrak{V}(\lambda, G)$. Then $\mathcal{B}(W) \in L^{q}\left(\bar{\Omega}^{c}\right)^{3}$. Moreover $\left\|\mathcal{P}_{2}(\mathcal{B}(V))\right\|_{*} \leq \mathfrak{C}\|\phi\|_{-1,2}$ and $\left\|\mathcal{P}_{q}(\mathcal{B}(W))\right\|_{q} \leq \mathfrak{C}(q)\|G\|_{q}$. We further have

$$
\left\|\mathcal{P}_{2}\left(\chi_{B_{R}^{c}} \mathcal{B}(V)\right)\right\|_{*} \leq D_{1}(R)\|\phi\|_{-1,2}, \quad\left\|\mathcal{P}_{q}\left(\chi_{B_{R}^{c}} \mathcal{B}(W)\right)\right\|_{q} \leq D_{2}(q, R)\|G\|_{q}
$$

for $R \in(0, \infty)$, with $D_{1}(R) \rightarrow 0$ and $D_{2}(q, R) \rightarrow 0$ for $R \rightarrow \infty$. (The norm \|\|$_{*}$ was defined at the beginning of Section 2.)

Proof: Let $\widetilde{q} \in\{6 / 5, q, 2\}$. If $\widetilde{q} \geq 4 / 3$ or if $\widetilde{q}<4 / 3$ and $(1 / \widetilde{q}-3 / 4)^{-1}>6$, put $\bar{p}:=6$. In the case $\widetilde{q}<4 / 3$ and $(1 / \widetilde{q}-3 / 4)^{-1} \leq 6$, set $\left.\bar{p}:=\left[\max \{4,1 / \widetilde{q}-1 / 3)^{-1}\right\}+(1 / \widetilde{q}-3 / 4)^{-1}\right] / 2$. 
With this choice of $\bar{p}$, we have $\bar{p} \in(4,6],(1 / \widetilde{q}-1 / \bar{p})^{-1} \in(4 / 3,3]$ and $(1 / \widetilde{q}-1 / 2)^{-1} \in$ $(2, \infty]$; see the proof of $[12$, Lemma 9$]$ for more details. The latter proof now yields Lemma 8.1 if we replace $[12,(2.14)-(2.16)]$ by $(1.5),[12,(3.39),(3.40)]$ by Theorem 7.3 , and [12, Theorem 4] by Theorem 2.1 and the first equation in Corollary 3.7. Also note that $L^{6 / 5}\left(\bar{\Omega}^{c}\right) \subset \mathcal{D}_{0}^{-1,2}\left(\bar{\Omega}^{c}\right)$ (Theorem 3.6). Concerning inequality [12, (4.6)], use Theorem 7.1 instead of $[12,(3.16)$ and $(3.17)]$.

${ }^{\langle\text {lemmaL8. 30 }}$ Lemma 8.2 Let $q \in(1,2), \epsilon>0, G \in L^{q}\left(\bar{\Omega}^{c}\right)^{3}, F \in \mathcal{D}_{0}^{-1,2}\left(\bar{\Omega}^{c}\right)^{3} \cap L^{2}\left(\bar{\Omega}^{c}\right)^{3}$. Then there are functions $\phi, \psi \in C_{0}^{\infty}\left(\bar{\Omega}^{c}\right)^{3}$ such that $\operatorname{div} \phi=0=\operatorname{div} \psi$,

$$
\begin{aligned}
& \left\|\mathcal{P}_{2}\left[\chi_{B_{R}} \mathcal{B}(\mathfrak{V}(\lambda, F)-\mathfrak{V}(\lambda, \phi))\right]\right\|_{*} \leq \epsilon\|F\|_{*}, \quad\left\|\phi \mid \bar{\Omega}^{c}\right\|_{*} \leq 2\|F\|_{*}, \\
& \left\|\mathcal{P}_{q}\left[\chi_{B_{R}} \mathcal{B}(\mathfrak{V}(\lambda, G)-\mathfrak{V}(\lambda, \psi))\right]\right\|_{q} \leq \epsilon\|G\|_{q}, \quad\|\psi\|_{q} \leq 2\|G\|_{q},
\end{aligned}
$$

for $R \in(0, \infty], \lambda \in K_{\tau}$.

Proof: Take $R, \lambda$ as in the lemma. By Theorem 3.13, we know that $\mathcal{P}_{2}(F) \in \mathcal{D}_{0}^{-1,2}\left(\bar{\Omega}^{c}\right)^{3} \cap$ $H_{2}\left(\bar{\Omega}^{c}\right)^{3}$. Therefore by Theorem 3.11 , we may choose a sequence $\left(\phi_{n}\right)$ in $C_{0}^{\infty}\left(\bar{\Omega}^{c}\right)^{3}$ with $\operatorname{div} \phi_{n}=0$ and $\left\|\mathcal{P}_{2}(F)-\phi_{n}\right\|_{*} \leq\|F\|_{*} / n$ for $n \in \mathbb{N}$. Lemma 7.2 states that $\mathfrak{V}(\lambda, F)=$ $\mathfrak{V}\left(\lambda, \mathcal{P}_{2}(F)\right)$. Therefore by Lemma 8.1, for $n \in \mathbb{N}$,

$$
\left\|\mathcal{P}_{2}\left[\chi_{B_{R}} \mathcal{B}\left(\mathfrak{V}(\lambda, F)-\mathfrak{V}\left(\lambda, \phi_{n}\right)\right)\right]\right\|_{*} \leq \mathfrak{C}\left\|\mathcal{P}_{2}(F)-\phi_{n}\right\|_{-1,2} \leq \mathfrak{C}\|F\|_{*} / n .
$$

Since $\mathcal{P}_{q}(G) \in H_{q}\left(\bar{\Omega}^{c}\right)$, we may choose a sequence $\left(\psi_{n}\right)$ in $C_{0}^{\infty}\left(\bar{\Omega}^{c}\right)^{3}$ with $\operatorname{div} \psi_{n}=0$ and $\left\|\mathcal{P}_{q}(G)-\psi_{n}\right\|_{q} \leq \mathfrak{C}\|G\|_{q} / n$ for $n \in \mathbb{N}$. Using Lemma 7.2 and 8.1 as in the proof of (8.1), we further get $\left\|\mathcal{P}_{q}\left[\chi_{B_{R}} \mathcal{B}\left(\mathfrak{V}(\lambda, G)-\mathfrak{V}\left(\lambda, \psi_{n}\right)\right)\right]\right\|_{q} \leq \mathfrak{C}(q)\|G\|_{q} / n$ for $n \in \mathbb{N}$. Lemma 8.2 follows by a suitable choice of $n$.

The next lemma was proved in [12] without being stated explicitly there.

$\langle$ lemmaL8.60〉

Lemma 8.3 Let $\lambda \in K_{\tau} \backslash\{0\}, R, \widetilde{R} \in(0, \infty)$ with $\widetilde{R} \geq 2 R+3, q \in(1,2), \phi \in C_{0}^{\infty}\left(\mathbb{R}^{3}\right)^{3}$. Take some $\gamma \in(3 / 2,2)$, and put $s:=\left[(1 / \gamma-1 / 4)^{-1}+(1 / \gamma-1 / 3)^{-1}\right] / 2$. Then

$$
\begin{aligned}
& \left\|E^{(\lambda)} *\left(\chi_{B_{\widetilde{R}}} \phi\right)-E^{(0)} *\left(\chi_{B_{\widetilde{R}}} \phi\right)\right\|_{\infty}+\left\|\nabla\left(E^{(\lambda)} *\left(\chi_{B_{\widetilde{R}}} \phi\right)-E^{(0)} *\left(\chi_{B_{\widetilde{R}}} \phi\right)\right)\right\|_{s} \\
& \leq \mathfrak{C}(\gamma)|\lambda|^{1 / 3}\left\|\phi \mid B_{\widetilde{R}}\right\|_{1} ; \\
& \left|\int_{B_{\widetilde{R}}^{c}}\left(\partial^{\beta} E^{(\varrho)}\right)(x-y) \phi(y) d y\right| \leq \mathfrak{C}(R)\left[\widetilde{R}^{-1 / 2}+(-\ln (1-1 / \widetilde{R}))^{1 / 2}\right]\|\phi\|_{*}, \\
& \left|\int_{B_{\widetilde{R}}^{c}}\left(\partial^{\beta} E^{(\varrho)}\right)(x-y) \phi(y) d y\right| \leq \mathfrak{C}(q, R) \widetilde{R}^{-1-|\beta| / 2+2 / q^{\prime}}\|\phi\|_{q}
\end{aligned}
$$

for $x \in B_{R+1}, \varrho \in\{0, \lambda\}, \beta \in \mathbb{N}_{0}^{3}$ with $|\beta| \leq 1$.

Proof: See [12, p. 221] for (8.2), and [12, p. 222-223] for (8.3) and (8.4). Note that in $[12,(4.7)]$ and in $[12$, p. $222-223]$, by mistake we wrote $(1 / 2) \ln (\widetilde{R} /(\widetilde{R}-1))$ instead of $\left(\ln (\widetilde{R} /(\widetilde{R}-1))^{1 / 2}\right.$.

$\langle$ theoremT8.10〉 
Theorem 8.1 Let $R, \widetilde{R} \in(0, \infty)$ with $\bar{\Omega} \subset B_{R}, \widetilde{R} \geq 2 R+3$. Let $\lambda \in K_{\tau} \backslash\{0\}, q \in$ $(1,2), G \in C_{0}^{\infty}\left(\bar{\Omega}^{c}\right)^{3}$ with $\operatorname{div} G=0$. Then

$$
\begin{aligned}
& \left\|\mathcal{P}_{2}\left[\chi_{B_{R}} \mathcal{B}(\mathfrak{V}(\lambda, G)-\mathfrak{V}(0, G))\right]\right\|_{*} \\
& \leq\left[\mathfrak{C}(R, \widetilde{R})|\lambda|^{1 / 3}+\mathfrak{C}(R) \widetilde{R}^{-1 / 2}+\mathfrak{C}(R)(-\ln (1-1 / \widetilde{R}))^{1 / 2}\right]\|G\|_{*}, \\
& \left\|\mathcal{P}_{q}\left[\chi_{B_{R}} \mathcal{B}(\mathfrak{V}(\lambda, G)-\mathfrak{V}(0, G))\right]\right\|_{q} \leq\left(\mathfrak{C}(R, \widetilde{R}, q)|\lambda|^{1 / 3}+\mathfrak{C}(R, q) \widetilde{R}^{-1+2 / q^{\prime}}\right)\|G\|_{q} .
\end{aligned}
$$

Proof: Let $\psi \in C_{0}^{\infty}\left(\mathbb{R}^{3}\right)$ with $\psi\left|B_{R}=0, \psi\right| B_{R+1}^{c}=1$. Put $B:=B_{R+1} \backslash \overline{B_{R}}, W^{(\varrho)}:=$ $E^{(\varrho)} * G$ for $\varrho \in\{0, \lambda\}$. Note that in particular $G \in H_{2}\left(\mathbb{R}^{3}\right)$, so $P_{2}(G)=G, Q_{2}(G)=0$ by Theorem 3.12. Let $\varrho \in\{0, \lambda\}$. Since $P_{2}(G)=G \in C^{\infty}\left(\bar{\Omega}^{c}\right)^{3}$, the regularity properties of $W^{(\varrho)}$ correspond to those listed for $V$ in Corollary 5.3. According to Theorem 5.1 and because $Q_{2}(G)=0$, the equations in (4.5) hold with $V=W^{(\varrho)}, \Pi=0$. In particular $\operatorname{div} W^{(\varrho)}=0, \nabla \psi \cdot W^{(\varrho)} \in C_{0}^{\infty}(B)$, so that $\int_{B} \nabla \psi \cdot W^{(\varrho)} d x=0$. Thus the function $\mathfrak{D}^{(\varrho)}:=\mathfrak{D}(2,1, R, R+1)\left(-\nabla \psi \cdot W^{(\varrho)} \mid B\right)$ is well defined and belongs to $C_{0}^{\infty}(B)^{3}$ (Theorem 3.7). Put $Z^{(\varrho)}:=\psi W^{(\varrho)}+\mathfrak{D}^{(\varrho)}$,

$$
F^{(\varrho)}:=-\Delta \psi W^{(\varrho)}-2 \sum_{k=1}^{3} \partial_{k} \psi \partial_{k} W^{(\varrho)}+\tau \partial_{1} \psi W^{(\varrho)}-\Delta \mathfrak{D}^{(\varrho)}+\tau \partial_{1} \mathfrak{D}^{(\varrho)}+\varrho \mathfrak{D}^{(\varrho)} .
$$

Then $F^{(\varrho)} \in C_{0}^{\infty}(B)^{3}$, and the function $Z^{(\varrho)}$ inherits all the properties of $W^{(\varrho)}$, as enumerated in Corollary 5.3 for $V$. Since $B \subset{\overline{B_{R}}}^{c}, \mathfrak{D}^{(\varrho)} \in C_{0}^{\infty}(B)^{3}$ and $\psi \mid \overline{B_{R}}=0$, we have $Z^{(\varrho)} \mid \overline{B_{R}}=0$. There, in view of the definition of $F^{(\varrho)}$, we see that the equations in (1.18) are satisfied with $V, G$ replaced by $Z^{(\varrho)}\left|\bar{\Omega}^{c}, F^{(\varrho)}+\psi G\right| \bar{\Omega}^{c}$, respectively, and with $\Pi=0$. Now put $\widetilde{F}^{(\varrho)}:=-F^{(\varrho)}+(1-\psi) G$. Since $\widetilde{F}^{(\varrho)} \in C_{0}^{\infty}\left(\bar{\Omega}^{c}\right)^{3}$, the regularity properties of $\mathfrak{V}\left(\varrho, \widetilde{F}^{(\varrho)}\right)$ (Corollary 7.1 or 7.2 ) are identical to those listed for $V$ in Corollary 7.3. Moreover (1.18) is satisfied with $V, G, \Pi$ replaced by $\mathfrak{V}\left(\varrho, \widetilde{F}^{(\varrho)}\right), \widetilde{F}^{(\varrho)}, \Pi\left(\varrho, \widetilde{F}^{(\varrho)}\right)$, respectively; see Corollary 7.1 or 7.2 . (The function $\Pi\left(\varrho, \widetilde{F}^{(\varrho)}\right)$ will not be needed here.) In this situation, Theorem 6.2 yields

$$
\mathfrak{V}(\varrho, G)=Z^{(\varrho)}+\mathfrak{V}\left(\varrho, \widetilde{F}^{(\varrho)}\right) \quad \text { for } \varrho \in\{0, \lambda\} .
$$

The preceding equation is the representation formula for $\mathfrak{V}(\varrho, G)$ on which our proof of Theorem 8.1 is based. As mentioned at the beginning of this section, the choice of a suitable representation formula is a difficulty we did not encounter in the whole space case ([12, Theorem 14]). Since the equation $Z^{(\varrho)} \mid B_{R}=0$ implies $\chi_{B_{R}} \mathcal{B}\left(Z^{(\lambda)}-Z^{(0)}\right)=0$, we may deduce from (8.7) that

$$
\begin{aligned}
& \left\|\mathcal{P}_{2}\left[\chi_{B_{R}} \mathcal{B}(\mathfrak{V}(\lambda, G)-\mathfrak{V}(0, G))\right]\right\|_{*}=\left\|\mathcal{P}_{2}\left[\chi_{B_{R}} \mathcal{B}\left(\mathfrak{V}\left(\lambda, \widetilde{F}^{(\lambda)}\right)-\mathfrak{V}\left(0, \widetilde{F}^{(0)}\right)\right)\right]\right\|_{*}(8.8) \text { T8.10.26 } \\
& \leq\left\|\mathcal{P}_{2}\left[\chi_{B_{R}} \mathcal{B}\left(\mathfrak{V}\left(\lambda, \widetilde{F}^{(0)}\right)-\mathfrak{V}\left(0, \widetilde{F}^{(0)}\right)\right)\right]\right\|_{*}+\left\|\mathcal{P}_{2}\left[\chi_{B_{R}} \mathcal{B}\left(\mathfrak{V}\left(\lambda, F^{(\lambda)}-F^{(0)}\right)\right)\right]\right\|_{*} .
\end{aligned}
$$

The equation $\mathfrak{V}\left(\lambda, \widetilde{F}^{(\lambda)}\right)-\mathfrak{V}\left(\lambda, \widetilde{F}^{(0)}\right)=\mathfrak{V}\left(\lambda, \widetilde{F}^{(\lambda)}-\widetilde{F}^{(0)}\right)$ we used here follows from Theorem 6.2. Note that $\widetilde{F}^{(\lambda)}-\widetilde{F}^{(0)}=-F^{(\lambda)}+F^{(0)}$. According to Corollary 7.3, we have $\mathfrak{V}\left(\lambda, \widetilde{F}^{(0)}\right) \in C^{\infty}\left(\bar{\Omega}^{c}\right)^{3} \cap L^{p}\left(\bar{\Omega}^{c}\right)^{3}$ for $p \in(1, \infty)$. So, by taking account of Corollary 7.1 and 7.2 , we may deduce from Theorem 6.2 that

$$
\mathfrak{V}\left(\lambda, \widetilde{F}^{(0)}\right)-\mathfrak{V}\left(0, \widetilde{F}^{(0)}\right)=\mathfrak{V}\left(0,-\lambda \mathfrak{V}\left(\lambda, \widetilde{F}^{(0)}\right)\right) .
$$


Thus with Theorem 3.6 (continuous imbedding of $L^{6 / 5}\left(\bar{\Omega}^{c}\right)$ in $\mathcal{D}_{0}^{-1,2}\left(\bar{\Omega}^{c}\right)$ ), Lemma 8.1 $\left(\mathcal{B}\left(\mathfrak{V}\left(\lambda, \widetilde{F}^{(0)}\right)-\mathfrak{V}\left(0, \widetilde{F}^{(0)}\right)\right) \in L^{p}\left(\bar{\Omega}^{c}\right)^{3}\right.$ for $\left.p \in(1,2]\right)$, the first equation in Corollary 3.7 and (2.1), we arrive at the inequality

$$
\begin{aligned}
& \mathfrak{A}:=\left\|\mathcal{P}_{2}\left[\chi_{B_{R}} \mathcal{B}\left(\mathfrak{V}\left(\lambda, \widetilde{F}^{(0)}\right)-\mathfrak{V}\left(0, \widetilde{F}^{(0)}\right)\right)\right]\right\|_{*} \\
& \leq \mathfrak{C} \sum_{p \in\{6 / 5,2\}}\left\|\chi_{B_{R}} \mathcal{B}\left[\mathfrak{V}\left(0,-\lambda \mathfrak{V}\left(\lambda, \widetilde{F}^{(0)}\right)\right)\right]\right\|_{p} .
\end{aligned}
$$

For $r \in(1,6 / 5)$, we set $\gamma:=\gamma(r):=(1 / r-1 / 3)^{-1}$. Then $\gamma \in(3 / 2,2)$. We further set $s:=s(r):=\left[(1 / \gamma(r)-1 / 4)^{-1}+(1 / \gamma(r)-1 / 3)^{-1}\right] / 2$ for $r \in(1,6 / 5)$. Since $\gamma>3 / 2>$ $4 / 3$, we have $(1 / \gamma-1 / 4)^{-1}>2$, so $s>2 \geq p$ for any $p \in[1,2]$. Choosing $r=11 / 10$, we may deduce from (8.10) that

$$
\begin{aligned}
\mathfrak{A} \leq \mathfrak{C}(R) \sum_{p \in\{6 / 5,2\}}\left(\left\|\nabla U \mid \Omega_{R}\right\|_{p}\left\|\mathfrak{V}\left(0,-\lambda \mathfrak{V}\left(\lambda, \widetilde{F}^{(0)}\right)\right)\right\|_{\infty}\right. \\
\left.+\left\|U \mid \Omega_{R}\right\|_{(1 / p-1 / s)^{-1}}\left\|\nabla \mathfrak{V}\left(0,-\lambda \mathfrak{V}\left(\lambda, \widetilde{F}^{(0)}\right)\right)\right\|_{s}\right) .
\end{aligned}
$$

Here it is important that $s \geq p$ for $p \in\{6 / 5,2\}$. Next we use that $\left\|\nabla U \mid \Omega_{R}\right\|_{p} \leq$ $\mathfrak{C}(R)\|\nabla U\|_{3}$ and $\left\|U \mid \Omega_{R}\right\|_{(1 / p-1 / s)^{-1}} \leq \mathfrak{C}(R)\|U\|_{\infty}$ for $p \in\{6 / 5,2\}$, and we apply Lemma 7.1 and Theorem 7.1, to obtain

$$
\mathfrak{A} \leq \mathfrak{C}(R)|\lambda|\left\|\mathfrak{V}\left(\lambda, \widetilde{F}^{(0)}\right)\right\|_{\gamma} .
$$

Here it is essential that $\gamma \in(3 / 2,2)$. Next, by the choice of $r$ and $\gamma$, by Corollary 7.5 with $p=2$, and because $\operatorname{supp}\left(\widetilde{F}^{(0)}\right) \subset B_{R+1}$, we find $|\lambda|\left\|\mathfrak{V}\left(\lambda, \widetilde{F}^{(0)}\right)\right\|_{\gamma} \leq \mathfrak{C}(R)|\lambda|^{1 / 3}\left\|\widetilde{F}^{(0)}\right\|_{2}$, so that from (8.11),

$$
\mathfrak{A} \leq \mathfrak{C}(R)|\lambda|^{1 / 3}\left\|\widetilde{F}^{(0)}\right\|_{2} .
$$

On the other hand, since $F^{(\lambda)}-F^{(0)} \in C_{0}^{\infty}(B)^{3}$, Lemma 8.1 implies

$$
\begin{aligned}
& \left\|\mathcal{P}_{2}\left[\chi_{B_{R}} \mathcal{B}\left(\mathfrak{V}\left(\lambda, F^{(\lambda)}-F^{(0)}\right)\right)\right]\right\|_{*} \leq \mathfrak{C}(R)\left\|F^{(\lambda)}-F^{(0)} \mid \bar{\Omega}^{c}\right\|_{-1,2} \\
& \leq \mathfrak{C}(R)\left\|F^{(\lambda)}-F^{(0)}\right\|_{2},
\end{aligned}
$$

with the last inequality being valid because $B \subset \Omega_{R+1}$, so $\|\gamma \mid B\|_{2} \leq \mathfrak{C}(R)\|\nabla \gamma\|_{2}$ for $\gamma \in C_{0}^{\infty}\left(\bar{\Omega}^{c}\right)^{3}$ by Poincaré's inequality on $\Omega_{R+1}$. From (8.8), (8.12) and (8.13), we may conclude

$$
\left\|\mathcal{P}_{2}\left[\chi_{B_{R}} \mathcal{B}(\mathfrak{V}(\lambda, G)-\mathfrak{V}(0, G))\right]\right\|_{*} \leq \mathfrak{C}(R)\left(|\lambda|^{1 / 3}\left\|\widetilde{F}^{(0)}\right\|_{2}+\left\|F^{(\lambda)}-F^{(0)}\right\|_{2}\right) .
$$

Let us consider the right-hand side of (8.14). By Theorem 3.7, and because $\nabla \psi \cdot W^{(\varrho)} \in$ $C_{0}^{\infty}(B)^{3}$ for $\varrho \in\{0, \lambda\}$, we get $\left\|\mathfrak{D}^{(\varrho)}\right\|_{2,2} \leq \mathfrak{C}(R)\left\|\nabla \psi \cdot W^{(\varrho)}\right\|_{1,2} \leq \mathfrak{C}\left\|W^{(\varrho)} \mid B\right\|_{1,2}$ and $\left\|\mathfrak{D}^{(\lambda)}-\mathfrak{D}^{(0)}\right\|_{2,2}=\left\|\mathfrak{D}(2,1, R, R+1)\left(-\nabla \psi \cdot\left(W^{(\lambda)}-W^{(0)}\right) \mid B\right)\right\|_{2,2} \leq \mathfrak{C}(R) \| W^{(\lambda)}-$ $\left.W^{(0)}\right) \mid B \|_{1,2}$. Due to these inequalities and some additional, obvious estimates, we find that $\left\|\widetilde{F}^{(0)}\right\|_{2} \leq \mathfrak{C}(R)\left\|W^{(0)} \mid B\right\|_{1,2}+\|G\|_{2}$, and

$$
\left.\left\|F^{(\lambda)}-F^{(0)}\right\|_{2} \leq \mathfrak{C}(R) \| W^{(\lambda)}-W^{(0)}\right) \mid B \|_{1,2} .
$$


Therefore from (8.14),

$$
\begin{aligned}
& \left\|\mathcal{P}_{2}\left[\chi_{B_{R}} \mathcal{B}(\mathfrak{V}(\lambda, G)-\mathfrak{V}(0, G))\right]\right\|_{*} \\
& \leq \mathfrak{C}(R)\left(|\lambda|^{1 / 3}\left\|W^{(0)}\left|B\left\|_{1,2}+|\lambda|^{1 / 3}\right\| G\left\|_{2}+\right\| W^{(\lambda)}-W^{(0)}\right| B\right\|_{1,2}\right) .
\end{aligned}
$$

Thus we have reduced inequality (8.5) to estimates of $W^{(0)} \mid B$ and $W^{(\lambda)}-W^{(0)} \mid B$, similar to the ones arising in the whole space case (Lemma 8.3, [12, proof of Theorem 14]). Since $B \subset B_{R+1}$, we get

$$
\left\|W^{(\lambda)}-W^{(0)} \mid B\right\|_{1,2} \leq C\left(\mathfrak{A}_{1}+\mathfrak{A}_{2}+\mathfrak{A}_{3}\right),
$$

with $\mathfrak{A}_{1}:=\left\|E^{(\lambda)} *\left(\chi_{B_{\widetilde{R}}} G\right)-E^{(0)} *\left(\chi_{B_{\widetilde{R}}} G\right) \mid B_{R+1}\right\|_{2}$,

$$
\begin{aligned}
\mathfrak{A}_{2} & :=\sum_{l=1}^{3}\left\|\left(\partial_{l} E^{(\lambda)}\right) *\left(\chi_{B_{\widetilde{R}}} G\right)-\left(\partial_{l} E^{(0)}\right) *\left(\chi_{B_{\widetilde{R}}} G\right) \mid B_{R+1}\right\|_{2}, \\
\mathfrak{A}_{3} & :=\sum_{\varrho \in\{0, \lambda\}} \sum_{\beta \in \mathbb{N}_{0}^{3},|\beta| \leq 1}\left\|\left(\partial^{\beta} E^{(\lambda)}\right) *\left(\chi_{B_{\widetilde{R}}^{c}} G\right) \mid B_{R+1}\right\|_{2},
\end{aligned}
$$

where we used Theorem 5.1 in the definition of $\mathfrak{A}_{2}$ and $\mathfrak{A}_{3}$. We may estimate $\mathfrak{A}_{1}$ and $\mathfrak{A}_{3}$ in a first step by replacing the $L^{2}$-norm by the $L^{\infty}$-norm, and $\mathfrak{A}_{2}$ by a transition from the $L^{2}$ - to the $L^{s}$-norm, with the exponent $s=s(11 / 10)$ introduced above. (Recall that $s \geq 2$.) This gives rise to a constant $\mathfrak{C}(R)$, which does not matter. Then we evaluate $\mathfrak{A}_{1}+\mathfrak{A}_{2}$ by inequality (8.2), which yields the upper bound $\mathfrak{C}|\lambda|^{1 / 3}\left\|G \mid B_{\widetilde{R}}\right\|_{1}$, and thus $\mathfrak{C}(\widetilde{R})|\lambda|^{1 / 3}\left\|G \mid \bar{\Omega}^{c}\right\|_{*}$. Concerning $\mathfrak{A}_{3}$, we refer to (8.3), and we use Lemma 3.4 in order to estimate $\|G\|_{-1,2}$ by $\mathfrak{C}\left\|G \mid \bar{\Omega}^{c}\right\|_{*}$. In this way, we obtain the upper bound $\mathfrak{C}(R)\left[\widetilde{R}^{-1 / 2}+\right.$ $\left.(-\ln (1-1 / \widetilde{R}))^{1 / 2}\right]\left\|G \mid \bar{\Omega}^{c}\right\| *$. Collecting the preceding estimates from (8.17) onwards, we arrive at the inequality

$$
\begin{aligned}
& \left\|W^{(\lambda)}-W^{(0)} \mid B\right\|_{1,2} \\
& \leq\left[\mathfrak{C}(R, \widetilde{R})|\lambda|^{1 / 3}+\mathfrak{C}(R) \widetilde{R}^{-1 / 2}+\mathfrak{C}(R)(-\ln (1-1 / \widetilde{R}))^{1 / 2}\right]\left\|G \mid \bar{\Omega}^{c}\right\| * .
\end{aligned}
$$

With (3.1), we find that

$$
\left\|W^{(0)}\left|B\left\|_{2} \leq \mathfrak{C}(R)\right\| W^{(0)}\right| B\right\|_{6} \leq \mathfrak{C}(R)\left\|W^{(0)} \mid{\overline{B_{R}}}^{c}\right\|_{6} \leq \mathfrak{C}(R)\left\|\nabla W^{(0)}\right\|_{2} .
$$

But $\left\|\nabla W^{(0)}\right\|_{2} \leq \mathfrak{C}\|G\|_{-1,2}$ by Theorem 5.3 and because $P_{2}(G)=G$, as mentioned above. Again using Lemma 3.4 , we thus get $\left\|W^{(0)}\left|B\left\|_{1,2} \leq \mathfrak{C}(R)\right\| G\right| \bar{\Omega}^{c}\right\|_{*}$. From this estimate, (8.18) and (8.16), we arrive at (8.5).

Concerning (8.6), we start with an analogue of (8.8), that is,

$$
\begin{aligned}
& \left\|\mathcal{P}_{q}\left[\chi_{B_{R}} \mathcal{B}(\mathfrak{V}(\lambda, G)-\mathfrak{V}(0, G))\right]\right\|_{q} \\
& \leq\left\|\mathcal{P}_{q}\left[\chi_{B_{R}} \mathcal{B}\left(\mathfrak{V}\left(\lambda, \widetilde{F}^{(0)}\right)-\mathfrak{V}\left(0, \widetilde{F}^{(0)}\right)\right)\right]\right\|_{q}+\left\|\mathcal{P}_{q}\left[\chi_{B_{R}} \mathcal{B}\left(\mathfrak{V}\left(\lambda, F^{(\lambda)}-F^{(0)}\right)\right)\right]\right\|_{q} .
\end{aligned}
$$

As regards the first term on the right-hand side of the preceding inequality, we first apply (2.1), then use equation (8.9). The second term may be handled by Lemma 8.1, which 
yields the upper bound $\mathfrak{C}(R)\left\|F^{(\lambda)}-F^{(0)}\right\|_{q}$. In this way we get

$$
\begin{aligned}
& \left\|\mathcal{P}_{q}\left[\chi_{B_{R}} \mathcal{B}(\mathfrak{V}(\lambda, G)-\mathfrak{V}(0, G))\right]\right\|_{q} \\
& \leq \mathfrak{C}(q)\left\|\chi_{B_{R}} \mathcal{B}\left[\mathfrak{V}\left(0,-\lambda \mathfrak{V}\left(\lambda, \widetilde{F}^{(0)}\right)\right)\right]\right\|_{q}+\mathfrak{C}(q, R)\left\|F^{(\lambda)}-F^{(0)}\right\|_{q} .
\end{aligned}
$$

In order to evaluate the first term on the right-hand side of (8.19), we choose $r:=(1+$ $\min \{q, 6 / 5\}) / 2$, and then consider the parameters $\gamma=\gamma(r), s=s(r)$ associated with this value of $r$ according to the definitions given above. Proceeding in the same way as in the transition from (8.10) to (8.11), but with $q$ in the role of $p$, we get

$$
\| \chi_{B_{R}} \mathcal{B}\left(\mathfrak{V}\left(0,-\lambda \mathfrak{V}\left(\lambda, \widetilde{F}^{(0)}\right)\right)\left\|_{q} \leq \mathfrak{C}(q, R)|\lambda|\right\| \mathfrak{V}\left(\lambda, \widetilde{F}^{(0)}\right) \|_{\gamma} .\right.
$$

Now we use Corollary 7.5 again, with $q$ in the role of $p$. Due to the preceding choice of $r$, this corollary yields $|\lambda|\left\|\mathfrak{V}\left(\lambda, \widetilde{F}^{(0)}\right)\right\|_{\gamma} \leq \mathfrak{C}(q, R)|\lambda|^{1 / 3}\left\|\widetilde{F}^{(0)}\right\|_{q}$. The preceding estimates from (8.19) onwards lead to the inequality

$$
\left\|\mathcal{P}_{q}\left[\chi_{B_{R}} \mathcal{B}(\mathfrak{V}(\lambda, G)-\mathfrak{V}(0, G))\right]\right\|_{q} \leq \mathfrak{C}(q, R)\left(|\lambda|^{1 / 3}\left\|\widetilde{F}^{(0)}\right\|_{q}+\left\|\widetilde{F}^{(\lambda)}-\widetilde{F}^{(0)}\right\|_{q}\right) \cdot(8.20) \text { T8.10.120 }
$$

Since $\operatorname{supp}\left(\widetilde{F}^{(\lambda)}-\widetilde{F}^{(0)}\right) \subset B \subset B_{R+1}$, and by (8.15) and (8.17), we get $\left\|F^{(\lambda)}-F^{(0)}\right\|_{q} \leq$ $\mathfrak{C}(R)\left\|F^{(\lambda)}-F^{(0)}\right\|_{2} \leq \mathfrak{C}(R)\left(\mathfrak{A}_{1}+\mathfrak{A}_{2}+\mathfrak{A}_{3}\right)$, with $\mathfrak{A}_{1}, \mathfrak{A}_{2}, \mathfrak{A}_{3}$ defined as in the passage following (8.17). In order to evaluate these terms, we again change from the $L^{2}$ - to the $L^{\infty}$ norm $\left(\mathfrak{A}_{1}\right.$ and $\left.\mathfrak{A}_{3}\right)$, or to the $L^{s}$-norm $\left(\mathfrak{A}_{2}\right)$, but with the value of $s=s(r)$ associated with the preceding choice of $r$. On using (8.2) as above, we get $\mathfrak{A}_{1}+\mathfrak{A}_{2} \leq \mathfrak{C}(q)|\lambda|^{1 / 3}\left\|G \mid B_{\widetilde{R}}\right\|_{1}$, then evaluate $\left\|G \mid B_{\widetilde{R}}\right\|_{1}$ by $\mathfrak{C}(\widetilde{R})\|G\|_{q}$. As for $\mathfrak{A}_{3}$, we apply (8.4), obtaining the upper bound $\mathfrak{C}(q, R) \widetilde{R}^{-1+2 / q^{\prime}}\|G\|_{q}$. In this way we arrive at the inequality

$$
\left\|\widetilde{F}^{(\lambda)}-\widetilde{F}^{(0)}\right\|_{q} \leq\left(\mathfrak{C}(R, \widetilde{R})|\lambda|^{1 / 3}+\mathfrak{C}(R) \widetilde{R}^{-1+2 / q^{\prime}}\right)\|G\|_{q} .
$$

Theorem 3.7, in particular its last statement, and the relation $\nabla \psi \cdot W^{(0)} \in C_{0}^{\infty}(B)$, yield that $\left\|\mathfrak{D}^{(\varrho)}\right\|_{2, q} \leq \mathfrak{C}(q, R)\left\|\nabla \psi \cdot W^{(0)}\right\|_{1, q} \leq \mathfrak{C}(q, R)\left\|W^{(\varrho)} \mid B\right\|_{1, q}$. With this inequality available, it is obvious that $\left\|\widetilde{F}^{(0)}\right\|_{q} \leq \mathfrak{C}(q, R)\left\|W^{(0)} \mid B\right\|_{1, q}+\|G\|_{q}$. Now choose $s_{1}:=$ $2(1 / q-1 / 2)^{-1}$ if $q \geq 3 / 2, s_{1}:=\left((1 / q-1 / 2)^{-1}+(1 / q-2 / 3)^{-1}\right) / 2$ if $q<3 / 2, s_{2}:=$ $\left((1 / q-1 / 4)^{-1}+(1 / q-1 / 3)^{-1}\right) / 2$. Then Theorem 5.1 implies

$$
\left\|W^{(0)} \mid B\right\|_{1, q} \leq \mathfrak{C}(R)\left(\left\|W^{(0)}\left|B_{R+1}\left\|_{s_{1}}+\right\| \nabla W^{(0)}\right| B_{R+1}\right\|_{s_{2}}\right) \leq \mathfrak{C}(q, R)\|G\|_{q} .
$$

Therefore $\left\|\widetilde{F}^{(0)}\right\|_{q} \leq \mathfrak{C}(q, R)\|G\|_{q}$, so inequality (8.6) follows from (8.20), (8.21) and the preceding estimate.

Corollary 8.1 Let $R, \widetilde{R}, \lambda$ and $q$ be given as in Theorem 8.1. Then inequality (8.5) holds for $G \in L^{2}\left(\bar{\Omega}^{c}\right)^{3} \cap \mathcal{D}_{0}^{-1,2}\left(\bar{\Omega}^{c}\right)^{3}$, and (8.6) for $G \in L^{q}\left(\bar{\Omega}^{c}\right)^{3}$.

Proof: Suppose that $G \in L^{2}\left(\bar{\Omega}^{c}\right)^{3} \cap \mathcal{D}_{0}^{-1,2}\left(\bar{\Omega}^{c}\right)^{3}$. By Lemma 8.2, we may choose a sequence $\left(G_{n}\right)$ in $C_{0}^{\infty}\left(\bar{\Omega}^{c}\right)^{3}$ with $\operatorname{div} G_{n}=0$ and $\left\|G_{n} \mid \bar{\Omega}^{c}\right\|_{*} \leq 2\|G\|_{*}$ for $n \in \mathbb{N}$, as well as $\left\|\mathcal{P}_{2}\left[\chi_{B_{R}} \mathcal{B}\left(\mathfrak{V}(\varrho, G)-\mathfrak{V}\left(\varrho, G_{n}\right)\right)\right]\right\|_{*} \rightarrow 0(n \rightarrow \infty)$, for $\varrho \in\{0, \lambda\}$. Since $\left\|G_{n} \mid \bar{\Omega}^{c}\right\|_{*} \leq$ $2\|G\|_{*}$, and because Theorem 8.1 implies that inequality (8.5) holds with $G$ replaced by $G_{n}$ for $n \in \mathbb{N}$, we may conclude this latter inequality is even valid for the function $G$ given 
in Corollary 8.1. The same kind of reasoning based on Lemma 8.2 is valid with respect to (8.6).

We recall that the space $\mathcal{D}(\mathcal{L})$ was introduced in $(1.11)$, the operator $\mathcal{B}_{S}$ in $(2.2)$, and the operator $\mathcal{K}$ in $(2.2)$.

$\left\langle\right.$ lemmaL8.70〉 Lemma 8.4 Let $q \in[1,6 / 5]$. Then $\left\|\mathcal{B}_{S}(\phi)\right\|_{q} \leq \mathfrak{C}(q)\|\phi\|_{2}$ for $\phi \in L^{2}\left(\bar{\Omega}^{c}\right)^{3}$. Moreover $\mathcal{B}_{S}(\phi) \in L^{2}\left(\bar{\Omega}^{c}\right)^{3}$ for $\phi \in W^{2,2}\left(\bar{\Omega}^{c}\right)^{3}$.

Proof: See the proof of [12, Lemma 10]; use (1.5) instead of [12, (2.14)].

Theorem 8.2 Let $\xi \in \mathbb{R}$, and let $\sigma \in[0, \infty)$ be an eigenvalue of the operator $\mathcal{K}+\xi \mathcal{P}_{2} \circ \mathcal{B}_{S}$, and $G$ an associated eigenfunction (in particular $G \in H_{2}^{\prime} \subset \mathcal{D}(\mathcal{L})$; see the passage following Theorem 2.2). Then $G \in W^{2, q}\left(\bar{\Omega}^{c}\right)^{3}$ for $q \in(1,6 / 5]$, and $\|\nabla G\|_{2} \leq \mathfrak{C}(\xi)\|G\|_{2},\|G\|_{2, q} \leq$ $\mathfrak{C}(\xi, \sigma, q)\|G\|_{2}$ for $q \in(1,6 / 5]$.

Proof: Since $G \in \mathcal{D}(\mathcal{L})$, the estimate of $\|\nabla G\|_{2}$ follows by the same variational argument as in the whole space case; see [12, proof of Theorem 15], with Lemma 8.4 in the role of [12, Lemma 10], inequality (3.1) in that of $[12,(2.10)]$, and Theorem 2.1 and Corollary 3.7 in the one of $\left[12\right.$, Theorem 4]. Observing that $-\Delta G-\xi \mathcal{B}_{S}(G) \in L^{2}\left(\bar{\Omega}^{c}\right)^{3}$ (Lemma 8.4), we may consider $\Pi:=\mathcal{G}_{2}\left(-\Delta G-\xi \mathcal{B}_{S}(G)\right)$. Then Theorem 2.1 and the equation $\left(\mathcal{K}+\xi \mathcal{P}_{2} \circ \mathcal{B}_{S}\right)(G)=\sigma G$ yield $\Pi \in W_{l o c}^{1,2}\left(\bar{\Omega}^{c}\right), \nabla \Pi \in L^{2}\left(\bar{\Omega}^{c}\right)^{3},-\Delta G+\sigma G+\nabla \Pi=\xi \mathcal{B}_{S}(G)$. The equation $\operatorname{div} G=0$ follows from Lemma 3.2. Thus Lemma 8.4 and Theorem 4.1 imply $G \in W^{2, q}\left(\bar{\Omega}^{c}\right)^{3}$ and $\|G\|_{2, q} \leq \mathfrak{C}(\xi, \sigma, q)\left\|\mathcal{B}_{S}(G)\right\|_{q}$ for $q \in(1,6 / 5]$. The estimate of $\|G\|_{2, q}$ stated in Theorem 8.2 now follows from Lemma 8.4.

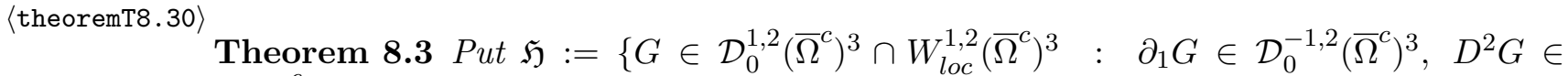
$\left.L^{2}\left(\bar{\Omega}^{c}\right)^{27}, \operatorname{div} G=0\right\}, \mathfrak{A}(G):=\mathcal{P}_{2}\left(-\Delta G+\tau \partial_{1} G\right)$ for $G \in \mathfrak{H}$.

Then $\mathfrak{A}: \mathfrak{H} \mapsto \mathcal{D}_{0}^{-1,2}\left(\bar{\Omega}^{c}\right)^{3} \cap H_{2}\left(\bar{\Omega}^{c}\right)$ is well defined, linear and bijective, with $\mathfrak{A}^{-1}(\phi)=$ $\mathfrak{V}(0, \phi)$ for $\phi \in \mathcal{D}_{0}^{-1,2}\left(\bar{\Omega}^{c}\right)^{3} \cap H_{2}\left(\bar{\Omega}^{c}\right)$.

Proof: Obviously $-\Delta G+\tau \partial_{1} G \in L^{2}\left(\bar{\Omega}^{c}\right)^{3}$ for $G \in \mathfrak{H}$. Moreover, since $\partial_{l} \partial_{m} G_{j}, \partial_{m} G_{j} \in$ $L^{2}\left(\bar{\Omega}^{c}\right)$ for $1 \leq j, l, m \leq 3$, we have $\Delta G \in \mathcal{D}_{0}^{-1,2}\left(\bar{\Omega}^{c}\right)^{3}$ for $G \in \mathfrak{H}$, hence $-\Delta G+\tau \partial_{1} G \in$ $\mathcal{D}_{0}^{-1,2}\left(\bar{\Omega}^{c}\right)^{3}$. At this point Theorem 3.13 implies that $\mathcal{P}_{2}\left(-\Delta G+\tau \partial_{1} G\right) \in \mathcal{D}_{0}^{-1,2}\left(\bar{\Omega}^{c}\right)^{3} \cap$ $H_{2}\left(\bar{\Omega}^{c}\right)$ for $G \in \mathfrak{H}$. Thus $\mathfrak{A}: \mathfrak{H} \mapsto \mathcal{D}_{0}^{-1,2}\left(\bar{\Omega}^{c}\right)^{3} \cap H_{2}\left(\bar{\Omega}^{c}\right)$ is well defined.

Let $\phi \in \mathcal{D}_{0}^{-1,2}\left(\bar{\Omega}^{c}\right)^{3} \cap H_{2}\left(\bar{\Omega}^{c}\right)$. By Theorem 7.3 and Corollary 7.6, we have $\mathfrak{V}(0, \phi) \in \mathfrak{H}$, and there is a function $\Pi(\phi) \in W^{1,2}\left(\bar{\Omega}^{c}\right)$ such that (1.18) holds with $V=\mathfrak{V}(0, \phi), \Pi=\Pi(\phi)$ and $\lambda=0$. Since $\mathcal{P}_{2}(\nabla \Pi(\phi))=0$ (Corollary 3.7), we may conclude from (1.18) that $\mathcal{P}_{2}\left(-\Delta \mathfrak{V}(0, \phi)+\tau \partial_{1} \mathfrak{V}(0, \phi)\right)=\mathcal{P}_{2}(\phi)=\phi$, with the last equation being valid because $\phi \in H_{2}\left(\bar{\Omega}^{c}\right)$. Hence $\mathfrak{A}(\mathfrak{V}(0, \phi))=\phi$. In particular the operator $\mathfrak{A}$ is onto.

Let $G \in \mathfrak{H}$ with $\mathfrak{A}(G)=0$. Then, by the properties of $\mathcal{P}_{2}$ (Theorem 2.1, Corollary 3.7), we get for $\psi \in C_{0}^{\infty}\left(\bar{\Omega}^{c}\right)^{3}$ with $\operatorname{div} \psi=0$ that $\int_{\bar{\Omega}^{c}}\left(\nabla G \cdot \nabla \psi+\tau \partial_{1} G \cdot \psi\right) d x=0$. In view of the regularity properties of $G$ and the equation $\operatorname{div} G=0$, we may now apply Theorem 6.2 , to obtain $G=0$. Therefore $\mathfrak{A}$ is one-to-one. This completes the proof of Theorem 8.3.

From this point onwards, we suppose that problem (2.5) admits only the solution $V=0$ in the space of all functions $V \in \mathcal{D}_{0}^{1,2}\left(\bar{\Omega}^{c}\right)^{3} \cap W_{l o c}^{2,1}\left(\bar{\Omega}^{c}\right)^{3}$ with $D^{2} V \in L^{2}\left(\bar{\Omega}^{c}\right)^{27}$. This is the 
condition required in Theorem 2.5.

$\langle$ theoremT8.40

Theorem 8.4 The relation $\mathcal{P}_{2}(\mathcal{B}(G)) \in \mathcal{D}_{0}^{-1,2}\left(\bar{\Omega}^{c}\right)^{3} \cap H_{2}\left(\bar{\Omega}^{c}\right)$ holds for $G \in \mathfrak{H}$. Define $\widetilde{\mathfrak{A}}(G):=\mathfrak{A}(G)-\mathcal{P}_{2}(\mathcal{B}(G))$ for $G \in \mathfrak{H}$. Then the operator $\widetilde{\mathfrak{A}}: \mathfrak{H} \mapsto \mathcal{D}_{0}^{-1,2}\left(\bar{\Omega}^{c}\right)^{3} \cap H_{2}\left(\bar{\Omega}^{c}\right)$ is well defined, linear and bijective.

Proof: Let $V \in \mathcal{D}_{0}^{1,2}\left(\bar{\Omega}^{c}\right)^{3}$ be a solution to (4.6). We show that $V \in W_{l o c}^{2,1}\left(\bar{\Omega}^{c}\right)^{3}$ and $D^{2} V \in L^{2}\left(\bar{\Omega}^{c}\right)^{27}$. To this end, we observe that $\mathcal{P}_{2}(\mathcal{B}(V)) \in \mathcal{D}_{0}^{-1,2}\left(\bar{\Omega}^{c}\right)^{3} \cap H_{2}\left(\bar{\Omega}^{c}\right)$ by Lemma 4.1, a reference which also yields the first claim of Theorem 8.4. We further observe that by (2.5), the function $V$ satisfies the equation $\operatorname{div} V=0$ as well as (4.1) with $\lambda=0$ and $G=\mathcal{P}_{2}(\mathcal{B}(V))$. On the other hand, by Theorem 7.3 and Corollary 7.4, we know that $\widetilde{V}:=\mathfrak{V}\left(0, \mathcal{P}_{2}[\mathcal{B}(V)]\right) \in \mathcal{D}_{0}^{1,2}\left(\bar{\Omega}^{c}\right)^{3}$, $\operatorname{div}(\widetilde{V})=0$, and $\widetilde{V}$ satisfies (4.1) in the role of $V$, with $\lambda=0$ and $G=\mathcal{P}_{2}(\mathcal{B}(V))$, Now Theorem 6.2 yields $V=\widetilde{V}$, so we may conclude with Corollary 7.6 that $D^{2} V \in L^{2}\left(\bar{\Omega}^{c}\right)^{27}$. Moreover, by (4.6) and because $V \in \mathcal{D}_{0}^{1,2}\left(\bar{\Omega}^{c}\right)^{3}, \mathcal{P}_{2}(\mathcal{B}(V)) \in H_{2}\left(\bar{\Omega}^{c}\right)$, Corollary 4.1 yields a function $\Pi \in W_{\text {loc }}^{1,2}\left(\bar{\Omega}^{c}\right)$ such that the pair $(V, \Pi)$ satisfies (4.5) with $\lambda=0$ and $G=\mathcal{P}_{2}(\mathcal{B}(V))$. Since $D^{2} V \in L^{2}\left(\bar{\Omega}^{c}\right)^{27}$ and $\mathcal{P}_{2}(\mathcal{B}(V)) \in H_{2}\left(\bar{\Omega}^{c}\right)$, the first equation in (4.5) with $\lambda=0$ allows to conclude that $\nabla \Pi \in L^{2}\left(\bar{\Omega}^{c}\right)^{3}$. Thus, due to Theorem 2.1 and Corollary 3.7, we see that (2.5) follows from (4.5) with $\lambda=0$. At this point the condition introduced in the passage preceding Theorem 8.4 implies $V=0$. So the assumptions of Corollary 4.7 are fulfilled, hence we obtain the existence result constituting its conclusion. Now Theorem 8.4 may be proved in the same way as [12, Theorem 17], except that we have to refer to Lemma 4.1, Theorem 8.3 and 6.2 instead of [12, Lemma 8, Theorem 16 and Theorem 7], respectively. The existence assumption being part of $[12,(5.1)]$ is replaced by the conclusion of Corollary 4.7.

corollaryC8.20) Corollary 8.2 The operator $\widetilde{Z}_{0}: \mathcal{D}_{0}^{-1,2}\left(\bar{\Omega}^{c}\right)^{3} \cap H_{2}\left(\bar{\Omega}^{c}\right) \mapsto \mathcal{D}_{0}^{-1,2}\left(\bar{\Omega}^{c}\right)^{3} \cap H_{2}\left(\bar{\Omega}^{c}\right)$ with $\widetilde{Z}_{0}(G):=G-\mathcal{P}_{2}[\mathcal{B}(\mathfrak{V}(0, G))]$ is well defined, linear, bijective and bounded with respect to the norm \|\|$_{*}$

Proof: The bijectivity of $\widetilde{Z}_{0}$ follows from Theorem 8.3 and 8.4, via the abstract - but obvious - operator theoretical equations in [12, Lemma 11]. The boundedness of $\widetilde{Z}_{0}$ is a consequence of the first inequality in Lemma 8.1.

$\langle$ theoremT8.50〉

Theorem 8.5 Let $q \in(1,2)$, and put $Z_{0}^{(q)}(G):=G-\mathcal{P}_{q}[\mathcal{B}(\mathfrak{V}(0, G))]$ for $G \in L^{q}\left(\bar{\Omega}^{c}\right)^{3}$. Then the operator $Z_{0}^{(q)}:=L^{q}\left(\bar{\Omega}^{c}\right)^{3} \mapsto L^{q}\left(\bar{\Omega}^{c}\right)^{3}$ is well defined, linear, bounded and bijective.

Proof: By the second inequality in Lemma 8.1, $Z_{0}^{(q)}$ as an operator from the space $L^{q}\left(\bar{\Omega}^{c}\right)^{3}$ into itself is well defined and bounded. In order to prove that $Z^{(0)}$ is bijective, we consider $R \in(0, \infty)$ with $\bar{\Omega} \subset B_{R}$ and write $Z_{0}^{(q)}$ as a sum $\mathfrak{G}_{R}+\mathfrak{S}_{R}$, with $\mathfrak{G}_{R}(G):=$ $G-\mathcal{P}_{q}\left[\chi_{B_{R}^{c}} \mathcal{B}(\mathfrak{V}(0, G))\right], \mathfrak{S}_{R}(G):=\mathcal{P}_{q}\left[\chi_{B_{R}} \mathcal{B}(\mathfrak{V}(0, G))\right]$ for $G \in L^{q}\left(\bar{\Omega}^{c}\right)^{3}$. Suppose we know that $\mathfrak{S}_{R}$ is compact and $\mathfrak{G}_{R}: L^{q}\left(\bar{\Omega}^{c}\right)^{3} \mapsto L^{q}\left(\bar{\Omega}^{c}\right)^{3}$ is bijective for large $R$. Then the operator $Z_{0}^{(q)}$ is Fredholm with index zero. The proof of bijectivity of $Z_{0}^{(q)}$ is thus reduced to showin that $Z^{(q)}$ is one-to-one. With this in mind, we consider $\phi \in L^{q}\left(\bar{\Omega}^{c}\right)^{3}$ with $Z_{0}^{(q)}(\phi)=0$. Suppose we can deduce from this equation that

$$
\phi \in \mathcal{D}_{0}^{-1,2}\left(\bar{\Omega}^{c}\right)^{3} \cap H_{2}\left(\bar{\Omega}^{c}\right) \cap L^{6 / 5}\left(\bar{\Omega}^{c}\right)^{3} .
$$


In this situation, Corollary 7.4 yields $Z_{0}^{(q)}(\phi)=\widetilde{Z}_{0}(\phi)$, with $\widetilde{Z}_{0}$ from Corollary 8.2. Thus $\widetilde{Z}_{0}(\phi)=0$, so Corollary 8.2 yields $\phi=0$, a conclusion which completes the proof once we have explained why $\mathfrak{S}_{R}$ is compact, $\mathfrak{G}_{R}$ is bijective for large $R$, and (8.22) holds for $\phi \in L^{q}\left(\bar{\Omega}^{c}\right)^{3}$ with $Z_{0}^{(q)}(\phi)=0$. But for all this we refer to [12, proof of Theorem 18], with the results from [12] used in that proof replaced by corresponding results from the work at hand. More precisely, the first two estimates in Theorem 7.1 are substituted for $[12$, (3.16) - (3.18)], whereas assumptions [12, (2.14) - (2.16)] are replaced by (1.5). Moreover the estimate of the term $\left\|D^{2} \mathfrak{V}(\lambda, G) \mid \Omega_{R}\right\|_{q}$ in Theorem 7.1 takes the role of $[12$, Corollary 1], the last inequality in Lemma 8.1 is used instead of [12, (4.4)], Lemma 4.1 fills in for [12, Lemma 8], and Theorem 2.1 and Corollary 3.7 replace [12, Theorem 4]. The function $\mathfrak{V}(0, \phi)$ is used instead of $E^{(0)} * \phi$, for $\phi \in L^{q}\left(\bar{\Omega}^{c}\right)^{3}$. Note that on [12, page 228], it should read (3.16), (3.17) instead of (3.15), (3.16), and the factor $(2 \tau)^{-1}$ in $[12,(5.6)]$ should be replaced by $1 / 2$.

Corollary 8.3 Let $q \in(1,2), \quad \lambda \in K_{\tau} \backslash\{0\}$. Then the operators $\widetilde{Z}_{\lambda}: \mathcal{D}_{0}^{-1,2}\left(\bar{\Omega}^{c}\right)^{3} \cap$ $H_{2}\left(\bar{\Omega}^{c}\right) \mapsto \mathcal{D}_{0}^{-1,2}\left(\bar{\Omega}^{c}\right)^{3} \cap H_{2}\left(\bar{\Omega}^{c}\right), \widetilde{Z}_{\lambda}(\phi):=\phi-\mathcal{P}_{2}[\mathcal{B}(\mathfrak{V}(\lambda, \phi))]$, and $Z_{\lambda}^{(q)}:=L^{q}\left(\bar{\Omega}^{c}\right)^{3} \mapsto$ $L^{q}\left(\bar{\Omega}^{c}\right)^{3}, Z_{\lambda}^{(q)}(\phi):=\phi-\mathcal{P}_{q}[\mathcal{B}(\mathfrak{V}(\lambda, \phi))]$, are well defined, linear and bounded with respect to the norm \|\|$_{*}$ in the case of $\widetilde{Z}_{\lambda}$, and and with respect to \|\|$_{q}$ as concerns $Z_{\lambda}^{(q)}$. If $\psi, G \in \mathcal{D}_{0}^{-1,2}\left(\bar{\Omega}^{c}\right)^{3} \cap H_{2}\left(\bar{\Omega}^{c}\right)$ with $\widetilde{Z}_{\lambda}(\psi)=G$, then $\mathfrak{V}(\lambda, \psi) \in W^{2,2}\left(\bar{\Omega}^{c}\right)^{3}$, and there is $\Gamma \in W_{\text {loc }}^{1,2}\left(\bar{\Omega}^{c}\right)$ with $\nabla \Gamma \in L^{2}\left(\bar{\Omega}^{c}\right)^{3}$ such that (1.19) is satisfied with $V$, П replaced by $\mathfrak{V}(\lambda, \psi)$ and $\Gamma$, respectively. If in addition $G \in L^{q}\left(\bar{\Omega}^{c}\right)^{3}$, then $\psi \in L^{q}\left(\bar{\Omega}^{c}\right)^{3}$, hence $Z_{\lambda}^{(q)}(\psi)=G$.

Proof: It is an immediate consequence of Lemma 8.1 that $\widetilde{Z}_{\lambda}$ and $Z_{\lambda}^{(q)}$ are well defined and bounded. Let $S \in(0, \infty)$ with $\bar{\Omega} \subset B_{S}, G \in \mathcal{D}_{0}^{-1,2}\left(\bar{\Omega}^{c}\right)^{3} \cap H_{2}\left(\bar{\Omega}^{c}\right)$. Then, for $\phi \in$ $\mathcal{D}_{0}^{-1,2}\left(\bar{\Omega}^{c}\right)^{3}$, we have $\mathcal{B}(\mathfrak{V}(\lambda, \phi)), \mathcal{P}_{2}[\mathcal{B}(\mathfrak{V}(\lambda, \phi))] \in L^{p}\left(\bar{\Omega}^{c}\right)^{3}$ for $p \in(1,2]$ according to Lemma 8.1, so Corollary 7.1 allows us to consider

$$
V_{\phi}:=\mathfrak{V}\left(\lambda, \mathcal{P}_{2}[\mathcal{B}(\mathfrak{V}(\lambda, \phi))]+G\right), \quad \Pi_{\phi}:=\Pi\left(\lambda, \mathcal{P}_{2}[\mathcal{B}(\mathfrak{V}(\lambda, \phi))]+G\right) .
$$

This corollary implies that $V_{\phi} \in W^{2,2}\left(\bar{\Omega}^{c}\right)^{3}, \Pi_{\phi} \in W_{l o c}^{1,2}\left(\bar{\Omega}^{c}\right), \nabla \Pi_{\phi} \in L^{2}\left(\bar{\Omega}^{c}\right)^{3}$, and that the pair $\left(V_{\phi}, \Pi_{\phi}\right)$ solves (1.18) with $G$ replaced by $\mathcal{P}_{2}[\mathcal{B}(\mathfrak{V}(\lambda, \phi))]+G$, where $\phi$ may be any function from $\mathcal{D}_{0}^{-1,2}\left(\bar{\Omega}^{c}\right)^{3}$. Now suppose there is $\psi \in \mathcal{D}_{0}^{-1,2}\left(\bar{\Omega}^{c}\right)^{3} \cap H_{2}\left(\bar{\Omega}^{c}\right)$ with $\widetilde{Z}_{\lambda}(\psi)=G$. Then we get $\mathfrak{V}(\lambda, \psi)=V_{\psi}$. Putting $\Gamma:=\Pi_{\psi}+\mathcal{G}_{2}[\mathcal{B}(\mathfrak{V}(\lambda, \psi))]$, with $\mathcal{G}_{2}$ from Theorem 2.1 , we may conclude that $\Gamma \in W_{l o c}^{1,2}\left(\bar{\Omega}^{c}\right), \nabla \Gamma \in L^{2}\left(\bar{\Omega}^{c}\right)^{3}$, and that the pair $(\mathfrak{V}(\lambda, \psi), \Gamma)$ solves (1.19). Suppose in addition that $G \in L^{q}\left(\bar{\Omega}^{c}\right)^{3}$. Since $\mathcal{P}_{2}[\mathcal{B}(\mathfrak{V}(\lambda, \psi))] \in L^{p}\left(\bar{\Omega}^{c}\right)^{3}$ for any $p \in(1,2]$, as mentioned above, we then obtain $\psi \in L^{q}\left(\bar{\Omega}^{c}\right)^{3}$.

Theorem 8.6 There is $\epsilon_{1} \in\left(0,(\tau / 2)^{2}\right]$ such that for any $\lambda \in \mathbb{C} \backslash\{0\}$ with $\Re \lambda \geq 0,|\lambda| \leq$ $\epsilon_{1}$, the operator $\widetilde{Z}_{\lambda}: \mathcal{D}_{0}^{-1,2}\left(\bar{\Omega}^{c}\right)^{3} \cap H_{2}\left(\bar{\Omega}^{c}\right) \mapsto \mathcal{D}_{0}^{-1,2}\left(\bar{\Omega}^{c}\right)^{3} \cap H_{2}\left(\bar{\Omega}^{c}\right)$ is bijective, with $\|\phi\|_{*} \leq$ $\mathfrak{C}\left\|\widetilde{Z}_{\lambda}(\phi)\right\|_{*}$ for $\phi \in \mathcal{D}_{0}^{-1,2}\left(\bar{\Omega}^{c}\right)^{3} \cap H_{2}\left(\bar{\Omega}^{c}\right)$.

Let $q \in(1,2)$. Then there is $\epsilon_{2}(q) \in\left(0,(\tau / 2)^{2}\right]$ such that for $\lambda \in \mathbb{C} \backslash\{0\}$ with $\Re \lambda \geq$ $0,|\lambda| \leq \epsilon_{2}(q)$, the operator $Z_{\lambda}^{(q)}:=L^{q}\left(\bar{\Omega}^{c}\right)^{3} \mapsto L^{q}\left(\bar{\Omega}^{c}\right)^{3}$ is bijective, and such that the estimate $\|G\|_{q} \leq \mathfrak{C}(q)\left\|Z_{\lambda}^{(q)}(G)\right\|_{q}$ holds for $G \in L^{q}\left(\bar{\Omega}^{c}\right)^{3}$.

Proof: This theorem follows from Theorem 8.1 - via Corollary 8.1 - and Lemma 8.1. The 
reasoning starts with Corollary 8.2 and Theorem 8.5, which provide constants $\widetilde{C}_{0}, C_{0}(q)>$ 0 such that

$$
\|\phi\|_{*} \leq \widetilde{C}_{0}\left\|\widetilde{Z}_{\lambda}(\phi)\right\|_{*}, \quad\|G\|_{q} \leq C_{0}(q)\left\|Z_{\lambda}^{(q)}(G)\right\|_{q}
$$

for $\phi, G$ as in the theorem. Take such a function $\phi$. By Lemma 8.1 and Corollary 8.1, we find that

$$
\begin{aligned}
& \left\|\widetilde{Z}_{\lambda}(\phi)-\widetilde{Z}_{0}(\phi)\right\|_{*}=\left\|\mathcal{P}_{2}[\mathcal{B}(\mathfrak{V}(\lambda, \phi)-\mathfrak{V}(0, \phi))]\right\|_{*} \\
& \leq\left\|\mathcal{P}_{2}\left[\chi_{B_{R}^{c}} \mathcal{B}(\mathfrak{V}(\lambda, \phi))\right]\right\|_{*}+\left\|\mathcal{P}_{2}\left[\chi_{B_{R}^{c}} \mathcal{B}(\mathfrak{V}(0, \phi))\right]\right\|_{*} \\
& \quad+\left\|\mathcal{P}_{2}\left[\chi_{B_{R}} \mathcal{B}(\mathfrak{V}(\lambda, \phi)-\mathfrak{V}(0, \phi))\right]\right\|_{*} \\
& \leq\left(2 D_{1}(R)+C_{1}(R)\left[\widetilde{R}^{-1 / 2}+(-\ln (1-1 / \widetilde{R}))^{1 / 2}\right]+C_{2}(R, \widetilde{R})|\lambda|^{1 / 3}\right)\|\phi\|_{*}
\end{aligned}
$$

for $R \in(0, \infty)$ with $\bar{\Omega} \subset B_{R}, \widetilde{R} \in[2 R+3, \infty), \lambda \in K_{\tau} \backslash\{0\}$, where $D_{1}(R)$ was introduced in Lemma 8.1. The specific (not generic) constants $C_{1}(R)$ and $C_{2}(R, \widetilde{R})$ introduced here do not depend on either $\lambda$ or $\phi$. Since $D_{1}(R) \rightarrow 0$ for $R \rightarrow \infty$, we may choose $R>0$ with $\bar{\Omega} \subset B_{R}$ and $2 D(R) \leq\left(12 \widetilde{C}_{0}\right)^{-1}$, with $\widetilde{C}_{0}$ from $(8.23)$. Next we fix $\widetilde{R} \in[2 R+3, \infty)$ such that $C_{1}(R)\left[\widetilde{R}^{-1 / 2}+(-\ln (1-1 / \widetilde{R}))^{1 / 2}\right] \leq\left(12 \widetilde{C}_{0}\right)^{-1}$, and finally we choose $\epsilon_{1} \in$ $\left(0,(\tau / 2)^{2}\right]$ so small that $C_{2}(R, \widetilde{R}) \epsilon_{1}^{1 / 3} \leq\left(12 \widetilde{C}_{0}\right)^{-1}$. Then, if $\lambda \in K_{\tau} \backslash\{0\}$ with $|\lambda| \leq \epsilon_{1}$, we may deduce from (8.23) and $(8.24)$ that $\left\|\widetilde{Z}_{\lambda}(\phi)\right\|_{*} \geq\left\|\widetilde{Z}_{0}(\phi)\right\|_{*}-\left\|\widetilde{Z}_{\lambda}(\phi)-\widetilde{Z}_{0}(\phi)\right\|_{*} \geq$ $\widetilde{C}_{0}^{-1}\|\phi\|_{*}-\left(2 \widetilde{C}_{0}\right)^{-1}\|\phi\|_{*}=\left(2 \widetilde{C}_{0}\right)^{-1}\|\phi\|_{*}$. Applying Lemma 8.1 and Corollary 8.1 in an analogous way, we may find $\epsilon_{2}(q) \in\left(0,(\tau / 2)^{2}\right]$ with $\left\|Z_{\lambda}^{(q)}(G)\right\|_{q} \geq\left(2 C_{0}(q)\right)^{-1}\|G\|_{q}$ for $G$ as in the theorem and for $\lambda \in \mathbb{C} \backslash\{0\}$ with $\Re \lambda \geq 0,|\lambda| \leq \epsilon_{2}(q)$, where $C_{0}(q)$ was introduced in (8.23). As for the bijectivity of $\widetilde{Z}_{\lambda}$ and $Z_{\lambda}^{(q)}$, we refer to the last part of the proof of [12, Theorem 19].

Now we consider the operator $\mathcal{L}$ defined in $(1.14)$, with a domain $\mathcal{D}(\mathcal{L})$ as chosen in $(1.11)$. Recall that the identical mapping of $H_{2}\left(\bar{\Omega}^{c}\right)$ onto itself is denoted by $\mathfrak{I}$.

Lemma 8.5 The relation $-\Delta V+\tau \partial_{1} V-\mathcal{B}(V) \in L^{2}\left(\bar{\Omega}^{c}\right)^{3}$ holds for $V \in \mathcal{D}(\mathcal{L})$, hence $\mathcal{L}$ is well defined. The set $\mathcal{D}(\mathcal{L})$ is dense in $H_{2}\left(\bar{\Omega}^{c}\right)$, and $\mathcal{L}: \mathcal{D}(\mathcal{L}) \mapsto H_{2}\left(\bar{\Omega}^{c}\right)$ is linear and closed as an operator from a subspace of $H_{2}\left(\bar{\Omega}^{c}\right)$ into $H_{2}\left(\bar{\Omega}^{c}\right)$.

Proof: Since $\mathcal{D}(\mathcal{L}) \subset \mathcal{D}_{0}^{1,2}\left(\bar{\Omega}^{c}\right)^{3}$, Lemma 4.1 yields the first statement of Lemma 8.5. Let $V \in \mathcal{D}(\mathcal{L})$, and put $\Pi:=\mathcal{G}_{2}\left(\Delta V-\tau \partial_{1} V+\mathcal{B}(V)\right)$. Theorem 2.1 implies that $\Pi \in$ $W_{\text {loc }}^{1,2}\left(\bar{\Omega}^{c}\right), \nabla \Pi \in L^{2}\left(\bar{\Omega}^{c}\right)^{3}$, and the pair $(V, \Pi)$ solves $(1.19)$ with $G=-\mathcal{L}(V)+V$ and $\lambda=1$. Therefore $\|V\|_{2,2} \leq\|-\mathcal{L}(V)+V\|_{2}$ by Theorem 4.9. From this it may be deduced that $\mathcal{L}$ is closed.

We will make use of the following result about eigenvalues of $\mathcal{L}$. Recall that $\varrho(\mathcal{L})$ denotes the resolvent set of $\mathcal{L}$.

Theorem 8.7 ([2], [17]) The set $\mathfrak{K}:=\varrho(\mathcal{L}) \backslash\left\{\lambda \in \mathbb{C}: \tau^{2} \Re \lambda \leq-(\Im \lambda)^{2}\right\}$ is at most countable and consists of eigenvalues of $\mathcal{L}$.

From now on we require that $\Re \lambda<0$ for any $\lambda \in \mathfrak{K}$. In view of the assumptions on the unicity of solutions to problem (2.5), imposed in the passage preceding Theorem 8.4, we 
have thus introduced all the assumptions required in Theorem 2.5. In particular we have

$$
\{\lambda \in \mathbb{C}: \Re \lambda \geq 0, \lambda \neq 0\} \subset \varrho(\mathcal{L}) .
$$

Corollary 8.4 Let $\lambda \in \mathbb{C} \backslash\{0\}$ with $\Re \lambda \geq 0,|\lambda| \leq \epsilon_{1}$, where $\epsilon_{1}$ was introduced in Theorem 8.6. Let $G \in \mathcal{D}_{0}^{-1,2}\left(\bar{\Omega}^{c}\right)^{3} \cap H_{2}\left(\bar{\Omega}^{c}\right)$. Then $(\lambda \mathfrak{I}-\mathcal{L})^{-1}(G)=\mathfrak{V}\left(\lambda,\left(\widetilde{Z}_{\lambda}\right)^{-1}(G)\right)$. There is $\Gamma \in W_{\text {loc }}^{1,2}\left(\bar{\Omega}^{c}\right)$ such that $\nabla \Pi \in L^{2}\left(\bar{\Omega}^{c}\right)^{3}$ and (1.19) is satisfied with $V$, $\Pi$ replaced by $(\lambda \mathfrak{I}-\mathcal{L})^{-1}(G)$ and $\Gamma$, respectively. Moreover $(\lambda \mathfrak{I}-\mathcal{L})^{-1}(G) \in \mathcal{D}_{0}^{-1,2}\left(\bar{\Omega}^{c}\right)^{3}$.

Proof: Abbreviate $W:=\mathfrak{V}\left(\lambda,\left(\widetilde{Z}_{\lambda}\right)^{-1}(G)\right)$. We have $\left(\widetilde{Z}_{\lambda}\right)^{-1}(G) \in \mathcal{D}_{0}^{-1,2}\left(\bar{\Omega}^{c}\right)^{3}$ (Theorem 8.6), so $W \in \mathcal{D}_{0}^{1,2}\left(\bar{\Omega}^{c}\right)^{3}$ (Theorem 7.3, Corollary 7.4). Moreover Corollary 8.3 yields $W \in$ $W^{2,2}\left(\bar{\Omega}^{c}\right)^{3}$ and existence of a function $\Gamma \in W_{l o c}^{1,2}\left(\bar{\Omega}^{c}\right)$ with $\nabla \Gamma \in L^{2}\left(\bar{\Omega}^{c}\right)^{3}$ and satisfying (1.19) with $V, \Pi$ replaced by $W, \Gamma$, respectively. On the other hand, $\lambda \in \varrho(\mathcal{L})$ by $(8.25)$, so there is $\widetilde{W} \in \mathcal{D}(\mathcal{L})$ with $(\lambda \mathfrak{I}-\mathcal{L})(\widetilde{W})=G$. Putting $\widetilde{\Gamma}:=\mathcal{G}_{2}\left(-\Delta \widetilde{W}+\tau \partial_{1} \widetilde{W}-\mathcal{B}(\widetilde{W})\right)$ (see the first statement in Lemma 8.1), we get with Theorem 2.1 that (1.19) holds with $\widetilde{W}, \widetilde{\Gamma}$ in the role of $V$ and $\Pi$, respectively. Now Theorem 6.2 yields $W=\widetilde{W}$, that is, $W=(\lambda \mathfrak{I}-\mathcal{L})^{-1}(G)$. We have $\widetilde{W} \in \mathcal{D}(\mathcal{L}) \subset \mathcal{D}_{0}^{1,2}\left(\bar{\Omega}^{c}\right)^{3}$, so $\mathcal{P}_{2}(\mathcal{B}(\widetilde{W})) \subset \mathcal{D}_{0}^{-1,2}\left(\bar{\Omega}^{c}\right)^{3}$ by Lemma 4.1. Moreover, since $\widetilde{W} \in \mathcal{D}(\mathcal{L}) \subset W^{2,2}\left(\bar{\Omega}^{c}\right)^{3} \cap H_{2}\left(\bar{\Omega}^{c}\right)$, we further have $\partial_{1} \widetilde{W} \in \mathcal{D}_{0}^{-1,2}\left(\bar{\Omega}^{c}\right)^{3}, D^{2} \widetilde{W} \in L^{2}(\bar{\Omega})^{27}$ and $\operatorname{div} \widetilde{W}=0$ (Lemma 3.2). Thus, referring to Theorem 8.3, we get $\widetilde{W} \in \mathfrak{H}$ and $\mathfrak{A}(\widetilde{W}) \in \mathcal{D}_{0}^{-1,2}\left(\bar{\Omega}^{c}\right)^{3}$. But $(\lambda \mathfrak{I}-\mathcal{L})(\widetilde{W})=G$ by the choice of $\widetilde{W}$, so $\widetilde{W}=\lambda^{-1}(\mathcal{L}(\widetilde{W})+G)=\lambda^{-1}\left[-\mathfrak{A}(\widetilde{W})+\mathcal{P}_{2}(\mathcal{B}(\widetilde{W})+G]\right.$. Thus we have found that $\widetilde{W} \in \mathcal{D}_{0}^{-1,2}\left(\bar{\Omega}^{c}\right)^{3}$. This proves the last claim of Corollary 8.4.

The ensuing theorem states our resolvent estimates for solutions to the perturbed Oseen system in (1.19), under the assumption that the resolvent parameter is small.

$\langle$ theoremT8.80〉

Theorem 8.8 The inequality $\left\|\nabla(\lambda \mathfrak{I}-\mathcal{L})^{-1}(G)\right\|_{2} \leq \mathfrak{C}\|G\|_{*}$ holds for $G \in \mathcal{D}_{0}^{-1,2}\left(\bar{\Omega}^{c}\right)^{3} \cap$ $H_{2}\left(\bar{\Omega}^{c}\right), \lambda \in \mathbb{C} \backslash\{0\}$ with $\Re \lambda \geq 0,|\lambda| \leq \epsilon_{1}$, where $\epsilon_{1}$ was introduced in Theorem 8.6.

Let $s \in(1,6 / 5], \delta \in(0,1]$. Then there is $\epsilon_{3}(s, \delta) \in\left(0, \epsilon_{1}\right]$ such that for $G \in L^{s}\left(\bar{\Omega}^{c}\right)^{3} \cap$ $H_{2}\left(\bar{\Omega}^{c}\right), R \in(0, \infty)$ with $\bar{\Omega} \subset B_{R}, \lambda \in \mathbb{C} \backslash\{0\}$ with $\Re \lambda \geq 0,|\lambda| \leq \epsilon_{3}(s, \delta)$, the ensuing estimates hold:

$$
\begin{aligned}
& \left\|\nabla(\lambda \mathfrak{I}-\mathcal{L})^{-2}(G)\left|\Omega_{R}\left\|_{2}+\right\| \nabla\left((\bar{\lambda} \mathfrak{I}-\mathcal{L})^{-1} \circ(\lambda \mathfrak{I}-\mathcal{L})^{-1}(G)\right)\right| \Omega_{R}\right\|_{2} \\
& \leq \mathfrak{C}(s, \delta, R)|\lambda|^{-4(1-1 / s))-\delta}\|G\|_{s}, \\
& \left\|\nabla ( \lambda \mathfrak { I } - \mathcal { L } ) ^ { - 3 } ( G ) \left|\Omega_{R}\left\|_{2} \leq \mathfrak{C}(s, \delta, R)|\lambda|^{-6+4 / s-\delta}\right\| G \|_{s} .\right.\right.
\end{aligned}
$$

Proof: The first part of this theorem is an immediate consequence of the equation $(\lambda \mathfrak{I}-$ $\mathcal{L})^{-1}(G)=\mathfrak{V}\left(\lambda,\left(\widetilde{Z}_{\lambda}\right)^{-1}(G)\right)$ (Corollary 8.4), Theorem 7.3 and Theorem 8.6; see the beginning of the proof of [12, Theorem 21]. As concerns the other inequalities stated in the theorem, they are proved in exactly the same way as the corresponding estimates in [12, Theorem 21], except that the references to [12, Lemma 12, Theorem 19, Corollary 4, (3.15), (3.17)] are to be replaced by Corollary 8.4, Theorem 8.6, Corollary 8.3, and the third and second inequality in Theorem 7.1, respectively. The function $\mathfrak{V}(\lambda, \phi)$ has to be substituted for $E^{(\lambda)} * \phi\left(\phi \in \mathcal{D}_{0}^{-1,2}\left(\bar{\Omega}^{c}\right)^{3} \cap H_{2}\left(\bar{\Omega}^{c}\right)\right)$. Note that a factor $1 / 2$ is lacking in the definition of $p$ on [12, page 231], and the symbol $\nabla$ and the restriction to $B_{R}$ are missing on the left-hand side of the last estimate on [12, page 231], 
Corollary 8.5 The inequality $\left\|\nabla(\lambda \mathfrak{I}-\mathcal{L})^{-1}(G)\right\|_{2} \leq \mathfrak{C}(\xi, \sigma)\|G\|_{*}$ holds for $\xi, \sigma, G$ as in Theorem 8.2 and for $\lambda \in \mathbb{C} \backslash\{0\}$ with $\Re \lambda \geq 0$ and $|\lambda| \leq \epsilon_{1}$.

Let $\delta \in(0,1]$. Then there is $\epsilon_{4}(\delta) \in\left(0, \epsilon_{1}\right]$ such that

$$
\begin{aligned}
& \left\|\nabla(\lambda \mathfrak{I}-\mathcal{L})^{-2}(G)\left|\Omega_{R}\left\|_{2}+\right\| \nabla\left((\bar{\lambda} \mathfrak{I}-\mathcal{L})^{-1} \circ(\lambda \mathfrak{I}-\mathcal{L})^{-1}(G)\right)\right| \Omega_{R}\right\|_{2} \\
& \leq \mathfrak{C}(\xi, \sigma, \delta, R)|\lambda|^{-\delta}\|G\|_{2}, \\
& \left\|\nabla ( \lambda \mathfrak { I } - \mathcal { L } ) ^ { - 3 } ( G ) \left|\Omega_{R}\left\|_{2} \leq \mathfrak{C}(\xi, \sigma, \delta, R)|\lambda|^{-2-\delta}\right\| G \|_{2}\right.\right.
\end{aligned}
$$

for $R \in(0, \infty)$ with $\bar{\Omega} \subset B_{R}, \lambda \in \mathbb{C} \backslash\{0\}$ with $\Re \lambda \geq 0,|\lambda| \leq \epsilon_{4}(\delta)$, and for $\xi, \sigma, G$ as in Theorem 8.2.

Proof: See the proof of [12, Corollary 5]. Use Theorem 8.2 instead of [12, (4.26)], and Theorem 8.8 in the place of $[12$, Theorem 21, (6.3), (6.4)].

Lemma 8.6 Let $\lambda \in \mathbb{C} \backslash\{0\}$ with $\Re \lambda \geq 0$ and $G \in H_{2}\left(\bar{\Omega}^{c}\right)$. Then

$$
\left\|\nabla(\lambda \mathfrak{I}-\mathcal{L})^{-1}(G)\right\|_{2} \leq \mathfrak{C}\left(\|G\|_{2}+\left\|(\lambda \mathfrak{I}-\mathcal{L})^{-1}(G)\right\|_{2}\right) .
$$

Proof: Put $V:=(\lambda \mathfrak{I}-\mathcal{L})^{-1}(G)$. Then, by the definition of $\mathcal{L}$, we have $V \in \mathcal{D}(\mathcal{L})$. By Corollary 8.4, there is $\Pi \in W_{l o c}^{1,2}\left(\bar{\Omega}^{c}\right)$ with $\nabla \Pi \in L^{2}\left(\bar{\Omega}^{c}\right)^{3}$ such that (1.19) holds. Lemma 8.6 follows from this latter equation and from Lemma 4.1, via a variational argument as in the proof of [12, Lemma 13], similar to the end of the proof of Theorem 6.2. The argument in question involves an integration by parts in the integrals $\int_{\bar{\Omega}^{c}}-\Delta u \cdot \bar{u} d x$ and $\int_{\bar{\Omega}^{c}} \partial_{1} u \cdot \bar{u} d x$, with $u \in \mathcal{D}(\mathcal{L})$. These integrations do not generate boundary terms because $\mathcal{D}(\mathcal{L}) \subset W_{0}^{1,2}\left(\bar{\Omega}^{c}\right)^{3}$. Note that the condition $\lambda \neq 0$ was forgotten in [12, Lemma 13].

Lemma 8.7 Let $\gamma_{1}, \gamma_{2} \in(0, \infty)$ with $\gamma_{1}<\gamma_{2}$. Put $\mathfrak{M}_{\gamma_{1}, \gamma_{2}}:=\left\{\lambda \in \mathbb{C}: \Re \lambda \geq 0, \gamma_{1} \leq\right.$ $\left.|\lambda| \leq \gamma_{2}\right\}$. Then $\mathfrak{M}_{\gamma_{1}, \gamma_{2}} \subset \varrho(\mathcal{L})$ and $\left\|(\lambda \mathfrak{I}-\mathcal{L})^{-1}(G)\right\|_{2} \leq \mathfrak{C}\left(\gamma_{1}, \gamma_{2}\right)\|G\|_{2}$ for $G \in H_{2}\left(\bar{\Omega}^{c}\right)$.

Proof: The lemma follows from (8.25); see the proof of [12, Lemma 14]. Use Lemma 8.6 instead of [12, Lemma 13].

We recall that $S_{\vartheta, a}$ stands for the sector $\{\lambda \in \mathbb{C}:|\arg (\lambda-a)| \leq \vartheta\}$ of the complex plane.

Theorem 8.9 Fix some $\vartheta \in(\pi / 2, \pi)$ and some $a_{0} \in(0, \infty)$. Then there is a constant $C_{1}>0$ such that the following two estimates hold: Firstly, $|\lambda|\|V\|_{2} \leq \mathfrak{C}\|G\|_{2}$ for $\lambda \in \mathbb{C}$ with $|\lambda| \geq C_{1}$ and which additionally satisfies at least one of the relations $\Re \lambda \geq 0$ and $\lambda \in S_{\vartheta, a}, G \in H_{2}\left(\bar{\Omega}^{c}\right), V \in \mathcal{D}(\mathcal{L})$ with $(\lambda V-\mathcal{L})(V)=G$. Secondly, $|\lambda|\|\nabla V\|_{2} \leq \mathfrak{C}\|\nabla G\|_{2}$ for $\lambda$ as above, $G \in H_{2}\left(\bar{\Omega}^{c}\right) \cap W_{0}^{1,2}\left(\bar{\Omega}^{2}\right)^{3}$ and $V$ as above.

Proof: A proof of the second estimate in Theorem 8.9 is elaborated in [12, page 233-224] for the whole space case. This proof carries over to the exterior domain case with some minor adaptions. In fact, the equation $\lambda V-\mathcal{L}(V)=G$ has to be multiplied by $\mathcal{P}_{2}(-\Delta \bar{V})$ instead of $-\Delta \bar{V}$. Theorem 2.1 may be used to handle the operator $\mathcal{P}_{2}$. The reference to [12, Lemma 8] may be replaced by one to Lemma 4.1. Note that the integration by parts in the integral $\int_{\bar{\Omega}^{c}} G \cdot \mathcal{P}_{2}(\Delta \bar{V}) d x=\int_{\bar{\Omega}^{c}} G \cdot \Delta \bar{V} d x$ arising in the proof of $[12,(6.15)]$ 
does not generate any boundary terms because we supposed $G \in W_{0}^{1,2}\left(\bar{\Omega}^{c}\right)^{3}$. The first inequality in Theorem 8.9 follows by a variational argument similar to but simpler than the one leading to the second estimate; compare the end of the proof of Theorem 6.2 and [12, proof of Lemma 13] (which corresponds to Lemma 8.6 here) for the case $\Re \lambda \geq 0$.

Corollary 8.6 Fix some $a \in\left[a_{0}, \infty\right)$ so large that $\left\{\sigma \in \mathbb{C}:|\sigma|<C_{1}\right\} \cup\left\{\sigma \in \mathbb{C}: \tau^{2} \Re \sigma \leq\right.$ $\left.(\Im \sigma)^{2}\right\} \subset \mathbb{C} \backslash S_{\vartheta, a}$, with $\vartheta$ and $a_{0}$ from Theorem 8.9. Then

$$
\left\{\lambda \in S_{\vartheta, a}: \Re \lambda<0\right\} \cup\{\lambda \in \mathbb{C} \backslash\{0\}: \Re \lambda \geq 0\} \subset \varrho(\mathcal{L}) .
$$

Moreover

$$
|\lambda|\left\|(\lambda V-\mathcal{L})^{-1}(G)\right\|_{2} \leq \mathfrak{C}\|G\|_{2},
$$

for $G \in H_{2}\left(\bar{\Omega}^{c}\right)$, for $\lambda \in \mathbb{C}$ with $|\lambda| \geq C_{1}$ and $\Re \lambda \geq 0$, and for any $\lambda \in S_{\vartheta, a}$;

$$
|\lambda|\left\|\nabla(\lambda V-\mathcal{L})^{-1}(G)\right\|_{2} \leq \mathfrak{C}\|\nabla G\|_{2}
$$

for $G \in H_{2}\left(\bar{\Omega}^{c}\right) \cap W_{0}^{1,2}\left(\bar{\Omega}^{2}\right)^{3}$ and for $\lambda$ as in (8.27).

Proof: Let $\lambda \in \mathbb{C} \backslash\{0\}$ with $\Re \lambda \geq 0$ or with $\lambda \in S_{\vartheta, a}$ and $\Re \lambda<0$. If $\Re \lambda \geq 0$, we have $\lambda \in \varrho(\mathcal{L})$ by (8.25). Otherwise, if $\lambda \in S_{\vartheta, a}$ and $\Re \lambda<0$, we have $\tau^{2} \Re \sigma>(\Im \sigma)^{2}$ by the choice of $a$, so either $\lambda \in \varrho(\mathcal{L})$ or $\lambda$ is an eigenvalue of $\mathcal{L}$; see Theorem 8.7. But we have $S_{\vartheta, a} \subset\left\{\sigma \in \mathbb{C}:|\sigma| \geq C_{1}\right\}$ again by the choice of $a$. Moreover $S_{\vartheta, a} \subset S_{\vartheta, a_{0}}$ because $a \geq a_{0}$ and $\vartheta>\pi / 2$. Therefore $|\lambda| \geq C_{1}$ and $\lambda \in S_{\vartheta, a_{0}}$, so the eigenvalue case is excluded by the first estimate in Theorem 8.9. As a consequence $\lambda \in \varrho(\mathcal{L})$, and $(8.26)$ is proved. Since $S_{\vartheta, a} \subset S_{\vartheta, a_{0}} \cap\left\{\sigma \in \mathbb{C}:|\sigma| \geq C_{1}\right\}$, inequalities (8.27) and (8.28) follow from Theorem 8.9 and from (8.26).

As mentioned in [12, page 234] in a similar situation, Theorem 8.8 presents resolvent estimates for the operator $\mathcal{L}$ in the case that $|\lambda|$ is small, Lemma 8.7 may be interpreted as dealing with intermediate values of $|\lambda|$, and Corollary 8.6 deals with large values.

\section{Proof of Theorem 2.5.}

Theorem 2.5 may be proved in almost exactly the same way as [12, Theorem 25] is shown in $[12$, section 7]. The reasoning in [12, section 7] is completely functional analytic, building on the preceding results in [12]. So we just have to modifiy the references given in [12] for those preceding results. In this respect we mention that $[12,(6.2)]$ is replaced by (8.26). As concerns [12, Corollary 5], in particular [12, (6.10) - (6.12)], we now make use of Corollary 8.5. We substitute (8.28) for [12, (6.15)], and Theorem 8.2 for [12, Theorem 15], in particular for $[12,(4.26)]$. Instead of [12, Lemma 14], we apply Lemma 8.7. Concerning [12, Theorem 20], it is referred to on [12, page 235] because of the relation $S_{\vartheta, a} \subset \varrho(\mathcal{L})$, for which our reference here is (8.26). As for the parameters $C_{1}, \vartheta, a$ and $\epsilon_{4}(\delta)$ appearing in [12, section 7], we indicate that $C_{1}$ and $\vartheta$ are introduced in Theorem 8.9, whereas we refer to Corollary 8.6 for $a$ and to Corollary 8.5 for $\epsilon_{1}(\delta)$. We recall that by the choice of $a$, we have $|\lambda| \geq C_{1}$ for any $\lambda \in S_{\vartheta, a}$ (Corollary 8.6), a relation that need not be valid in the setup in [12]. But this additional feature of the choice of $a$ here enables us to obtain 
inequality (8.27) for any $\lambda \in S_{\vartheta, a}$. Therefore, according to [26], this inequality implies directly that $\mathcal{L}$ generates an analytical semigroup; see Theorem 2.2 and its proof. In [12], it was implicitly assumed that the constraint $|\lambda| \geq C_{1}$ first has to be removed by shifting the sector $S_{\vartheta, a}$ in the direction of the positive real axis in order to be in accordance with the framework in [26]; see [12, page 235 at the top]. We finally remark that the condition $G \in W_{0}^{1,2}\left(\bar{\Omega}^{c}\right)^{3}$ in (8.28), instead of $G \in W^{1,2}\left(\bar{\Omega}^{c}\right)^{3}$, does not cause any trouble because this inequality is needed only for the case that $G$ is given as in Theorem 8.2, that is, satisfying the relation $\mathcal{D}(\mathcal{L})$. In particular, in a version of [12, Theorem 24] adapted to our situation, we may require that $\phi \in H_{2}\left(\bar{\Omega}^{c}\right) \cap W_{0}^{1,2}\left(\bar{\Omega}^{c}\right)^{3}$; see the proof of $[12$, Theorem $25]$ on [12, page 240 below], where [12, Theorem 24] is applied. The "differential equation stated at the end of Theorem 25" mentioned on [12, page 240 below] means the equation $\Delta f+\xi \mathcal{P} \mathfrak{B}_{\text {sym }} f=\sigma f$, which is only alluded to, but not actually stated, at the end of $[12$, Theorem 25].

\section{References}

aR [1] C. Amrouche and U. Razafison, On the Oseen problem in three-dimensional exterior domains, Anal. Appl. 4 (2006), 133-162.

$\mathrm{Bab}$ [2] K. I. BABEnKo, Spectrum of the linearized problem of flow of a viscous incompressible liquid round a body, Sov. Phys. Dokl. 27 (1982), 25-27.

BM1 [3] W. Borchers And T. MiYakawa, $L^{2}$-decay for Navier-Stokes flows in unbounded domains, with application to exterior stationary flows, Arch. Rat. Mech. Anal. 118 (1992), 273-295.

BM2 [4] W. Borchers and T. MiYaKaWA, On stability of exterior stationary Navier-Stokes flows, Acta Math. 174 (1995), 311-382.

BoSo2 [5] W. Borchers AND H. Sohr, On the semigroup of the Stokes operator for exterior domains in $L^{q}$-spaces, Math. Z. 196 (1987), 415-425.

BoSo [6] W. Borchers And H. Sohr, On the equations $\operatorname{rot} v=g$ and $\operatorname{div} v=f$ with zero boundary conditions, Hokkaido Math. J. 19 (1990), 67-87.

De1R [7] P. Deuring, An integral operator related to the Stokes system in exterior domains, Math. Methods Appl. Sci. 13 (1990), 323-333. Addendum, Math. Methods Appl. Sci. 14 (1991), 445.

De2 [8] P. Deuring, The resolvent problem for the Stokes system in exterior domains: an elementary approach, Math. Meth. in the Appl. Sci. 13 (1990), 335-349.

?DeBirk? [9] P. Deuring, Resolvent estimates for a perturbed Oseen problem. In Functional Analysis and Evolution Equations. The Günter Lumer Volume, H. Amann e.a. (eds.), Birkhäuser, Basel 2007, 171-186.

D-DCDSA [10] P. DeuRING, Pointwise spatial decay of time-dependent Oseen flows: the case of data with noncompact support. Discr. Cont. Dyn. Syst. Ser. A 33 (2013), 2757-2776. 
D-JMFM [11] P. Deuring, Pointwise spatial decay of weak solutions to the Navier-Stokes system in 3D exterior domains. J. Math. Fluid Mech. 17 (2015), 199-232.

DN [12] P. Deuring And J. Neustupa, An eigenvalue criterion for stability of a steady Navier-Stokes flow in $\mathbb{R}^{3}$, J. Math. Fluid Mech. 12 (2010), 202-240.

DV [13] P. Deuring and W. Varnhorn, On Oseen resolvent estimates, Diff. Int. Equat. 23 (2010), 1139-1149.

D-ZAmp [14] P. Deuring, S. KraČmar and Š. NeČasová, Asymptotic structure of viscous incompressible flow around a rotating body, with nonvanishing flow field at infinity. To appear in Z. Angew. Math. Phys. 68 (2017).

?FaNeu2007? [15] R. FARWIG AND J. NEustupa, On the spectrum of a Stokes-type operator arising from flow around a rotating body, Manuscripta Math. 122 (2007), 419-437.

Faneu2009 [16] R. FARWig and J. Neustupa, On the spectrum of an Oseen-type operator arising from flow around a rotating body, Int. Equ. Oper. Theory 62 (2008), 169-189.

Faneu2010 [17] R. FARWig and J. Neustupa, Spectral properties in $L^{q}$ of an Oseen operator modelling fluid flow past a rotating body, Tohoku Math. J. 62 (2010), 287-309.

Fm [18] D. Fujiwara and H. Morimoto, An $L_{r}$-theorem of the Helmholtz decomposition of vector fields, J. Fac. Sci. Univ. Tokyo (1A) 24 (1977), 685-700.

FJK [19] S. FuČÍK, O. John And A. Kufner, Function Spaces, Noordhoff, Leyden, 1977.

GaRi [20] G. P. Galdi And S. Rionero, Weighted Energy Methods in Fluid Dynamics and Elasticity, Lecture Notes in Mathematics 1134, Springer, Berlin e.a., 1985.

GaPa [21] G. P. Galdi And M. Padula, A new approach to energy theory in the stability of fluid motion, Arch. Rat. Mech. Anal. 110 (1990), 187-286.

GHS [22] G. P. Galdi, J. G. Heywood and Y. Shibata, On the global existence and convergence to steady state of Navier-Stokes flow past an obstacle that is started from rest, Arch. Rat. Mech. Anal. 138 (1997), 307-318.

Ga1 [23] G. P. GALDI, An Introduction to the Mathematical Theory of the Navier-Stokes Equations, Vol. I: Linear Steady Problems, Springer Tracts in Natural Philosophy 38, Springer-Verlag, New York e.a., 1998.

Galdineu [24] G. P. GALDI, An Introduction to the Mathematical Theory of the Navier-Stokes Equations. Steady-State Problems, 2nd edition, Springer, New York e.a., 2011.

Gi [25] Y. GigA, Analyticity of the semigroup generated by the Stokes operator in $L^{r}$ spaces, Math. Z. 178 (1981), 297-329.

Henry [26] D. Henry, Geometric Theory of Semilinear Parabolic Equations, Lecture Notes in Mathematics 840, Springer, 1981.

Hey1 [27] J. G. HeYwood, On stationary solutions of the Navier-Stokes equations as limits of nonstationary solutions, Arch. Rat. Mech. Anal. 37 (1970), 48-60. 
Hey2 [28] J. G. Heywood, The exterior nonstationary problem for the Navier-Stokes equations, Acta Math. 129 (1972), 11-34.

Hey3 [29] J. G. HeYwood, The Navier-Stokes equations: On the existence, regularity and decay of solutions, Indiana Univ. Math. J. 29 (1980), 639-681.

Ki1 [30] H. KielhÖFER, Existenz und Regularität von Lösungen semilinearer parabolischer Anfangs-Randwertprobleme, Math. Z. 142 (1975), 131-160.

Ki2 [31] H. KIELhÖFER, On the Lyapunov stability of stationary solutions of semilinear parabolic differential equations, J. of Diff. Equations 22 (1976), 193-208.

KS [32] T. Kobayashi and Y. Shibata, On the Oseen equation in the three dimensional exterior domains, Math. Ann. 310 (1998), 1-45.

KSo [33] H. Kozono And H. Sohr, New a priori estimates for the Stokes equations in exterior domains, Indiana Univ. Math. J. 40 (1991), 1-27.

KoOg [34] H. Kozono And T. OGawa, On stability of Navier-Stokes flows in exterior domains, Arch. Rat. Mech. Anal. 128 (1994), 1-31.

KY2 [35] H. KozOnO AND M. YAmazAKI, On stability of small stationary solutions in Morrey spaces of the Navier-Stokes equations, Indiana Univ. Math. J. 44 (1995), 1307-1336.

KY3 [36] H. Kozono And M. YAmazaki, On a large class of stable solutions to the NavierStokes equations in exterior domains, Math. Z. 228 (1998), 751-785.

Mar [37] P. Maremonti, Asymptotic stability theorems for viscous fluid motions in exterior domains, Rend. Sem. Mat. Univ. Padova 71 (1984), 35-72.

Mas [38] K. Masuda, On the stability of incompressible viscous fluid motions past objects, J. Math. Soc. Japan 27 (1975), 294-327.

Mi2 [39] T. MiYaKAWA, On $L^{1}$-stability of stationary Navier-Stokes flows in $\mathbb{R}^{3}$, J. Math. Sci. Univ. Tokyo 4 (1997), 67-119.

Neri [40] U. NerI, Singular integrals, Lecture Notes in Mathematics 200, Springer, Berlin e.a., 1971.

Neu1 [41] J. NeustupA, Stabilizing influence of a skew-symmetric operator in semilinear parabolic equations, Rend. Mat. Sem. Univ. Padova 102 (1999), 1-18.

Neu1a [42] J. NeustupA, Selected topics in the theory of stability, CTU Reports 7 (2/1999), Czech Technical University in Prague.

Neu2 [43] J. NeustupA, Stability of a steady solution of a semilinear parabolic system in an exterior domain, Far East J. of Appl. Math. 15 (2004), 307-324.

Neu2010 [44] J. NeustupA, Stability of a steady viscous incompressible flow past an obstacle, J. Math. Fluid Mech. 11 (2009), 22-45. 
Neu2015 [45] J. NeustupA, A spectral criterion for stability of a steady viscous incompressible flow past an obstacle, to appear in J. Math. Fluid Mech.

Sa [46] D. H. SATtinger, The mathematical problem of hydrodynamic stability, J. of Math. and Mech. 18 (1970), 797-817.

Saz [47] L. I. SAzonov, Justification of the linearization method in the flow problem, Izv. Ross. Akad. Nauk Ser. Mat. 58 (1994), 85-109 (Russian).

Shi [48] Y. Shibata, On an exterior initial boundary value problem for Navier-Stokes equations, Quarterly of Applied Mathematics 57 (1999), 117-155.

SiSo [49] C. G. Simader AND H. Sohr, The Dirichlet problem for the Laplacian in bounded and unbounded domains, Pitman Resarch Notes in Mathematics 360, Addison Wesley Longman, Harlow, 1996.

Sohr [50] H. SoHR, The Navier-Stokes equations. An elementary functional analytic approach, Birkhäuser advanced texts, Basel-Boston-Berlin, 2001. 